Article

\title{
Long Time Behavior and Global Dynamics of Simplified Von Karman Plate Without Rotational Inertia Driven by White Noise
}

\author{
Huatao Chen ${ }^{1, *}$, Dengqing Cao ${ }^{1, *}$, Jingfei Jiang ${ }^{2}$ and Xiaoming Fan ${ }^{3}$ \\ 1 Division of Dynamics and Control, School of Astronautics, Harbin Institute of Technology, \\ Harbin 150001, China \\ 2 Division of Dynamics and Control, School of Mathematics and Statistics, \\ Shandong University of Technology, Zibo 255000, China; jjfrun@sdut.edu.cn \\ 3 School of Mathematics, Southwest Jiaotong University, Chengdu 610031, China; fanxm@swjtu.edu.cn \\ * Correspondence: htchencn@aliyun.com (H.C.); dqcao@hit.edu.cn (D.C.)
}

Received: 5 June 2018; Accepted: 22 July 2018; Published: 1 August 2018

check for updates

\begin{abstract}
Without the assumption that the coefficient of weak damping is large enough, the existence of the global random attractors for simplified Von Karman plate without rotational inertia driven by either additive white noise or multiplicative white noise are proved. Instead of the classical splitting method, the techniques to verify the asymptotic compactness rely on stabilization estimation of the system. Furthermore, a clear relationship between in-plane components of the external force that act on the edge of the plate and the expectation of radius of the global random attractors can be obtained from the theoretical results. Based on the relationship between global random attractor and random probability invariant measure, the global dynamics of the plates are analyzed numerically. With increasing the in-plane components of the external force that act on the edge of the plate, global $\mathcal{D}$-bifurcation, secondary global $\mathcal{D}$-bifurcation and complex local dynamical behavior occur in motion of the system. Moreover, increasing the intensity of white noise leads to the dynamical behavior becoming simple. The results on global dynamics reveal that random snap-through which seems to be a complex dynamics intuitively is essentially a simple dynamical behavior.
\end{abstract}

Keywords: Simplified clamped Von Karman plate without rotational inertia; global random attractors; global dynamics; random invariant measure; white noise

\section{Introduction}

\subsection{Background and Literatures Review}

There exists an essential difference between full Von Karman plate without rotational inertia and simplified Von Karman plate without rotational inertia. From the view of physics, the former takes account into the acceleration in-plane and the latter neglects it [1,2]. In the mathematical standpoint, the governing equations of full Von Karman plates without rotational inertia comprise coupled plate equations and wave equations, while the coupled plate equation and elliptic equation compose the governing equations of Von Karman plate [3].

The definition of global random attractors for random dynamical system (RDS) established by Arnold [4] were proposed by Crauel and Flandoli [5] and Schmalfuss [6]. The former developed the theory of global random attractors in phase space, while the random attractor is seen as a subset in the space of probability measures by Schmalfuss. Afterwards, Crauel et al. [7] introduced a notion of global random attractors which is accessible to the researcher who are not familiar to the probabilistic language. Furthermore, the assertion that global random attractors are uniquely determined by 
attracting deterministic compact sets in phase space was attained by Crauel in [8]. Invoking these theories, the existence of global random attractors for RDS related to a plenty of mathematical physics problems have been studied by many researchers, (e.g., [9-14] and the references therein).

Von Karman plate equation is a well-known model that arise in nonlinear elastodynamics, which can be found in many engineering applications, for instance, wing skin in airplane, vertical fins of High-speed aircraft, etc. For more details, see the Introduction in Monograph [1]. There is an abundant achievements on Von Karman plate and the brief list given below is by no means exhaustive. Invoking the adjoint method, Pappalardo and Guida [15] considered the optimal control problem associated with the vibration of Von Karman plates. Utilizing the theory of plate theory, monohull ship was modelled by Fortuna and Muscato [16]; moreover, the problem of identification and adaptive control were also investigated. To compute the modal parameters of the plates, the system identification algorithm was proposed by Pappalardo and Guida [17]. A survey on computational methods for motion of multibody systems (include plates) was made by Pappalardo and Guida [18]. For the sake of computing the motion of large deformation of the plates, Pappalardo et al. [19-21] developed different kinds of plate/shell finite elements.

From the mathematical view, to address the long time behavior of the mathematical physics problems, it must be verified that they can generate a dynamical system, which can be accomplished by achieving the existence and uniqueness of the solution for the systems. Lasiecka [22] was concerned with weak, classical and intermediate solutions to full von Karman equations. With respect to the nonautonomous case, one can refer to Leiva and Sivoli [23] and Abels et al. [24]. According to the proof of a "sharp regularity" estimates of the Von Karman bracket, the consequence of global existence, uniqueness and regularity of solutions for simplified Von Karman plate with nonlinear boundary dissipation can be founded in Favini et al. [25]. For more detail, one can refer to the Monograph [26]. As for the long time behavior of the Von Karman plate equations, the global attractors as well as inertial manifolds for the system in autonomous situation were studied by Chueshov and Lasiecka [27] and Chueshov and Lasiecka [28], respectively. Lasiecka [29] studied the uniform decay rates for thermoelastic full von karman system. Dynamics of a thermoelastic von Karman plate in a subsonic gas flow was addressed by Ryzhkova [30]. For a von Karman plate equation with a boundary memory condition which can even be a fractional damping, Park and Sun [31] tackled the uniform decay of the solution. For more detail of the research status of long time behavior of the Von Karman plate before the year 2010, one can refer to Monograph [26]. The study of long-time dynamics of a von Karman equation with time delay is due to Park [32]. Chueshov [33] investigated questions related to global attractors for delayed, nonrotational von Karman plates without any damping in the status of flow-structure interactions. Eliminating flutter for clamped von Karman plates in subsonic flows was concerned by Lasiecka and Webster [34]. Without assuming large values for the coefficient of damping, Khanmamedov [35] proved the existence of the global random attractors for Von Karman plate equation. With a very strict assumption on the coefficient of the weakly damping, the existence of global random attractors for simplified Von Karman plate without rotational inertia driven by multiplicative white noise was studied by Chen et al. [36].

The study on dynamics of Von Karman plates can be divided into two parts: the investigation of local dynamics and investigation of global dynamics. There exists abundant studies on local dynamics for Von Karman plates. For instance, invoking the Bubnov-Galerkin approach, Awrejcewicz and Krysko [37] analyzed the complex parametric vibrations of plates and shells. Nonlinear vibration and dynamic response as well as Thermal post-buckling of functionally graded thermoelastic Von Karman plates was considered by Huang and Shen [38] and Park and Kim [39], respectively. Employing the Homotopy perturbation technique, Rashidi et al. [40] studied the nonlinear vibration of Von Karman rectangular plate. Ghayesh et al. [41] tackled the nonlinear dynamics of axially moving Von Karmam plates. Ghayesh and Farokhi [42] were devoted to handling the nonlinear dynamics of Von Karman plate in MEMS. The nonlinear vibrations of viscoelastic Von Karman plate was analyzed by Amabili [43]. The global dynamics of nonlinear systems which can reveal more 
dynamic information than local dynamics are important in engineering applications. Compared with the literature on local dynamics, the investigation related to global dynamics is insufficient. Global dynamics of four-dimensional perturbed Hamiltonian systems and parametrically forced mechanical systems were addressed by Wiggins [44] and Feng and Wiggins [45], respectively. The technique employed in those works is Melnikov method which was invoked by Zhang to tackle a parametrically Von Karman plate in [46]. Due to the lack of analytical tools, numerical method is the main approach to study the global dynamics of nonlinear systems. According to Cell to Cell mapping method proposed by Hsu [47], Xu et al. [48] addressed global stochastic bifurcation in Duffing system.

There exist two standpoints in study on dynamics of random dynamical system, which are equivalent in the deterministic case, the "static" standpoint and the "dynamical" standpoint. However, two views are very different in the stochastic status (see $[4,49,50])$. The investigation on dynamics of the RDS associated with vibration of Von Karman plates in this paper means study the of "dynamical" dynamics of the systems; alternatively, the global dynamics in this paper are understood as the change in the pattern of existing probability invariant measures of the RDS. There exist some results on global dynamics on RDS. Crauel and Flandoli [49] asserted that additive noise destroys pitchfork bifurcation in one dimensional system. The statement that parametric noise (even a multiplicative white noise) destroys Hopf bifurcation was duo to Arnold et al. [51]. Wang [52] focused on the bifurcation for stochastic parabolic equations. The investigation on stochastic bifurcation in Duffing system by the theory of random attractors was due to Schenk-Hoppé [53]. According to some invariant manifolds to derive the lower bounds on the dimension of global random attractors, Caraballo et al. [54] studied the stochastic pitchfork bifurcation of the reaction diffusion equation with multiplicative white noise.

\subsection{Formulation and Contribution of This Investigation}

In some circumstances, the Von Karman plate equation that epitomizes certain distinct features and mathematical difficulties which lead to the "splitting method" [55], a traditional approach in the study on the existence of global random attractors, for extensive mathematical problems becomes invalid, such as SAVKP and SMVKP (introduced in Section 2.1). The existence of global attractors for the system in deterministic case (such as Chueshov and Lasiecka [27]) and stochastic case (e.g., [36]) relies on large enough value of damping coefficient. To our best knowledge, there hardly exists results on global random attractors for SAVKP and SMVKP with arbitrary small coefficient of the weakly damping.

Recently, the study on dynamics of Von Karman plate equation mainly focused on the local dynamics, inspired by Crauel [49] and Schenk-Hoppé [53]. Based on the existence of global random attractors and the relationship between invariant measure and global random attractor summarized in Proposition 2 in Section 3.1, the dynamics of Von Karman plate can be accomplished by employing the stochastic subdivision algorithm method proposed by Keller and Ochs [56] to achieve the global random attractors numerically.

As far as we know, the consequence of investigation in this aspect also do not be published in any composition. The purpose of this paper is to investigate the existence of global random attractors for SAVKP and SMVKP and to derive the global dynamics by achieving the structure of their global random attractors.

\subsection{Organization of the Paper}

The rest of this paper is organized as follows. In Section 2, the mathematical description of model and main results main results are given. Section 3 is intended to provided preliminary results employed in accomplishing the main proof which are given in Section 4. Finally, based on the main results listed in Section 3, summary and conclusions is made in Section 5.

Finally, to express the results and their respective proofs succinctly, the following conventions are made. Unless otherwise stated, in the sequel, the letter $C, \widetilde{C}, \widehat{C}, C_{i}, \widetilde{C}_{i}, \widehat{C}_{i}(i=1,2,3, \cdots$,$) are$ 
positive constants; in addition, $C(a, \cdots, b), \widetilde{C}(a, \cdots, b), \widehat{C}(a, \cdots, b)$ and $C_{i}(a, \cdots, b), \widetilde{C}_{i}(a, \cdots, b)$, $\widehat{C}_{i}(a, \cdots, b)(i=1,2,3, \cdots$,$) are positive constants depended on a, \cdots, b$.

\section{The Mathematical Model and Main Results}

Section 2.1 is used to make the mathematical description of the model considered in this paper. The main results of this paper are listed in Section 2.2.

\subsection{Mathematical Description of the Model}

Let $D \subset \mathbb{R}^{2}$ be a bounded domain with boundary $\Gamma$; without loss of generality, assume the origin 0 belongs to $\Gamma$. Suppose $(x, y) \in \Gamma$ is an arbitrarily given point, while $\Gamma(x, y)$ denotes the arc, oriented in the usual manner, joining the origin 0 to the point $(x, y)$ along the boundary of $D$. For more details, one can refer to Ciarlet [3]. The governing equation of simplified Von Karman plate without rotational inertia is:

$$
\rho h U_{t t}+\operatorname{ch} G\left(U_{t}\right)+\bar{D} \Delta^{2} U=P+[V(U), U]
$$

with the clamped boundary

$$
x \in \partial D: U=\frac{\partial U}{\partial n}=0,
$$

where $U$ is transversal displacement of the plate. $[\cdot, \cdot]$ is Von Karman bracket [26] (also known as Monge-Ampère form[3]) with the form of

$$
[v, u]=\frac{\partial^{2} u}{\partial x_{1}^{2}} \frac{\partial^{2} v}{\partial x_{2}^{2}}+\frac{\partial^{2} v}{\partial x_{1}^{2}} \frac{\partial^{2} u}{\partial x_{2}^{2}}-2 \frac{\partial^{2} u}{\partial x_{1} \partial x_{2}} \frac{\partial^{2} v}{\partial x_{1} \partial x_{2}} .
$$

$V(U)$ is the Airy function satisfies

$$
\begin{aligned}
& \Delta^{2} V=-\frac{E h}{2}[U, U], \\
& x \in \Gamma: V=\phi_{0}, \frac{\partial V}{\partial n}=\phi_{1},
\end{aligned}
$$

in which the physical parameter $E, \rho, h, I, D$ can refer to [1]. $\phi_{0}, \phi_{2}$ is defined as

$$
\phi_{0}(x, y)=-x \int_{\Gamma(x, y)} f_{2} d s+y \int_{\Gamma(x, y)} f_{1} d s+\int_{\Gamma(x, y)} \bar{x} f_{2}-\bar{y} f_{1} d s,
$$

and

$$
\phi_{1}(x, y)=-n_{1}(x, y) \int_{\Gamma(x, y)} f_{2} d s+n_{2}(x, y) \int_{\Gamma(x, y)} f_{1} d s
$$

where $f_{1}, f_{2}$ are components of the in-plane force on boundary along the direction $x, y$, which comply with

$$
n_{1} N_{11}+n_{2} N_{12}=f_{1}, n_{1} N_{12}+n_{2} N_{22}=f_{2}
$$

where $N_{11}, N_{12}, N_{22}$ are membrane forces in the plate; for more details, one can refer to [3]. Moreover, let $V=V_{0}+V_{1}, V_{0}$ be the solution of the following system

$$
\begin{aligned}
& \Delta^{2} V_{0}=-\frac{E h}{2}[w, w], \\
& V_{0}=\frac{\partial V_{0}}{\partial n}=0 .
\end{aligned}
$$


$V_{1}$ satisfies

$$
\begin{aligned}
& \Delta^{2} V_{1}=0, \\
& V_{1}=\phi_{0}, \frac{\partial V_{1}}{\partial n}=\phi_{1} .
\end{aligned}
$$

Thus, Equation (1) can be rewritten as:

$$
\rho h U_{t t}-\rho I \Delta U_{t t}+\operatorname{ch} G\left(U_{t}\right)+\bar{D} \Delta^{2} U=F+P+\left[V_{1}+V_{0}, U\right] .
$$

In some cases, only $V_{0}$ is named Airy function, while $V_{1}$ is called the in-plane force, (see Chueshov and Lasiecka [26]). This convention is employed in this paper.

To formulate the system tackled in this paper, some spaces are introduced in the following. Let $\|u\| \equiv\|u\|_{L^{2}(D)},\|u\|_{s} \equiv\|u\|_{H_{0}^{s}(D)},(u, v) \equiv(u, v)_{L^{2}(D)},(u, v)_{s}=(u, v)_{H_{0}^{s}(D)}$, where $H^{s}(D), H_{0}^{s}(D)$, $s \in \mathbb{R}$ are the usual Sobolev Spaces. Let $A=\Delta^{2}: H_{0}^{2} \cap H^{4}(D) \rightarrow L^{2}(D)$, then $A$ is self-adjoint, positive, unbounded linear operators and $A^{-1} \in \mathscr{L}\left(L^{2}(D)\right)$ is compact. Therefore, their eigenvalues $\left\{\Lambda_{i}\right\}_{i \in \mathbb{N}}$ satisfy $0<\Lambda_{1} \leq \Lambda_{2} \leq \cdots \rightarrow \infty$ and the corresponding eigenvalues $\left\{w_{i}\right\}_{i=1}^{\infty}$ forms an orthonormal basis in $L^{2}(D)$. Then, we can interpret the power of $A^{s}, s \in \mathbb{R}$ by the method developed by Temam [55]. Specifically, $\left\|A^{\frac{1}{4}} u\right\|=\|\nabla u\|,\left\|A^{\frac{1}{2}} u\right\|=\|\Delta u\|, \forall u \in \mathcal{D}(A)$, however, it is mentioned here that $A^{\frac{1}{2}} \neq$ $-\Delta, A^{\frac{1}{4}} \neq \nabla$. Nevertheless, it is emphasized here that $A^{\frac{1}{2}} \neq-\Delta$ with the boundary in Equation (2). In fact, the operator $A_{1}:=-\Delta$ with the boundary condition in Equation (2) is a self-adjoint, positive, unbounded linear operators from $L^{2}(D)$ to $L^{2}(D)$ and $A^{-1} \in \mathscr{L}\left(L^{2}(D), L^{2}(D)\right)$ is compact. Thus, the power of $(-\Delta)^{s}, s \in \mathbb{R}$ can be defined; furthermore, $\left\|A_{1}^{s} u\right\| \leq\left\|A^{\frac{s}{2}} u\right\|, \forall s \in[0,1], u \in \mathcal{D}(A)$.

Suppose $P$ is a stochastic pressure signified by white noise, then the dynamics equation of abstract dimensionless clamped simplified Von Karman plate without rotational inertia driven by white noise are as follow

$$
\begin{gathered}
u_{t t}+\alpha u_{t}+A u-k\left[\mathcal{F}(u)+F_{0}, u\right]=\sigma q(x) \dot{W}, \\
u_{t t}+\alpha u_{t}+A u-k\left[\mathcal{F}(u)+F_{0}, u\right]=\sigma u \dot{W}
\end{gathered}
$$

with the clamped boundary condition

$$
x \in \partial D: u=\frac{\partial u}{\partial n}=0,
$$

where $u: \mathbb{R} \times D \rightarrow \mathbb{R}$ is the dimensionless transversal displacement of the plate. $\sigma \geq 0, k>0$ are a given constant,

$$
q(x) \in\left(H_{0}^{2} \cap H^{4}\right)(D) .
$$

$W$ is the one dimensional two-sided real-valued standard Wiener process, and $\dot{W}$ is called white noise. $\alpha>0$ is the coefficient of damping.

Equation (10a) describe abstract dimensionless clamped simplified Von Karman plate without rotational inertia driven by additive/multiplicative white noise, respectively. Furthermore, $\mathcal{F}$ satisfies

$$
\begin{aligned}
& \Delta^{2} \mathcal{F}=-[u, u], \\
& x \in \Gamma: \mathcal{F}=\frac{\partial \mathcal{F}}{\partial n}=0
\end{aligned}
$$

and $F_{0}$ is in agreement with 


$$
\begin{aligned}
& \Delta^{2} F_{0}=0, \\
& x \in \Gamma: F_{0}=g_{1}, \frac{\partial F_{0}}{\partial n}=g_{2}
\end{aligned}
$$

where $g_{1} \in H^{\frac{3}{2}}(\Gamma), g_{2} \in H^{\frac{1}{2}}(\Gamma)$ are derived from Equations (3) and (4). By the monograph of Lions and Magenes [57], $F_{0} \in H^{2}(D)$, define

$$
N(u)=k\left[\mathcal{F}(u)+F_{0}, u\right] .
$$

The system described by Equations (10a), (10c), and (10e)-(10h) is denoted by SAVKP. The system interpreted by Equations (10b), (10c), and (10e)-(10h) is represented by SMVKP.

Invoking the compactness of $A$, the Hilbert space $\mathcal{D}\left(A^{s}\right), s \in \mathbb{R}$ with norm $\|\cdot\|_{s}$ and $(\cdot, \cdot)_{s}$ can be defined as the mechanism in Temam [55], especially, $\mathcal{D}\left(A^{\frac{1}{2}}\right)=H_{0}^{2}(D)$. Moreover, for all $s_{1}, s_{2} \in \mathbb{R}, s_{1} \geq s_{2}, \mathcal{D}\left(A^{s_{1}}\right)$ can be compact imbedding in $\mathcal{D}\left(A^{s_{2}}\right)$ and the following holds

$$
\|u\|_{s_{1}} \geq \Lambda_{1}^{\frac{s_{1}-s_{2}}{2}}\|u\|_{s_{2}}, \forall u \in \mathcal{D}\left(A^{s_{1}}\right) .
$$

Let $E_{1+2 s}=\mathcal{D}\left(A^{\frac{1}{2}+s}\right) \times \mathcal{D}\left(A^{s}\right), 0 \leq s \leq \frac{1}{2}$ equipped with Graph norm and the induced inner product, then they are all Hilbert spaces.

Let $\left(X,\|\cdot\|_{X}\right)$ be a separable space with Borel $\sigma-$ algebra $\mathcal{B}(X)$ and $(\Omega, \mathcal{F}, P)$ be a probability space. $\left\{\theta_{t}: \Omega \rightarrow \Omega, t \in \mathbb{R}\right\}$ is a family of measure preserving transformations such that $(t, \omega) \rightarrow \theta_{t} \omega$ is measurable, $\theta_{0}=\mathrm{id}, \theta_{t+s}=\theta_{t} \circ \theta_{s}$ for all $s, t \in \mathbb{R}$. Then, the flow $\theta_{t}$ together with the probability space $\left(\Omega, \overline{\mathcal{F}}, P,\left\{\theta_{t}\right\}_{t \in \mathbb{R}}\right)$ is called a metric dynamical system. For the particular applications in this paper, the metric dynamical systems generated by a one dimensional two-sided standard Wiener process defined on a Probability space $(\Omega, \overline{\mathcal{F}}, \mathbb{P})$ is introduced there. Let $\Omega=\{\omega \mid \omega(\cdot) \in \mathbb{C}(\mathbb{R}, \mathbb{R}), \omega(0)=0\}$, $\overline{\mathcal{F}}$ is the $\sigma$-algebra induced by the compact open topology for this set and $\mathbb{P}$ is the Wiener measure on $\mathcal{F}$. Set

$$
\theta_{t} \omega(\cdot)=\omega(\cdot+t)-\omega(\cdot), \forall t \in \mathbb{R},
$$

according to Arnold [4], we have $\mathbb{P}$ is ergodic with respect to the flow $\left\{\theta_{t}\right\}_{t \in \mathbb{R}}$. Thus, $\left(\Omega, \overline{\mathcal{F}}, \mathbb{P},\{\theta\}_{t \in \mathbb{R}}\right)$ is the metric dynamical systems employed in this paper. Moreover, the Ornstein-Uhlenbeck process, which should be used in transforming a stochastic system to a random system, is introduced as follows

$$
\left\{\begin{array}{l}
d z+\mu z d t=d W \\
z(-\infty)=0
\end{array}\right.
$$

in which $\mu>0$. The general form for the solution of Equation (13) is

$$
z(t)=\int_{-\infty}^{t} e^{-\mu(t-s)} d W
$$

Let

$$
z\left(\theta_{t} \omega\right):=-\mu \int_{-\infty}^{0} e^{\mu \tau}\left(\theta_{t} \omega\right)(\tau) d \tau
$$

where $\left\{\theta_{t}\right\}_{t \in \mathbb{R}}$ is defined by Equation (12) in Section 2. Merging with integration by parts, $z\left(\theta_{t} \omega\right)$ is the solution for the system in Equation (13).

Although by no means always, it will be convenient to reduce Equations (10a) and (10b) to an 
evolution equation of the first order in time in the following manner. Let $\underline{v}=u_{t}+\varepsilon u, \mathbf{U}=\left[U_{1}, U_{2}\right]^{T}=$ $[u, \underline{v}]^{T}$, then Equation (10a) can be transformed to the ensuing form

$$
\frac{d \mathbf{U}}{d t}=\mathbf{A}_{1} \mathbf{U}+\mathbf{F}_{1}(\mathbf{U})
$$

where

$$
\mathbf{A}_{1}=\left(\begin{array}{cc}
-\varepsilon I, & I \\
-A+\varepsilon(\alpha-\varepsilon), & -(\alpha-\varepsilon) I
\end{array}\right), \quad \mathbf{F}_{1}(\mathbf{U})=\left(\begin{array}{c}
\mathbf{F}_{11}(\mathbf{U}) \\
\mathbf{F}_{12}(\mathbf{U})
\end{array}\right)
$$

and

$$
\mathbf{F}_{11}(\mathbf{U})=0, \mathbf{F}_{12}=N\left(U_{1}\right)+\sigma q(x) \dot{W} .
$$

The system described by Equations (10c), (15) and (10e)-(10h) denoted by SAVKPT1. Obviously, SAVKP is equivalent to SAVKPT1.

To accomplish the stabilization estimation for the solution of SAVKPT1, the following systems is needed. Suppose $\mathbf{U}^{(1)}=\left[U_{1}^{(1)}, U_{2}^{(1)}\right]=\left[u_{1}, \underline{v}_{1}\right], \mathbf{U}^{(2)}=\left[U_{1}^{(2)}, U_{2}^{(2)}\right]=\left[u_{2}, \underline{v}_{2}\right]$ are two solution of SAVKPT1, then

$$
\frac{d \mathbf{U}^{(1)}-\mathbf{U}^{(2)}}{d t}=\mathbf{A}_{1}\left(\mathbf{U}^{(1)}-\mathbf{U}^{(2)}\right)+\widetilde{\mathbf{F}}_{1}\left(\mathbf{U}^{(1)}-\mathbf{U}^{(2)}\right),
$$

where $\widetilde{\mathbf{F}}_{1}\left(\mathbf{U}^{(1)}-\mathbf{U}^{(2)}\right)=\mathbf{F}_{1}\left(\mathbf{U}^{(1)}\right)-\mathbf{F}_{1}\left(\mathbf{U}^{(2)}\right)$.

Furthermore, let $v=\underline{v}-\sigma q(x) z\left(\theta_{t} \omega\right), \boldsymbol{\Phi}=[u, v]^{T}$ and

$$
[u, v]^{T}=\left[u, \underline{v}-\sigma q(x) z\left(\theta_{t} \omega\right)\right]^{T} \equiv T^{A}\left(\theta_{t} \omega\right)[u, \underline{v}]^{T}, \forall[u, \underline{v}] \in E_{1},
$$

thus

$$
\frac{d \boldsymbol{\Phi}}{d t}=\mathbf{A}_{1} \boldsymbol{\Phi} d t+\mathbf{F}_{2}\left(z\left(\theta_{t} \omega\right)\right)+\mathbf{F}_{3}(\boldsymbol{\Phi})
$$

where $\boldsymbol{\Phi}=[u, v]^{T}$,

$$
\mathbf{F}_{2}\left(z\left(\theta_{t} \omega\right)\right)=\left(\begin{array}{c}
F_{21}\left(z\left(\theta_{t} \omega\right)\right) \\
F_{22}\left(z\left(\theta_{t} \omega\right)\right)
\end{array}\right)=\left(\begin{array}{c}
\sigma q(x) z\left(\theta_{t} \omega\right) \\
\sigma(\mu-\alpha+\varepsilon) q(x) z\left(\theta_{t} \omega\right)
\end{array}\right), \quad \mathbf{F}_{3}(\mathbf{\Phi})=\left(\begin{array}{c}
F_{31}(\boldsymbol{\Phi}) \\
F_{32}(\boldsymbol{\Phi})
\end{array}\right)
$$

here

$$
F_{31}(\boldsymbol{\Phi})=0, F_{32}(\mathbf{\Phi})=N\left(U_{1}\right)
$$

Equation (18) is a partial differential equations with random coefficient which can be studied $\omega$ by $\omega$. Let SAVKPT2 signify the system described by Equations (18), (10c) and (10e)-(10h). It is emphasized that SAVKPT2 is not equivalent to SAVKPT1.

Analogously, let $\underline{v}=u_{t}+\varepsilon u, \mathbf{U}=\left[U_{1}, U_{2}\right]^{T}=[u, \underline{v}]^{T}$ and

$$
[u, v]^{T}=\left[u, \underline{v}-\sigma u z\left(\theta_{t} \omega\right)\right]^{T} \equiv T^{M}\left(\theta_{t} \omega\right)[u, \underline{v}]^{T}, \forall[u, v] \in E_{1}, \omega \in \Omega,
$$

define $\boldsymbol{\Phi}=\left[\Phi_{1}, \Phi_{2}\right]^{T}=[u, v]^{T}$, the following system associated with SMVKP can be attained

$$
\frac{d \mathbf{U}}{d t}=\mathbf{A}_{1} \mathbf{U}+\mathbf{F}_{1}(\mathbf{U})
$$

in which

$$
\mathbf{A}_{1}=\left(\begin{array}{cc}
-\varepsilon I, & I \\
-A+\varepsilon(\alpha-\varepsilon), & -(\alpha-\varepsilon) I
\end{array}\right), \quad \mathbf{F}_{1}=\left(\begin{array}{c}
F_{11}(\mathbf{U}) \\
F_{12}(\mathbf{U})
\end{array}\right)
$$


and

$$
\begin{gathered}
\mathbf{F}_{11}(\mathbf{U})=0, \mathbf{F}_{12}(\mathbf{U})=N\left(U_{1}\right)+\sigma U_{1} \dot{W} . \\
\frac{d \boldsymbol{\Phi}}{d t}=\mathbf{A}_{1} \boldsymbol{\Phi}+\mathbf{F}_{2}\left(\boldsymbol{\Phi}, \theta_{t} \omega\right)+\mathbf{F}_{3}\left(\boldsymbol{\Phi}, \theta_{t} \omega\right)
\end{gathered}
$$

where

$$
\begin{gathered}
\mathbf{F}_{2}\left(\boldsymbol{\Phi}, \theta_{t} \omega\right)=\left(\begin{array}{c}
F_{21}\left(\boldsymbol{\Phi}, \theta_{t} \omega\right) \\
F_{22}\left(\boldsymbol{\Phi}, \theta_{t} \omega\right) \\
F_{31}\left(\boldsymbol{\Phi}, \theta_{t} \omega\right) \\
F_{32}\left(\boldsymbol{\Phi}, \theta_{t} \omega\right)
\end{array}\right)=\left(\begin{array}{c}
\sigma z\left(\theta_{t} \omega\right) \Phi_{1} \\
-\sigma z\left(\theta_{t} \omega\right) \Phi_{2}
\end{array}\right), \\
\mathbf{F}_{3}\left(\boldsymbol{\Phi}, \theta_{t} \omega\right)=0, F_{32}\left(\boldsymbol{\Phi}, \theta_{t} \omega\right)=\sigma(\mu-\alpha+2 \varepsilon) z\left(\theta_{t} \omega\right) \Phi_{1}-\sigma^{2} z^{2}\left(\theta_{t} \omega\right) \Phi_{1}+N\left(\Phi_{1}\right),
\end{gathered}
$$

SMVKPT1 represents the systems defined by Equations (20), (10c) and (10e)-(10f), then SMVKP and SMVKPT1 are equivalent. The system described by Equations (21), (10c) and (10e)-(10f) is denoted by SMVKPT2.

Furthermore, assume $\boldsymbol{\Phi}^{(\mathbf{1})}=\left[\Phi_{1}^{(1)}, \Phi_{2}^{(1)}\right]^{T}=\left[u_{1}, v_{1}\right]^{T}, \boldsymbol{\Phi}^{(2)}=\left[\Phi_{1}^{(2)}, \Phi_{2}^{(2)}\right]^{T}=\left[u_{2}, v_{2}\right]^{T}$ are two solutions of SAVKPT2, thus

$$
\frac{d \boldsymbol{\Phi}^{(\mathbf{1})}-\boldsymbol{\Phi}^{(2)}}{d t}=\mathbf{A}_{1}\left(\boldsymbol{\Phi}^{(\mathbf{1})}-\boldsymbol{\Phi}^{(2)}\right)+\mathbf{D F}_{1}\left(\boldsymbol{\Phi}^{(\mathbf{1})}-\boldsymbol{\Phi}^{(2)}, \theta_{t} \omega\right)+\mathbf{D F}_{2}\left(\boldsymbol{\Phi}^{(\mathbf{1})}-\boldsymbol{\Phi}^{(2)}, \theta_{t} \omega\right),
$$

in which

$$
\mathbf{D F}_{1}\left(\boldsymbol{\Phi}^{(\mathbf{1})}-\boldsymbol{\Phi}^{(\mathbf{2})}, \theta_{t} \omega\right)=\left(\begin{array}{c}
D F_{11}\left(\boldsymbol{\Phi}^{(\mathbf{1})}-\boldsymbol{\Phi}^{(\mathbf{2})}, \theta_{t} \omega\right) \\
D F_{12}\left(\mathbf{\Phi}^{(\mathbf{1})}-\boldsymbol{\Phi}^{(\mathbf{2})}, \theta_{t} \omega\right) \\
D F_{21}\left(\boldsymbol{\Phi}^{(\mathbf{1})}-\boldsymbol{\Phi}^{(\mathbf{2})}, \theta_{t} \omega\right) \\
D F_{22}\left(\boldsymbol{\Phi}^{(\mathbf{1})}-\boldsymbol{\Phi}^{(2)}, \theta_{t} \omega\right)
\end{array}\right)=\mathbf{F}_{2}\left(\boldsymbol{\Phi}, \theta_{t} \omega\right)-\mathbf{F}_{2}\left(\boldsymbol{\Phi}, \theta_{t} \omega\right),
$$

Equation (22) is used to obtain the stabilization estimation for SMVKPT1.

Remark 1. For the sake of brevity, when no ambiguity is possible, the symbols used in SAVKPT and SMVKPT, $S A V K P T 1$ and SMVKPT1, and SAVKPT2 and SMVKPT2 are the same or similar. Since each of the symbols has a clearl explanation, it is not confusing to express the main results in this paper. However, it must be kept in mind that they are not the same.

\subsection{Main Results}

Approved by the equivalent between SAVKPT and SAVKPT1, and SMVKPT and SMVKPT1, it is enough to only address the dynamical behavior of SAVKPT1 and SMVKPT1. This subsection is used to present the main results of this paper. Let

$$
\varepsilon_{0}=\frac{\Lambda_{1} \alpha}{2 \Lambda_{1}+\alpha^{2}}, 0<\varepsilon \leq \varepsilon_{0},
$$

\subsubsection{Random Attractors in Additive White Noise Case}

This part is devoted to providing the main results for SAVKPT1. The following Theorem considers the existence and uniqueness of solution for SAVKPT2.

Theorem 1. For any given initial value $t=\tau: \boldsymbol{\Phi}=\boldsymbol{\Phi}_{\tau} \in E_{1}$, there exists a uniqueness (mild) solution for $\operatorname{SAVKPT2} \boldsymbol{\Phi}(t, \tau, \omega) \in C\left([\tau, \tau+T], E_{1}\right), t \in[\tau, \tau+T], \forall T>0$. 
Furthermore, let $\boldsymbol{\Phi}^{A}\left(t, \tau, \omega ; \boldsymbol{\Phi}_{\tau}\right)=\varphi^{A}(t, \tau, \omega) \boldsymbol{\Phi}_{\tau}$, which means that $\varphi^{A}(t, \tau, \omega)$ is the solution mapping of SAVKPT2. Then,

$$
\varphi^{A}\left(t-\tau, 0, \theta_{\tau} \omega\right)=\varphi^{A}(t, \tau, \omega), \varphi^{A}(\tau, \tau, \omega)=i d
$$

Let

$$
S_{\varepsilon}^{A}(t, \omega)=\varphi^{A}(t, 0, \omega)=\varphi^{A}\left(0,-t, \theta_{t} \omega\right), \forall t \geq 0, \forall \omega \in \Omega,
$$

by means of Theorem $1, S_{\varepsilon}^{A}(t, \omega)$ is the RDS induced by SAVKPT2. Correspondingly, SAVKPT1 can also generate a $\operatorname{RDS} S^{A}(t, \omega)$ which is defined as

$$
S^{A}(t, \omega)=T^{A}\left(\theta_{t} \omega\right)^{-1} S_{\varepsilon}^{A}(t, \omega) T^{A}(\omega): E_{1} \rightarrow E_{1} .
$$

here $T^{A}\left(\theta_{t} \omega\right)$ is defined by Equation (17). Furthermore, the solution mapping determined by SAVKPT1 is denoted by $\phi^{A}(t, \tau, \omega)$, then

$$
S^{A}(t, \omega)=\phi^{A}(t, 0, \omega)=\phi^{A}\left(0,-t, \theta_{t} \omega\right), \forall t \geq 0, \forall \omega \in \Omega .
$$

The following turns to the existence of global random attractors for SAVKPT1.

Let $\delta_{0}$ be any given positive constant, $\bar{k}=\max \{1, k\}$ and

$$
\begin{aligned}
\mu & >\mu_{0}=\frac{64|\sigma|^{2}\|q\|^{2}}{\pi \varepsilon^{2}} \\
K & =K_{0}+\left.\left|z\left(\theta_{t} \omega\right) K_{1}+\right| z\left(\theta_{t} \omega\right)\right|^{2} K_{2} \\
\rho(\omega) & =\sigma^{2}\|q(x)\|^{2}|z(\omega)|^{2}+2 \int_{-\infty}^{0} e^{\epsilon t+\int_{t}^{0} 2 \sqrt{k}\left|\sigma z\left(\theta_{s} \omega\right)\right|\|q\| d k} K d s+\delta_{0}, \\
r(\omega) & =\sqrt{\rho(\omega)} .
\end{aligned}
$$

in which

$$
\begin{aligned}
& K_{0}=\frac{3 k \varepsilon}{2} C\left(\frac{7}{6}, C_{1}, C_{2}\right)\left\|F_{0}\right\|_{2}^{2}, K_{1}=2 \sqrt{\bar{k}} k|\sigma|\|q\| C\left(\frac{1}{4}, C_{1}, C_{2}\right)\left\|F_{0}\right\|_{2}^{2}, \\
& K_{2}=\frac{2 \sigma^{2}(\mu-\alpha+\varepsilon)^{2}}{k_{0}}\|q(x)\|^{2}+\frac{4 k^{2}|\sigma|^{2}\|q\|_{1}^{2}\left\|F_{0}\right\|_{2}^{2}}{\varepsilon}+\frac{4 \sigma^{2}}{\varepsilon}\left\|A^{\frac{1}{2}} q(x)\right\|^{2},
\end{aligned}
$$

$C\left(\eta, C_{1}, C_{2}\right), \eta=\frac{1}{4}, \frac{7}{6}$ are constants that satisfy Equation (45) in Section 3.2.1.

The next theorem considers the existence and expectation of radius of global random attractors for $S^{A}(t, \omega)$.

Theorem 2. $S^{A}(t, \omega)$ possesses the global random attractors $\mathscr{A}(\omega), \omega \in \Omega$ in $E_{1}$ which satisfies $\mathbb{P}$-a.s.

$$
\begin{aligned}
& \mathscr{A}(\omega) \subseteq \mathscr{B}(0, r(\omega)), \\
& \mathbb{E}(\rho(\omega)) \leq M<\infty .
\end{aligned}
$$

where $\mathscr{B}(0, r(\omega))$ denotes the open ball centered at the origin with radius is $r(\omega)$, while $M$ is given by Equation (A24) in Section 3.2.1.

\subsubsection{Random Attractors in Multiplicative White Noise Case}

The main results for SMVKPT1 is given in this part.

The form of following theorem is very similar to the Theorem 1.

Theorem 3. For any given initial value $t=\tau: \mathbf{\Phi}=\boldsymbol{\Phi}_{\tau} \in E_{1}$, SMVKPT2 possesses uniqueness (mild) solution $\boldsymbol{\Phi}^{M}\left(t, \tau, \omega ; \boldsymbol{\Phi}_{\tau}\right) \in C\left([\tau, \tau+T], E_{1}\right), t \in[\tau, \tau+T], T>0$. 
Furthermore, set $\boldsymbol{\Phi}^{M}\left(t, \tau, \omega ; \boldsymbol{\Phi}_{\tau}\right)=\varphi^{M}(t, \tau, \omega) \boldsymbol{\Phi}_{\tau}$, which indicates that $\varphi^{B}(t, \tau, \omega)$ is the solution mapping of SMVKPT2. Thus,

$$
\varphi^{M}\left(t-\tau, 0, \theta_{\tau} \omega\right)=\varphi^{M}(t, \tau, \omega), \varphi^{M}(\tau, \tau, \omega)=i d .
$$

Therefore, set

$$
S_{\varepsilon}^{M}(t, \omega)=\varphi^{M}(t, 0, \omega)=\varphi^{M}\left(0,-t, \theta_{t} \omega\right), \forall t \geq 0, \forall \omega \in \Omega,
$$

then, $S_{\varepsilon}^{M}(t, \omega)$ the RDS induced by SMVKPT2 and $\operatorname{RDS} S^{M}(t, \omega)$ defined as follow

$$
S^{M}(t, \omega)=T^{M}\left(\theta_{t} \omega\right)^{-1} S_{\varepsilon}^{M}(t, \omega) T^{M}(\omega): E_{1} \rightarrow E_{1} .
$$

is the RDS generate by SMVKPT1, where $T^{M}\left(\theta_{t} \omega\right)$ is defined by Equation (19). In additive, let

$$
S^{M}(t, \omega)=\phi^{M}(t, 0, \omega)=\phi^{M}\left(0,-t, \theta_{t} \omega\right), \forall t \geq 0, \forall \omega \in \Omega .
$$

Thus, $\phi(t, \tau, \omega)$ is the solution mapping of SMVKPT1.

The following turns to the existence of global random attractors for SMVKPT1.

Suppose $\delta_{0}$ is any given positive constant, $\mu=\alpha-2 \varepsilon$ and

$$
\frac{3 \sigma^{2}}{\mu \sqrt{\Lambda_{1}}}+\frac{12 \sigma}{\sqrt{\pi \mu}}<\varepsilon
$$

Set

$$
\begin{aligned}
C_{0}\left(\theta_{t} \omega\right) & =3\left(2\left|\sigma z\left(\theta_{t} \omega\right)\right|+\frac{\sigma^{2}\left|z\left(\theta_{t} \omega\right)\right|^{2}}{\sqrt{\Lambda_{1}}}\right), \rho_{0}(\omega)=\int_{-\infty}^{t} e^{\varepsilon t+\int_{t}^{0} C_{0}\left(\theta_{s} \omega\right) d s} K d s+\delta_{0} \\
\rho(\omega) & =\rho_{0}(\omega)+k C\left(\frac{1}{2}, C_{1}, C_{2}\right)\left\|F_{0}\right\|_{2}^{2}, K_{0}=\varepsilon k C\left(\frac{3}{2}, C_{1}, C_{2}\right)\left\|F_{0}\right\|_{2}^{2} \\
K_{1} & =8 k C\left(\frac{1}{8}, C_{1}, C_{2}\right)\left\|F_{0}\right\|_{2}^{2}, K_{2}=\frac{4 k \sigma^{2}}{\sqrt{\Lambda_{1}}} C\left(\frac{1}{8}, C_{1}, C_{2}\right)\left\|F_{0}\right\|_{2}^{2} \\
K & =K_{0}+\left|z\left(\theta_{t} \omega\right)\right| K_{1}+\left|z\left(\theta_{t} \omega\right)\right|^{2} K_{2} \\
r(\omega) & =\sqrt{\left(\frac{|\sigma z(\omega)|^{2}}{\Lambda_{1}}+1\right) \rho(\omega)}
\end{aligned}
$$

where $C\left(\eta, C_{1}, C_{2}\right), \eta=\frac{1}{2}, \frac{3}{2}, \frac{1}{8}$ are constants satisfying Equation (45) in Section 3.2.2. Then, the existence and finite expectation of radius of global random attractors for $S^{M}(t, \omega)$ can be asserted by the next theorem.

Theorem 4. There exists global random attractors $\mathscr{A}(\omega), \omega \in \Omega$ for $S^{M}(t, \omega)$ in $E_{1}$; moreover,

$$
\begin{aligned}
& \mathscr{A}(\omega) \subset \mathscr{B}(0, r(\omega)), \mathbb{P}-\text { a.s. } \\
& \mathbb{E}(\rho(\omega)) \leq M<\infty,
\end{aligned}
$$

where $\mathscr{B}(0, r(\omega))$ denotes the open ball centered at the origin with radius is $r(\omega)$, while $M$ is given by Equation (A43) in Section 3.2.2.

Comparing Theorems 1 and 3, as well as Theorems 2 and 4, their forms are the same or similar, while there exists essential difference between them, which is expounded in Section 5. 


\subsubsection{Global Dynamics in Both Additive and Multiplicative White Noise Cases}

Based on the theoretical results and the relationship between invariant measures and global attractors introduced in Proposition 2 in Section 3.1, the rest of this subsection is dedicated to studying the global dynamics of the stochastic Von Kaman plates, which is accomplished by deriving the components of global random attractor numerically, the main components are referred as global random point attractor and global random basic attractor. The modal equations associated with the stochastic Von Karman plates which are not display here (see Equation (A1) in Appendix A) can be obtained by employing inertial manifold with delay [58] and nonlinear gakerlin method [59].

Let $s_{1}=2, s_{2}=2, s_{0}=4, D=[0,8] \times[0,6] \subset \mathbb{R}^{2}$ in Appendix A, Figure 1 shows the model for vibration of Von Karman plate. The eigenvalues $\left\{\Lambda_{i}\right\}_{i=1}^{4}$ and eigenvectors $\left\{w_{i}\right\}_{i=1}^{4}$ of operator $A$ and integration with respect to space variable in Equation (A2) listed in Appendix A can be performed by COMSOL with Matlab [60], and then the solution of model equations can be obtained by stochastic Runge-Kutta method [61].

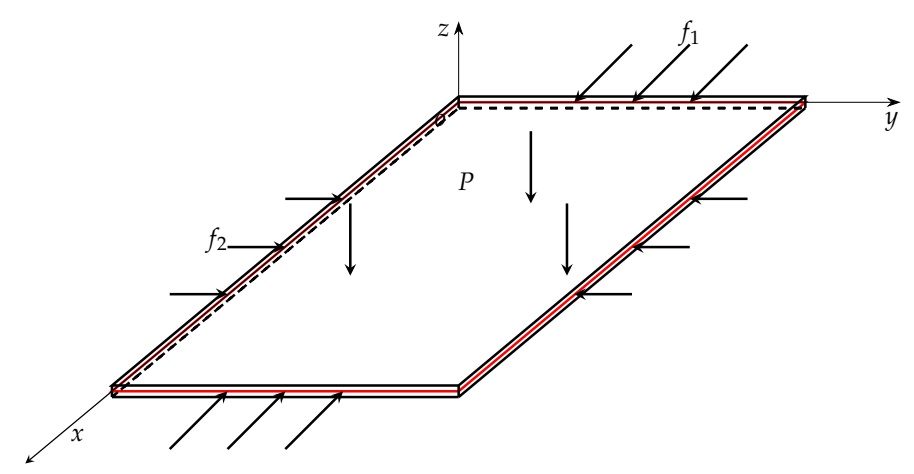

Figure 1. The model for vibration of Von Kaman plate under the random loading.

The situation of additive white noise. In this case, the $P$ in Figure 1 is equal to $\sigma q(x) \dot{W}$, let $\alpha=0.5, k=2, q(x)=\Lambda_{1}\left(0.2 w_{1}+0.2 w_{2}+0.2 w_{3}+0.2 w_{4}\right)$ and $\left(f_{1}, f_{2}\right)$ are component of the in-plane force on boundary along the direction $x, y$, thus the $g_{1}, g_{2}$ in Equation (10h) can be derived by Equations (3) and (4). Since $D$ is rectangle, $g_{2}=0$. Furthermore, let $f_{1}=f_{2}$, the dynamics of simplified Von Karman plate without rotational inertia driven by additive white noise is signified by the motion of the position $x=3, y=2$ of the plate, which are studied in the following cases.

Case I. Let $f_{1}=1.2, \sigma=0.2$; the global random basic attractor, global random point attractor and global random attractors for SAVKP are the same. Figure 2 shows that global random basic attractor is a random fixed point which supports a invariant Markov measure $\delta_{x_{0}}(\omega)$. Furthermore, $\delta_{x_{0}}(\omega)$ is almost surely global stability.

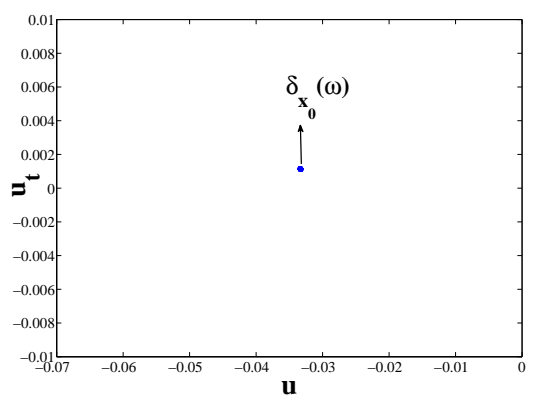

(a)

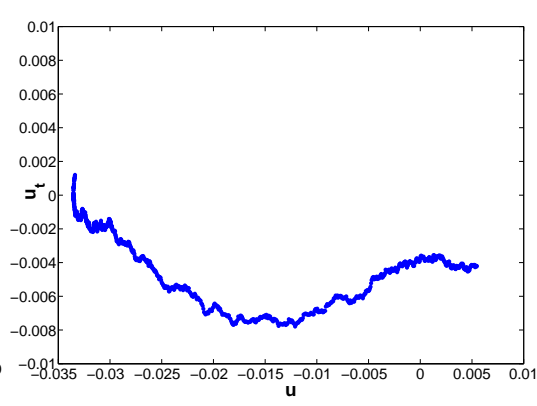

(b)

Figure 2. Invariant measures and random attractors for SAVKP in Case I.: (a) invariant measure and section of global random basic attractor; and (b) global random basic attractor. 
Case II. Set $f_{1}=1.3, \sigma=0.2$, in this situation, global random basic attractor is equivalent to global random point attractor. Global random basic attractor (see Figure 3c) and its section (see Figure 3a) indicate that the system possesses two invariant Markov measures $\delta_{x_{1}}(\omega), \delta_{x_{2}}(\omega)$ which are supported by two fixed random points. Figure $3 b$ illustrates the section of the global random attractor of SAVKP.

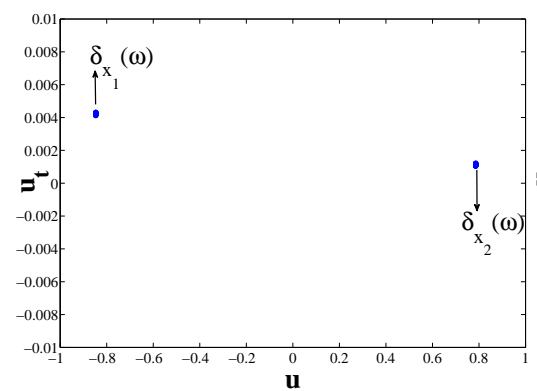

(a)

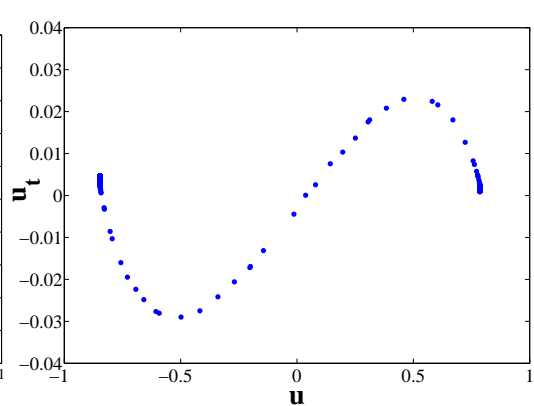

(b)

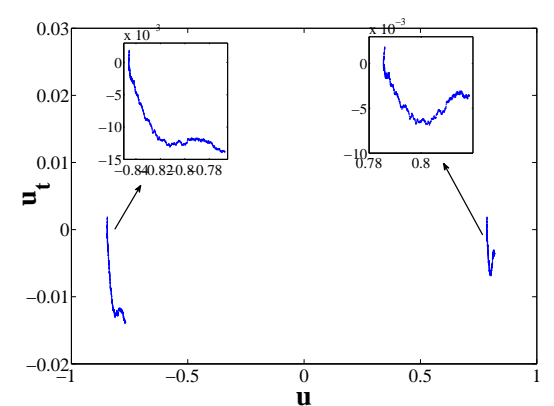

(c)

Figure 3. Invariant measures and random attractors for SAVKP in Case II: (a) invariant measures and global random basic attractor; (b) section of global random attractor; and (c) global random basic attractor.

Case III. let $f_{1}=3, \sigma=0.2$, Figure 4 describes the invariant measures and random attractors for SAVKP. Global random basic attractor is equivalent to global random point attractor in this status. Section of global random basic attractor demonstrated by Figure 4a reveals that the steady states of the system comprise four parts, which means that are least four stable invariant Markov measures for SAVKP exist. In addition, in Figure 4a, it can be obtained that the local dynamics of the system may be complex. The sketch of global random basic attractor is shown by Figure $4 \mathrm{~b}$,

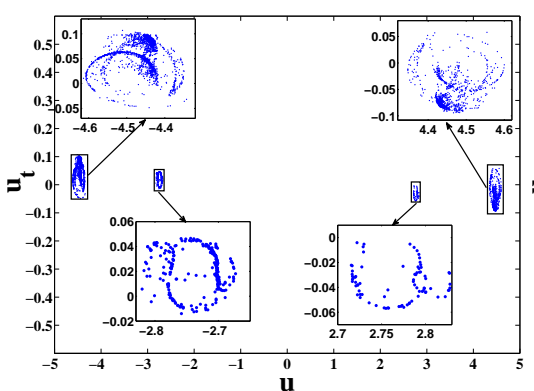

(a)

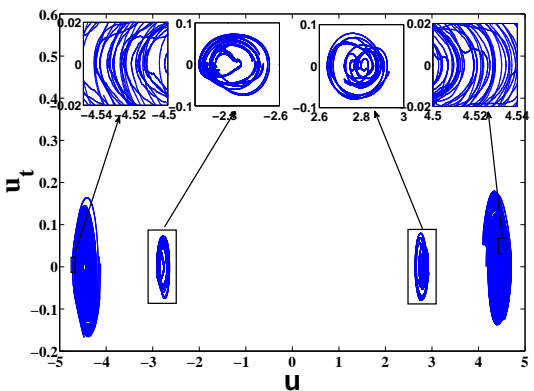

(b)

Figure 4. Invariant measures and random attractors for SAVKP in Case III: (a) invariant measure and section of global random basic attractor; and (b) global random basic attractor. 
Case IV. when $f_{1}=1.3, \sigma=2$, global random basic attractor is also equivalent to global random point attractor in this case. The numerical results on the invariant measures and random attractors for SAVKP (see Figure 5) expose the system has a almost surely global stability invariant Markov measure supported by a random fixed points.

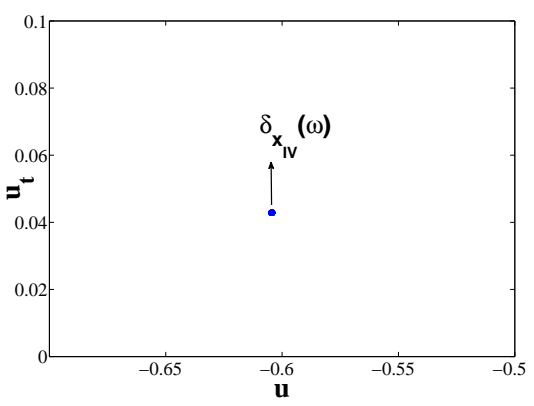

(a)

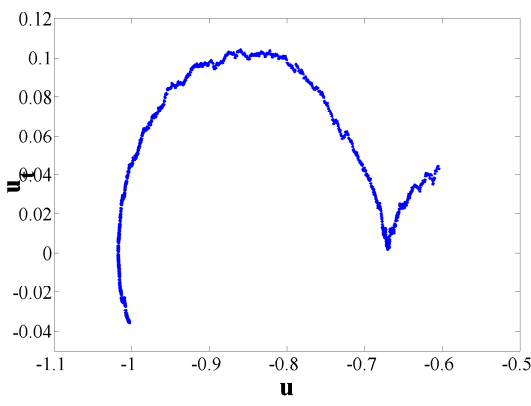

(b)

Figure 5. Invariant measures and random attractors for SAVKP in Case IV: (a) invariant measure and section of global random basic attractor; and (b) global random basic attractor.

The situation of multiplicative white noise. In this case, the $P$ in Figure 1 is equal to $\sigma u \dot{W}$, while the remaining parameters are chosen to be the same as in the situation of additive white noise. The dynamics of SMVKP are studied in the following cases.

Case I. Let $f_{1}=1.1, \sigma=0.02 \Lambda_{1}$, similar to the Case I in additive noise, global random basic attractor, global random point attractor and global random attractors for SAVKP are the same in this circumstance. The assertion that there exists an almost surely global stability invariant measure supported by a random fixed points for SMVKP can be attained by Figure 6 .

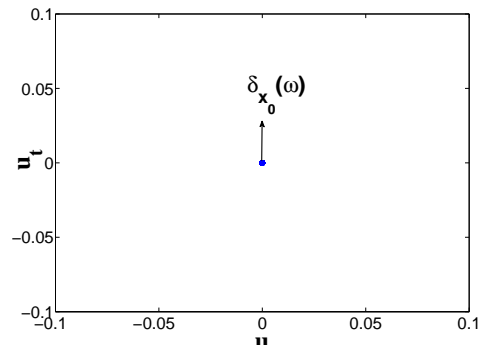

(a)

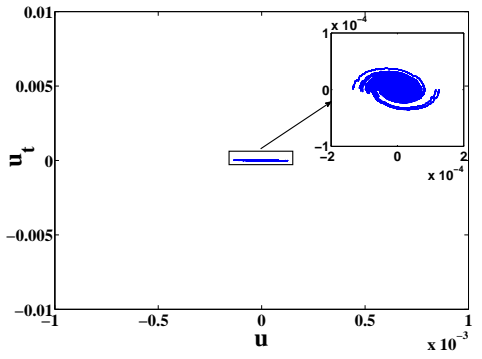

(b)

Figure 6. Invariant measures and random attractors for SMVKP in Case I: (a) invariant measure and section of global random basic attractor; and (b) global random basic attractor.

Case II. When $f_{1}=1.2, \sigma=0.04 \Lambda_{1}$, the numerical results on invariant measures and random attractors were described by Figure 7 . Invoking the section of global random basic attractor (see Figure 7a), it can be obtained that the system possesses two local stable invariant Markov measures, which together with the numerical results of section global random point attractor described by Figure $7 \mathrm{~b}$ give that another invariant measure exists, which could even be a unstable invariant Markov measure.

Case III. Set $f_{1}=2.9, \sigma=0.04 \Lambda_{1}$, Figure 8 expresses the numerical results on global random attractors for SMVKP. The section of global random basic attractor shown by Figure 8a indicates that there exist four local stable invariant Markov measures. Furthermore, SMVKP has another three invariant measures which are interpreted by section of global random point attractor (see Figure $8 b$ ). The sketch of global random point attractor is illustrated by Figure 8c. 
Case IV. Let $f_{1}=1.2, \sigma=0.4 \Lambda_{1}$, the similar results in Case IV can be got, the Figure to describe the invariant measure and random attractor is not displayed here.

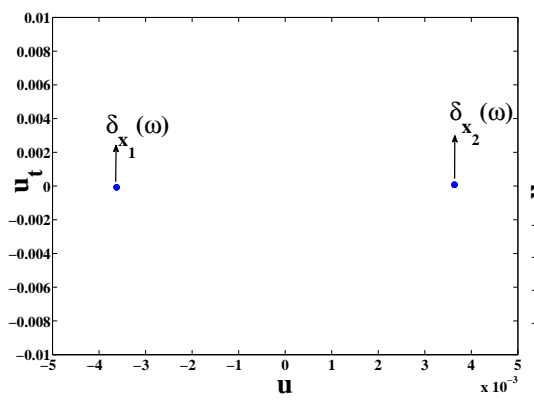

(a)

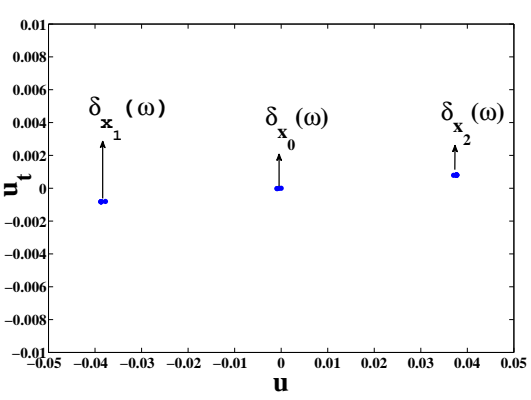

(b)

Figure 7. Invariant measures and random attractors for SMVKP in Case II: (a) invariant measure and section of global random basic attractor; and (b) global random basic attractor.

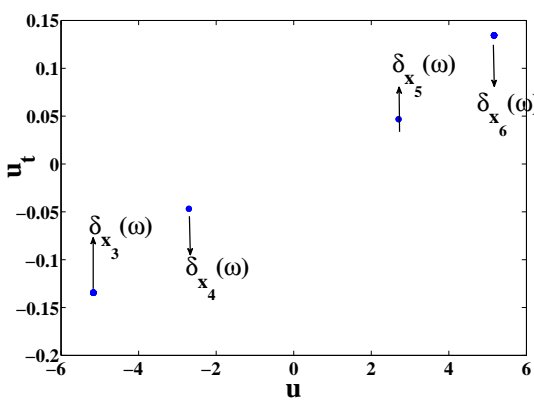

(a)

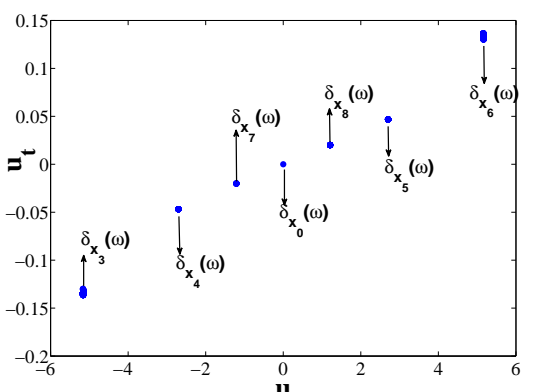

(b)

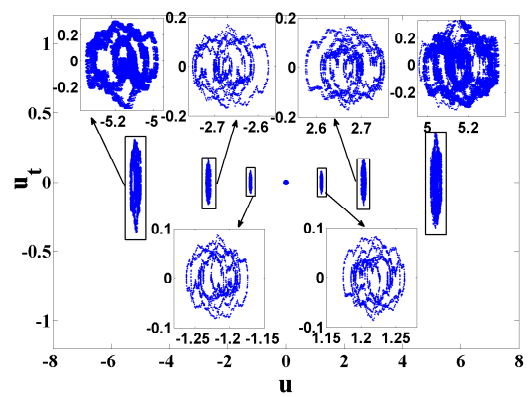

(c)

Figure 8. Invariant measures and random attractors for SMVKP in Case III: (a) invariant measures and global random basic attractor; (b) section of global random point attractor; and (c) global random point attractor.

Some affirmations can be approved by the aforementioned numerical results. For the clamped irrotational inertia Von Karman driven by additive white noise, fixed $\sigma=0.2$, let $f_{1}$ vary from 1.2 to 1.3 , the global $\mathcal{D}$-bifurcation occurs in the motion of the system. Change the value of $f_{1}$ to be a big one, such $f_{1}=3$, the dynamical behavior becomes much more interesting. From the view of global dynamics, there exits secondary $\mathcal{D}$-bifurcation. The local dynamics of the system is complex. On the other hand, let $f_{1}=1.3$ and change the $\sigma$ from 0.2 to 2, the phenomenon of global $\mathcal{D}$-bifurcation disappears. As for clamped irrotational inertia Von Karman driven by multiplicative white noise, fixed $\sigma=0.04 \Lambda_{1}$, the similar global dynamics of the system can be obtained with varying the $f_{1}$ from 1.1 to 2.9. In addition, once the coefficient of the multiplicative white noise becomes big, global $\mathcal{D}$-bifurcation vanishes. Nevertheless, there exist differences between the two cases above. The multiplicative 
white noise is more likely to result in the appearance of global $\mathcal{D}$-bifurcation and secondary global $\mathcal{D}$-bifurcation in the motion of clamped Von Karman without rotational inertia than additive noises. when the secondary $\mathcal{D}$-bifurcation occurs, the local dynamics of the system driven by additive white noise is more complex than the situation of multiplicative white noise.

\section{Preliminary Results}

This section pays attention to give preliminaries and derive certain estimates for solution of SAVKPT1 and SMVKPT1, which are very important to prove main results provided in Section 2.2.

\subsection{Basic Theory Related to Global Random Attractors}

This subsection is devoted to introduce basic theory related to the theory of random attractors used in this paper.

The next Proposition pertinent to $z\left(\theta_{t} \omega\right)$ can be found in References [13,62].

Proposition 1. $z\left(\theta_{t} \omega\right)$ defined by Equation (14) satisfies

$$
\mathbb{E}\left[z\left(\theta_{t} \omega\right)\right]=0, \quad \mathbb{D}\left[z\left(\theta_{t} \omega\right)\right]=\frac{1}{2 \mu},
$$

and $z\left(\theta_{t} \omega\right)$ is a stationary Gauss Process and Markov Process, its probability-distribution function induce a Markov semigroup. Furthermore,

$$
\begin{aligned}
& \lim _{t \rightarrow \pm \infty} \frac{1}{t} \int_{0}^{t}\left|z\left(\theta_{\tau} \omega\right)\right|^{2} d \tau=\mathbb{E}\left[|z(\omega)|^{2}\right]=\frac{1}{2 \mu} \\
& \lim _{t \rightarrow \pm \infty} \frac{1}{t} \int_{0}^{t}\left|z\left(\theta_{\tau} \omega\right)\right| d \tau=\mathbb{E}[|z(\omega)|]=\frac{1}{\sqrt{\pi \mu}}
\end{aligned}
$$

and

$$
\lim _{t \rightarrow \pm \infty} \frac{z\left(\theta_{t} \omega\right)}{t}=0
$$

The sets $\{z(\omega)\}$ is tempered with respect to $\left\{\theta_{t}\right\}_{t \in \mathbb{R}}$. Moreover, if $\mu \geq 2 \beta, \beta>0$, then

$$
\mathbb{E}\left(e^{\beta \int_{s}^{s+t}\left|z\left(\theta_{\tau} \omega\right)\right|^{2} d \tau}\right) \leq e^{\frac{\beta t}{\mu}}, \forall s \in \mathbb{R}, t \geq 0
$$

When $\mu^{3} \geq r^{2}, r \geq 0$, the following holds

$$
\mathbb{E}\left(e^{r \int_{s}^{s+t}\left|z\left(\theta_{\tau} \omega\right)\right| d \tau}\right) \leq e^{\frac{r t}{\sqrt{\mu}}}, \forall s \in \mathbb{R}, t \geq 0
$$

Moreover,

$$
\mathbb{E}\left(\left|z\left(\theta_{s} \omega\right)\right|^{r}\right)=\frac{\Gamma\left(\frac{r+1}{2}\right)}{\sqrt{\pi \mu^{r}}}, r \in \mathbb{R}^{+}
$$

where $\Gamma(\cdot)$ is usual $\Gamma$ function.

To give the notion of global random attractors, the definition of RDS inaugurated by Arnold [4] is needed to be given firstly. 
Definition 1 ([4]). A RDS on Polish space $(X, d)$ with Borel $\sigma$-algebra $\mathcal{B}(X)$ over a metric dynamical system $\left(\Omega, \mathcal{F}, P,\left\{\theta_{t}\right\}_{t \in \mathbb{R}}\right)$ is a $\left(\mathcal{B}\left(\mathbb{R}^{+}\right) \times \mathcal{F} \times \mathbb{B}(X), \mathbb{B}(X)\right)$ measurable mapping

$$
\phi: \mathbb{R}^{+} \times \Omega \times X \rightarrow X, \quad(t, \omega, x) \mapsto \Phi(t, \omega, x)
$$

such that, for $\mathbb{P}-$ a.s., $\omega \in \Omega$,

(i). $\phi(0, \omega)=$ id on $X$.

(ii). $\phi(t+s, \omega)=\phi\left(t, \theta_{s} \omega\right) \circ \phi(s, \omega)$ for all $s, t \in \mathbb{R}^{+}$.

A RDS is continuous or differential if $\phi(t, \omega): X \rightarrow X$ is continuous or differential. Furthermore, $\phi\left(t, \theta_{-t} \omega\right)$ can be understood as the solution start from $-t$ to 0 .

The coming definitions related to random attractors for RDS was established by Crauel et al. [5,63].

Definition 2. A random set $K(\omega)$ is said to absorb the set $B \subset X$ for a $R D S \phi$, if $\mathbb{P}-$ a.s. there exists $t_{B}(\omega)$ such that

$$
\phi\left(t, \theta_{-t} \omega\right) B \subset K(\omega) \text { for all } t \geq t_{B}(\omega)
$$

Definition 3. Let $\mathcal{B} \subset 2^{X}$ be a collection of subsets of $X$, then a closed random set $\mathcal{A}(\omega)$ is called $\mathcal{B}$-random attractor associated with the RDS $\phi$, if $\mathbb{P}-$ a.s.

(i). $\mathcal{A}(\omega)$ is a random compact set.

(ii). $\mathcal{A}(\omega)$ is invariant i.e., $\phi(t, \omega) \mathcal{A}(\omega)=\mathcal{A}\left(\theta_{t} \omega\right)$ for all $t \geq 0$

(iii). For every $B \in \mathcal{B}$,

$$
\lim _{t \rightarrow \infty} \operatorname{dist}\left(\phi\left(t, \theta_{-t} \omega\right) B, \mathcal{A}(\omega)\right)=0
$$

where dist denotes the Hausdorff semidistance:

$$
\operatorname{dis}(A, B)=\sup _{x \in A} \inf _{y \in B} d(x, y), \quad A, B \subset X .
$$

When $\mathcal{B}$ is composed of all bounded set of $X$, then $\mathcal{A}(\omega)$ is the global random attractors for $\phi$. If $\mathcal{B}=\{\{x\}: x \in X\}, \mathcal{A}(\omega)$ is said to be global random point attractor.

The next theorem, dedicated to verifying the existence of random attractors for SEBT1 directly, can be derived from Theorem 3.11 in Crauel et al. [5].

Theorem 5. Suppose $\phi$ is an RDS on connected Polish space $(X, d)$, and suppose that $\phi$ possesses an absorbing set in $X$ and for any nonrandom bounded set $B \subset X, \lim _{t \rightarrow+\infty} \phi\left(t, \theta_{-t} \omega\right) B$ is relatively compact $\mathbb{P}$-a.s. Then, $\phi$ possesses uniqueness global random attractors defined by the following

$$
A(\omega)=\overline{\bigcup_{B \subset X} \Lambda_{B}(\omega)}
$$

where union is taken over all bounded $B \subset X$, and $\Lambda_{B}(\omega)$ given by

$$
\Lambda_{B}(\omega)=\bigcap_{s \geq 0} \overline{\bigcup_{t \geq s} \phi\left(t, \theta_{-t} \omega\right) B}
$$

Furthermore, $\mathscr{A}(\omega)$ is measurable with respect to $\mathcal{F}$ and connected.P - a.s..

The following assertion provides the relationship between random attractors and invariant measures which is important to expound the global dynamics for RDS was stated in $[4,8,63,64]$. 
Proposition 2. When the RDS $\varphi$ possesses global random attractor comply with Definition 3, by the Corollary 4.4 in Crauel [8], this attractors supports every invariant measures. The random point attractor of $\varphi$ given by Definition 3 always supports at lest one invariant measure which even is a invariant Markov measure (Crauel [63], P423; Arnold [4], Theorem 1.6.13 and Theorem 1.7.5). When $\varphi$ is a white noise RDS or SDS, together with the Theorem 3.6 in Crauel [63] give that every invariant Markov measure is supported by the global point attractor. On the other hand, if the global random attractors for $\varphi$ exists, then $\varphi$ also has the global point attractor. For any fixed $\omega \in \Omega$, taking advantage of pullback mechanism [4], follow the proof of Theorem 5.2 in Birnir [64], the global random attractor can be decomposed into two ingredients, one is random basic attractor which supports all stable invariant Markov measures of white noise RDS, the other is random remainder. For the definition of basic and remainder, we refer to Birnir [64].

With the assertion that the RDS possesses a global random attractors, in light of Proposition 2, the investigation on global dynamics of RDS can be accomplished by exploiting the numerical results on the structure of global random attractor.

The next proposition should be used in checking that the $\operatorname{RDS} \phi(t, \omega)$ satisfies condition "for any nonrandom bounded set $B \subset X, \lim _{t \rightarrow+\infty} \phi\left(t, \theta_{-t} \omega\right) B$ is relative compact $\mathbb{P}$-a.s." in Theorem 5 .

Proposition 3. ([26]) Suppose $B \subset X$ is any bounded set, let

$$
\alpha(B)=\inf \{d: B \text { has a finite cover of diameter }<d\}
$$

then $\alpha(B)$ is known as the Kuratowski's $\alpha$-measure of non-compactness of $B$, in short, $\alpha$-measure of $B$, which has the following properties.

(i) $\alpha(B)=0$ if and only if $B$ is pre-compact.

(ii) $\alpha(A \cup B) \leq \max \{\alpha(A), \alpha(B)\}$.

(iii) $\alpha(A+B) \leq \alpha(A)+\alpha(B)$.

(iv) $\alpha(\operatorname{coB} B)=\alpha(B)$, where $\alpha(\operatorname{coB} B)$ is the closed convex hull of $B$.

(v) If $B_{1} \supset B_{2} \supset B_{3} \supset \cdots$ are nonempty closed sets in $X$ such that $\alpha\left(B_{n}\right) \rightarrow 0$ as $n \rightarrow \infty$, then $\cap_{n \geq 1} B_{n}$ is nonempty and compact.

\subsection{Main Estimates}

This subsection presents the main estimates for the solutions of the systems and some lemmas that are momentous to derive the proof for main results.

Firstly, the following properties on Von Karman bracket were given by Proposition 1.4.2 in Chueshov and Lasiecka [26].

If either at least on of $u, v, w$ belongs to $H_{0}^{2}(D)$ or all of them belong to $\left(H_{0}^{1} \cap H^{2}\right)(D)$, then

$$
\int_{D}[u, v] w d x=\int_{D}[u, w] v d x=\int_{D}[v, w] u d x
$$

If $v, w \in\left(H_{0}^{1} \cap H^{2}\right)(D)$, then

$$
\int_{D}[u, v] w d x=\int_{D}[u, w] v d x, u \in H^{2}(D) .
$$

The next Lemma consider the sharp regularity Von Karman bracket.

Lemma 1. ([25]) Suppose $D$ is a bounded domain with regular boundary or a rectangle in $\mathbb{R}^{2}$, the following estimates holds

$$
\|[u, w]\|_{-j-\theta} \leq C\|u\|_{2-\theta+\beta}\|w\|_{3-j-\beta}, \quad u \in H^{2-\theta+\beta}(D), w \in H^{3-j-\beta}(D),
$$


where $j=0,1,0<\beta \leq \theta<1$.

$$
\|[u, w]\|_{-j} \leq C\|u\|_{2-\beta}\|w\|_{3-j+\beta}, u \in H^{2-\beta}(D), w \in H^{3-j+\beta}(D),
$$

in which $j=1,2,0 \leq \beta<1$. Let $\Delta^{-2}$ be the inverse operator of $\Delta^{2}$ with clamped boundary condition, invoking Equations (41) and (42), we have $(u, w) \mapsto G(u, w) \equiv \Delta^{-2}[u, w]$ is bounded mapping from $\left(H^{2} \times H^{2}\right)(D)$ to $\left(H^{3} \cap W^{2, \infty}\right)(D)$ which satisfies

$$
\begin{aligned}
& \|[u, w]\|_{-2} \leq C\|u\|_{1}\|w\|_{2} \\
& \|G(u, v)\|_{2, \infty} \leq C\|u\|_{2}\|v\|_{2} \\
& \|[w, G(u, v)]\|_{2} \leq C\|u\|_{2}\|v\|_{2}\|w\|_{2} .
\end{aligned}
$$

The assertion that the condition " $D$ is a bounded domain with regular boundary or a rectangle in

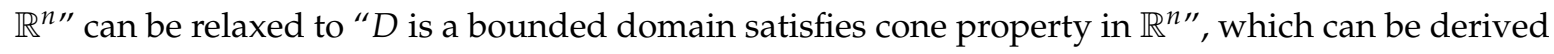
using the property of continuation in Sobolev space [65].

The coming Lemma reveals a relationship between Airy function $\mathcal{F}$ and in-plane force $F_{0}$.

Lemma 2. Let $\mathcal{F}$ is the solution for system described by Equations (10e) and (10f); $F_{0}$ satisfies Equations (10g) and (10h); and then

$$
\int_{D} \Delta \mathcal{F} \Delta F_{0} d x=0
$$

Proof. See the Appendix B.1.

According to Lemma 2, the following estimates which play a crucial role in obtaining the existence of global absorbing set for vibration of Von Karman with a arbitrarily small coefficient of weakly damping.

Let $\boldsymbol{\Phi}=[u, v]^{T}$ be the solution of SAVKPT2 or SMVKPT2, $\varepsilon$ satisfies (23), define

$$
\mathrm{Y}\left(\mathcal{F}, F_{0}\right)=\int_{D}[u, u] F_{0} d x
$$

Combining Lemma 2 with Equations (10e)-(10h), we have

$$
\mathrm{Y}\left(\mathcal{F}, F_{0}\right)=\int_{D} \Delta^{2} \mathcal{F}(u) F_{0} d x=\int_{\Gamma} \frac{\partial \Delta \mathcal{F}}{\partial n} F_{0} d s-\int_{\Gamma} \frac{\partial F_{0}}{\partial n} \Delta \mathcal{F} d s,
$$

then

$$
\begin{aligned}
\mathrm{Y}\left(\mathcal{F}, F_{0}\right) & \leq\left\|\frac{\partial \Delta \mathcal{F}}{\partial n}\right\|_{-\frac{3}{2}, \Gamma}\left\|F_{0}\right\|_{\frac{3}{2}, 0, \Gamma}+\left\|\frac{\partial F_{0}}{\partial n}\right\|_{\frac{1}{2}, \Gamma}\|\Delta \mathcal{F}\|_{-\frac{1}{2}, 0^{\prime}} \\
& \leq C_{1}\left\|\frac{\partial \Delta \mathcal{F}}{\partial n}\right\|_{-\frac{3}{2}, \Gamma}\left\|F_{0}\right\|_{\frac{3}{2}, 0, \Gamma}+C_{2}\left\|\frac{\partial F_{0}}{\partial \eta}\right\|_{\frac{1}{2}, \Gamma}\|\Delta \mathcal{F}\|_{-\frac{1}{2}, 0, \Gamma^{\prime}} \\
& \leq \eta\|\Delta \mathcal{F}\|^{2}+C\left(\eta, C_{1}, C_{2}\right)\left\|F_{0}\right\|_{2}^{2} .
\end{aligned}
$$

where $\eta$ is any given positive, $C\left(\eta, C_{1}, C_{2}\right)$ is a positive, which is inversely proportional to $\eta$. In addition, invoking the trace theorem [57], we attain

$$
\left\|F_{0}\right\|_{2}^{2} \leq \widetilde{C}\left\|g_{1}\right\|_{\frac{3}{2}, \Gamma}^{2}+\widehat{C}\left\|g_{2}\right\|_{\frac{1}{2}, \Gamma}^{2} .
$$

In contrast to Inequality (9.1.17) provided by Lemma 9.1.7 in Chueshov and Lasiecka [26], the inequality in Equation (45) give a clear relationship between $Y\left(\mathcal{F}, F_{0}\right)$ and the in-plane force $F_{0}$, which along with Equation (46) indicates that $Y\left(\mathcal{F}, F_{0}\right)$ is determined by the component of the in-plane force on boundary along the direction $x, y$.

Since the form of damping is weak and the coefficient of it can be arbitrarily small, it is 
accomplished by verifying the condition in Theorem 5 that "for any nonrandom bounded set $B \subset E_{1}, \lim _{t \rightarrow+\infty} \phi\left(t, \theta_{-t} \omega\right) B$ is relative compact $\mathbb{P}$-a.s." relies on the stabilization estimation of the considered systems. The results listed in the next two Lemmas are very important to accomplish the stabilization estimation of SAVKPT1 and SMVKPT1.

Lemma 3. Suppose $\left\{u^{n}\right\}_{n=1}^{+\infty} \in L^{\infty}\left((\tau, t),\left(H_{0}^{2} \cap H^{4}\right)(D),\left\{u_{t}^{n}\right\}_{n=1}^{+\infty} \in L^{\infty}\left((\tau, t), L^{2}(D)\right.\right.$ are two given bounded sequences, which are weakly star convergence to $u, u_{t}$, respectively. Then, for $\forall n, m \in \mathbb{N}$, the following holds

$$
\lim _{n \rightarrow \infty} \lim _{m \rightarrow \infty} \int_{\tau}^{t}\left(\left[\mathcal{F}\left(u^{n}\right)+F_{0}, u^{n}\right]-\left[\mathcal{F}\left(u^{m}\right)+F_{0}, u^{m}\right], u_{t}^{n}-u_{t}^{m}\right) d s=0 .
$$

Lemma 4. Suppose $\left\{u^{n}\right\}_{n=1}^{+\infty} \in L^{\infty}\left((\tau, t),\left(H_{0}^{2} \cap H^{4}\right)(D),\left\{u_{t}^{n}\right\}_{n=1}^{+\infty} \in L^{\infty}\left((\tau, t), L^{2}(D)\right.\right.$ are two given bounded sequences, which are weakly star convergence to $u, u_{t}$, respectively. Then, for $\forall n, m \in \mathbb{N}$, the following holds

$$
\lim _{n \rightarrow \infty} \lim _{m \rightarrow \infty} \int_{\tau}^{t}\left(\left[\mathcal{F}\left(u^{n}\right)+F_{0}, u^{n}\right]-\left[\mathcal{F}\left(u^{m}\right)+F_{0}, u^{m}\right], u^{n}-u^{m}\right) d s=0 .
$$

Lemma 3 was proposed by Khanmamedov [35]. With a similar treatment, the results in Lemma 4 can be attained. Hence, it is omitted it here.

It is noticed that the $\mathbf{A}_{1}$ in Equations (15) and (20) are the same, then the estimates given in the next Lemma, which can be obtained by simple computation, should be employed in both cases of additive white noise and multiplicative white noise.

Lemma 5. for any $\mathbf{U}=\left[U_{1}, U_{2}\right]^{T} \in E_{1}$, the ensuring holds

$$
\left(\mathbf{A}_{1} \mathbf{U}, \mathbf{U}\right)_{E_{1}} \leq-\frac{\varepsilon}{2}\|\mathbf{U}\|_{E_{1}}^{2}-\frac{k_{0}}{2}\left\|U_{2}\right\|
$$

where $0<\varepsilon<\varepsilon_{0}, 0<k_{0} \leq \alpha-\varepsilon$ and $\varepsilon, \varepsilon_{0}$ satisfies Equation (23).

The following Lemmas are used to prove the existence and uniqueness of solutions for SAVKP and SMVKP by semigroup theory.

\section{Lemma 6.}

$$
-\mathbf{A}_{1} \in \mathscr{L}\left(E_{2}, E_{1}\right), \quad\left(-\mathbf{A}_{1}\right)^{-1} \in \mathscr{L}\left(E_{1}, E_{1}\right) .
$$

Proof. See Appendix B.2.

Base on the Lemma 6, the ensuring results focus on eigenvalues of $\mathbf{A}_{1}$ can be verified.

Lemma 7. The eigenvalues of $\mathbf{A}_{1}$ are as follows.

$$
\begin{aligned}
& \lambda_{k}^{(+)}=\frac{-\alpha+\sqrt{\alpha^{2}-4 \Lambda_{k}}}{2}, \\
& \lambda_{k}^{(-)}=\frac{-\alpha-\sqrt{\alpha^{2}-4 \Lambda_{k}}}{2}, k=1, \cdots, n, \cdots .
\end{aligned}
$$

Proof. See Appendix B.3. 
3.2.1. Main Estimates Only Be Valid in Situation of Additive White Noise

This subsubsection is to give main estimates that only be valid in additive white noise case.

For any given $T>0, \tau \in \mathbb{R}$, for $\forall \mathbf{U}, \mathbf{V} \in E_{1},\|\mathbf{U}\|_{E_{1}} \leq M,\|\mathbf{V}\|_{E_{1}} \leq M$, by Reference [26], we have there exists constant $M=C(M)$, such that

$$
\left[\mathcal{F}\left(U_{1}\right)+F_{0}, U_{1}\right]-\left[\mathcal{F}\left(V_{1}\right)+F_{0}, V_{1}\right] \leq C(M)\|\mathbf{U}-\mathbf{V}\|_{E_{1}}
$$

along with Equation (50), we have

$$
\left\|\mathbf{F}_{3}(\mathbf{U})-\mathbf{F}_{3}(\mathbf{V})\right\|_{E_{1}} \leq C_{1}(T, \tau, \omega, M)\|\mathbf{U}-\mathbf{V}\|_{E_{1}}
$$

where $C_{1}(T, \tau, \omega, M)$ is a constant.

Let the value of $\eta$ in Equation (45) be $\frac{7}{6}, \frac{1}{2}, \frac{1}{4}$, respectively, we find

$$
\begin{aligned}
\frac{3 k \varepsilon}{2} Y\left(\mathcal{F}, F_{0}\right) Y\left(\mathcal{F}, F_{0}\right) \leq & \frac{7 k \varepsilon}{4}\|\Delta \mathcal{F}\|^{2}+\frac{3 k \varepsilon}{2} C\left(\frac{7}{6}, C_{1}, C_{2}\right)\left\|F_{0}\right\|_{2}^{2} \\
k Y\left(\mathcal{F}, F_{0}\right) Y\left(\mathcal{F}, F_{0}\right) \leq & \frac{k}{2}\|\Delta \mathcal{F}\|^{2}+\frac{k}{2} C\left(\frac{1}{2}, C_{1}, C_{2}\right)\left\|F_{0}\right\|_{2}^{2} \\
\sqrt{\bar{k}}\left|\sigma z\left(\theta_{t} \omega\right)\right|\|q\| k Y\left(\mathcal{F}, F_{0}\right) \leq & \sqrt{\bar{k}}\left|\sigma z\left(\theta_{t} \omega\right)\right|\|q\| \frac{k}{4}\|\Delta \mathcal{F}\|^{2} \\
& +\sqrt{\bar{k}} k\left|\sigma z\left(\theta_{t} \omega\right)\right|\|q\| C\left(\frac{1}{4}, C_{1}, C_{2}\right)\left\|F_{0}\right\|_{2}^{2} .
\end{aligned}
$$

Applying Equations (39) and (40) and Lemma 1, we get

$$
\begin{aligned}
& \int_{D}[\mathcal{F}(u), u] v d x \\
= & \int_{D}\left[u_{t}, u\right] \mathcal{F}(u) d x+\varepsilon \int_{D}[u, u] \mathcal{F}(u) d x-\sigma z\left(\theta_{t} \omega\right) \int_{D}[q, u] \mathcal{F}(u) d x \\
\leq & -\frac{1}{2} \int_{D}\left(\Delta^{2} \mathcal{F}(u)\right)_{t} \mathcal{F}(u) d x-\varepsilon \int_{D} \Delta^{2} \mathcal{F}(u) \mathcal{F}(u) d x+\left|\sigma z\left(\theta_{t} \omega\right)\right| \int_{D}[q, u] \mathcal{F}(u) d x \\
\leq & -\frac{1}{4} \frac{d}{d t}\|\Delta \mathcal{F}(u)\|^{2}-\varepsilon\|\Delta \mathcal{F}(u)\|^{2}+\frac{\left|\sigma z\left(\theta_{t} \omega\right)\right|\|q\|_{1}}{\sqrt{k}}\left(\left\|A^{\frac{1}{2}} u\right\|^{2}+\frac{k}{4}\|\Delta \mathcal{F}(u)\|^{2}\right)
\end{aligned}
$$

and

$$
\begin{aligned}
& \int_{D}\left[F_{0}, u\right] v d x \\
= & \int_{D}\left[u_{t}, u\right] F_{0} d x+\varepsilon \int_{D}[u, u] F_{0} d x-\sigma z\left(\theta_{t} \omega\right) \int_{D}[q, u] F_{0} d x \\
\leq & -\frac{1}{2} \int_{D}\left(\Delta^{2} \mathcal{F}(u)\right)_{t} F_{0} d x-\varepsilon \int_{D} \Delta^{2} \mathcal{F}(u) F_{0} d x+\left|\sigma z\left(\theta_{t} \omega\right)\right| \int_{D}[q, u] F_{0} d x \\
= & -\frac{1}{2} \frac{d}{d t} Y\left(\mathcal{F}, F_{0}\right)-\varepsilon Y\left(\mathcal{F}, F_{0}\right)+\frac{\eta}{2}\|A u\|^{2}+\frac{1}{2 \eta}\left(\left|\sigma z\left(\theta_{t} \omega\right)\right|\|q\|_{1}\left\|F_{0}\right\|_{2}\right)^{2} .
\end{aligned}
$$

where $\eta$ is positive constant. Set $\eta=\frac{\varepsilon}{4 k}$, then

$$
\begin{aligned}
& \int_{D}\left[F_{0}, u\right] v d x \\
\leq & -\frac{1}{2} \mathrm{Y}\left(\mathcal{F}, F_{0}\right)-\varepsilon Y\left(\mathcal{F}, F_{0}\right)+\frac{\varepsilon}{8 k}\|A u\|^{2}+\frac{2 k}{\varepsilon}\left(\mid \sigma z\left(\theta_{t} \omega\right)\|q q\|_{1}\left\|F_{0}\right\|_{2}\right)^{2} .
\end{aligned}
$$


Employing Young's inequality, it can be obtained that

$$
\begin{array}{r}
2\left(\sigma A^{\frac{1}{2}} q(x) z\left(\theta_{t} \omega\right), A^{\frac{1}{2}} u\right) \leq \frac{\varepsilon}{4}\left\|A^{\frac{1}{2}} u\right\|^{2}+\frac{4 \sigma^{2}\left|z\left(\theta_{t} \omega\right)\right|^{2}}{\varepsilon}\left\|A^{\frac{1}{2}} q(x)\right\|^{2}, \\
2\left(\sigma(\mu-\alpha+\varepsilon) q(x) z\left(\theta_{t} \omega\right), v\right) \leq \frac{k_{0}}{2}\|v\|^{2}+\frac{\left(\sigma(\mu-\alpha+\varepsilon) z\left(\theta_{t} \omega\right)\right)^{2}}{k_{0}}\|q(x)\|^{2} .
\end{array}
$$

The next lemma shows $S^{A}(t, \omega)$ possesses global random absorbing set in $E_{1}$. In addition, the expectation of radius of this random set is bounded.

Lemma 8. For any given non random bounded set $B \subset E_{1}$, there exists $T_{B}(\omega) \geq 0$, such that, for $\forall t \geq T_{B}(\omega)$, the following holds $\mathbb{P}$-a.s.

$$
\left\|S^{A}\left(t, \theta_{-t} \omega\right) B\right\|_{E_{1}} \leq r(\omega)
$$

and $\mathbb{E}(\rho(\omega)) \leq M<\infty$. In which $\rho(\omega)$ and $r(\omega)$ are formulated by Equations (26) and (27), respectively.

Proof. See Appendix B.4.

Let $K(\omega)=\mathscr{B}(0, r(\omega))$; based on Lemma 8, it can be obtained that, for any non-random bounded set $B \subset E_{1}$, there exists $T_{B}(\omega)>0$, such that, for $\forall T_{1} \geq T_{B}(\omega)$, the following holds

$$
S^{A}\left(T_{1}, \theta_{-T_{1}} \omega\right) B \subset K(\omega) .
$$

The coming lemma plays a key role in verifying the condition in Theorem 5 that "for any nonrandom bounded set $B \subset E_{1}, \lim _{t \rightarrow+\infty} \phi\left(t, \theta_{-t} \omega\right) B$ is relative compact $\mathbb{P}$-a.s." which is significant to prove the existence of the global random attractors for SAVKPT1.

Lemma 9. Suppose $\left\{\mathbf{U}_{n}^{(0)}\right\}_{n=1}^{+\infty}$ is any given bounded sequence in any given non-random bounded set $B \subset E_{1}$, $T_{0} \in[0, \infty)$ is a any given constant, $n, m \in \mathbb{N}$. Then, for $\forall \bar{\varepsilon}>0$, there exist $T_{B}(\omega, \bar{\varepsilon}) \geq T_{B}(\omega)$ and $T=\max \left\{T_{B}(\omega, \bar{\varepsilon}), T_{0}\right\}$ such that

$$
\left\|S^{A}\left(T, \theta_{-T} \omega\right) \mathbf{U}_{m}^{(0)}-S^{A}\left(T, \theta_{-T} \omega\right) \mathbf{U}_{n}^{(0)}\right\|_{E_{1}} \leq \bar{\varepsilon}+K_{\bar{\varepsilon}, B, T, \omega}^{A}\left(\mathbf{U}_{m}^{(0)}, \mathbf{U}_{n}^{(0)}\right),
$$

and

$$
\liminf _{n \rightarrow+\infty} \liminf _{m \rightarrow+\infty} K_{\bar{\varepsilon}, B, T, \omega}^{A}\left(\mathbf{U}_{m}^{(0)}, \mathbf{U}_{n}^{(0)}\right)=0 .
$$

Proof. See the Appendix B.5.

\subsubsection{Main Estimates Only Be Valid in Situation of Multiplicative White Noise}

For any given $T>0, \tau \in \mathbb{R}, \forall \mathbf{U}, \mathbf{V} \in E_{1},\|\mathbf{U}\|_{E_{1}} \leq M,\|\mathbf{V}\|_{E_{1}} \leq M, M$ is constant, take account into $z\left(\theta_{t} \omega\right)$ is continuous in $[\tau, \tau+T]$, merging with Equation (50), we have that there exists a constant $C(T, \tau, \omega, M)$ such that

$$
\left\|\mathbf{F}_{2}\left(\mathbf{U}, \theta_{t} \omega\right)+\mathbf{F}_{3}(\mathbf{U})-\mathbf{F}_{2}\left(\mathbf{V}, \theta_{t} \omega,\right)-\mathbf{F}_{3}(\mathbf{V})\right\|_{E_{1}} \leq C(T, \tau, \omega, M)\|\mathbf{U}-\mathbf{V}\|_{E_{1}},
$$


which indicates that $\mathbf{F}_{2}\left(\mathbf{U}, \theta_{t} \omega\right)+\mathbf{F}_{3}(\mathbf{U}): E_{1} \rightarrow E_{1}$ satisfies Lipschtiz condition.

Let $\boldsymbol{\Phi}=\left[\Phi_{1}, \Phi_{2}\right]^{T}=[u, v]^{T} \in E_{2}$; the following estimates should be frequently used.

$$
\begin{gathered}
\left(\sigma z\left(\theta_{t} \omega\right) u, u\right)_{2} \leq\left|\sigma z\left(\theta_{t} \omega\right)\right|\|u\|_{2}^{2}, \\
\left(-\sigma z\left(\theta_{t} \omega\right) v, v\right) \leq\left|\sigma z\left(\theta_{t} \omega\right)\right|\|v\|_{2}^{2} \\
\left(\sigma^{2} z^{2}\left(\theta_{t} \omega\right) u, v\right) \leq \frac{\sigma^{2}\left|z\left(\theta_{t} \omega\right)\right|^{2}}{2 \sqrt{\lambda_{1}}}\left(\|A u\|^{2}+\|v\|^{2}\right) . \\
\int_{D}[\mathcal{F}(u), u] v d x \\
=-\frac{1}{2} \int_{D}\left(\Delta^{2} \mathcal{F}(u)\right)_{t} \mathcal{F}(u) d x-\varepsilon \int_{D} \Delta^{2} \mathcal{F}(u) \mathcal{F}(u) d x+\sigma z\left(\theta_{t} \omega\right) \int_{D}[u, u] \mathcal{F}(u) d x \\
=-\frac{1}{4} \frac{d}{d t}\|\Delta \mathcal{F}(u)\|^{2}-\varepsilon\|\Delta \mathcal{F}(u)\|^{2}+\sigma z\left(\theta_{t} \omega\right)\|\Delta \mathcal{F}(u)\|^{2} \\
\int_{D}\left[F_{0}, u\right] v d x \\
=\int_{D}\left[u_{t}, u\right] F_{0} d x+\varepsilon \int_{D}[u, u] F_{0} d x-\sigma z\left(\theta_{t} \omega\right) \int_{D}[u, u] F_{0} d x \\
=-\frac{1}{2} \frac{d}{d t} \mathrm{Y}\left(\mathcal{F}, F_{0}\right)-\varepsilon Y\left(\mathcal{F}, F_{0}\right)+\sigma z\left(\theta_{t} \omega\right) Y\left(\mathcal{F}, F_{0}\right) d x
\end{gathered}
$$

where $Y\left(\mathcal{F}, F_{0}\right)$ is defined by Equation (44).

Let the value of $\eta$ in Equation (45) be $\frac{1}{8}$ and $\frac{3}{2}$, respectively, and the following can be satisfied

$$
\begin{aligned}
& 4\left(2\left|\sigma z\left(\theta_{t} \omega\right)\right|+\frac{\sigma^{2}\left|z\left(\theta_{t} \omega\right)\right|^{2}}{\sqrt{\Lambda_{1}}}\right) k Y\left(\mathcal{F}, F_{0}\right) \\
\leq & \frac{1}{2}\left(2\left|\sigma z\left(\theta_{t} \omega\right)\right|+\frac{\sigma^{2}\left|z\left(\theta_{t} \omega\right)\right|^{2}}{\sqrt{\Lambda_{1}}}\right) k\|\Delta \mathcal{F}\|^{2} \\
& +4\left(2\left|\sigma z\left(\theta_{t} \omega\right)\right|+\frac{\sigma^{2}\left|z\left(\theta_{t} \omega\right)\right|^{2}}{\sqrt{\Lambda_{1}}}\right) k C\left(\frac{1}{8}, C_{1}, C_{2}\right)\left\|F_{0}\right\|_{2}^{2}, \\
& \varepsilon k Y\left(\mathcal{F}, F_{0}\right) \leq \frac{3}{2} \varepsilon k\|\Delta \mathcal{F}\|^{2}+\varepsilon k C\left(\frac{3}{2}, C_{1}, C_{2}\right)\left\|F_{0}\right\|_{2}^{2},
\end{aligned}
$$

The coming Lemma asserts that $S^{M}(t, \omega)$ possesses global random absorbing set in $E_{1}$.

Lemma 10. Let $B \subset E_{1}$ be any given non-random bounded set, then there exists $T_{B}(\omega) \geq 0$ such that

$$
\left\|S^{M}\left(t, \theta_{-t} \omega\right) B\right\|_{E_{1}} \leq r(\omega), \quad \forall t \geq T_{B}(\omega), \mathbb{P}-\text { a.s. }
$$

and

$$
\mathbb{E}(\rho(\omega)) \leq M<\infty
$$

where $\rho(\omega), r(\omega)$ is formulated by Equations (30) and (32), respectively. $M$ is given in Theorem 4.

Proof. See Appendix B.6.

Let $K(\omega)=\mathscr{B}(0, r(\omega))$, invoke Lemma 10 , we have that for any given bounded set $B \subset E_{1}$, there exists $T_{B}(\omega)>0$, such that for $\forall T_{1} \geq T_{B}(\omega)$, the coming holds

$$
S^{M}\left(T_{1}, \theta_{-T_{1}} \omega\right) B \subset K(\omega) .
$$

Similar to the results presented in Lemma 9, the stabilization estimation of SMVKPT1 provided in the next Lemma can be derived. 
Lemma 11. Suppose $\left\{\mathbf{U}_{n}^{(0)}\right\}_{n=1}^{+\infty}$ is any given bounded sequence in any given non-random bounded set $B \subset E_{1}$, $T_{0} \in[0, \infty)$ is a any given constant, $n, m \in \mathbb{N}$. Then, for $\forall \bar{\varepsilon}>0$, there exist $T_{B}(\omega, \bar{\varepsilon}) \geq T_{B}(\omega)$ and $T=\max \left\{T_{B}(\omega, \bar{\varepsilon}), T_{0}\right\}$ such that

$$
\left\|S^{M}\left(T, \theta_{-T} \omega\right) \mathbf{U}_{m}^{(0)}-S^{M}\left(T, \theta_{-T} \omega\right) \mathbf{U}_{n}^{(0)}\right\|_{E_{1}} \leq \bar{\varepsilon}+K_{\bar{\varepsilon}, B, T, \omega}^{M}\left(\mathbf{U}_{m}^{(0)}, \mathbf{U}_{n}^{(0)}\right),
$$

and

$$
\liminf _{n \rightarrow+\infty} \liminf _{m \rightarrow+\infty} K_{\bar{\varepsilon}, B, T, \omega}^{M}\left(\mathbf{U}_{m}^{(0)}, \mathbf{U}_{n}^{(0)}\right)=0 .
$$

Proof. See Appendix B.7.

\section{Proofs for Main Results}

\subsection{Proof for Theorem 1}

Along with Lemmas 5-7, we have $\mathbf{A}_{1}$ can induce a linear semigroup of contractions denoted by $e^{\mathbf{A}_{1}{ }^{t}, t}, \in \mathbb{R}^{+}$. Invoking Equation (51), we get $\mathbf{F}_{2}\left(\theta_{t} \omega\right)+\mathbf{F}_{3}(\mathbf{U}): E_{1} \rightarrow E_{1}$ satisfies the Lipschtiz condition. $z\left(\theta_{t} \omega\right)$ is continuous in $[\tau, t]$. Hence, according to Theorem 2.5.1 in Reference [66], we conclude that SAVKP possesses uniqueness (mild) solution with the form

$$
\mathbf{\Phi}^{A}\left(t, \tau, \omega ; \boldsymbol{\Phi}_{\tau}\right)=e^{\mathbf{A}(t-\tau)} \mathbf{\Phi}_{\tau}+\int_{\tau}^{t} e^{\mathbf{A}(t-s)}\left(\mathbf{F}_{2}\left(\theta_{s} \omega\right)+\mathbf{F}_{3}(\mathbf{\Phi}(s))\right) d s .
$$

where, $t \geq \tau, t, \tau \in \mathbb{R}$. Thus,

$$
\begin{aligned}
& \boldsymbol{\Phi}^{A}\left(t-\tau, 0, \theta_{\tau} \omega ; \mathbf{\Phi}_{\tau}\right) \\
= & e^{\mathbf{A}(t-\tau)} \boldsymbol{\Phi}_{\tau}+\int_{0}^{t-\tau} e^{\mathbf{A}(t-\tau-s)}\left(\mathbf{F}_{2}\left(\theta_{s+\tau} \omega\right)+\mathbf{F}_{3}(\boldsymbol{\Phi}(s))\right) d s .
\end{aligned}
$$

Let $s+\tau=T$, we have

$$
\begin{aligned}
& e^{\mathbf{A}(t-\tau)} \boldsymbol{\Phi}_{\tau}+\int_{0}^{t-\tau} e^{\mathbf{A}(t-\tau-s)}\left(\mathbf{F}_{2}\left(\theta_{s+\tau} \omega\right)+\mathbf{F}_{3}(\boldsymbol{\Phi}(s))\right) d s \\
= & e^{\mathbf{A}(t-\tau)} \boldsymbol{\Phi}_{\tau}+\int_{\tau}^{t} e^{\mathbf{A}(t-T)}\left(\mathbf{F}_{2}\left(\theta_{T} \omega\right)+\mathbf{F}_{3}(\boldsymbol{\Phi}(T-\tau))\right) d T
\end{aligned}
$$

Therefore,

$$
\begin{aligned}
& \boldsymbol{\Phi}^{A}\left(t-\tau, 0, \theta_{\tau} \omega ; \mathbf{\Phi}_{\tau}\right) \\
= & e^{\mathbf{A}(t-\tau)} \boldsymbol{\Phi}_{\tau}+\int_{\tau}^{t} e^{\mathbf{A}(t-T)}\left(\mathbf{F}_{2}\left(\theta_{T} \omega\right)+\mathbf{F}_{3}(\boldsymbol{\Phi})(T-\tau)\right) d T .
\end{aligned}
$$

Hence, $\boldsymbol{\Phi}^{A}\left(t-\tau, 0, \theta_{\tau} \omega ; \boldsymbol{\Phi}_{\tau}\right)$ is the value of a solution for Equation (10a) with initial value $t=\tau, \boldsymbol{\Phi}=\boldsymbol{\Phi}_{\tau}$ at time $t$. Then

$$
\boldsymbol{\Phi}^{A}\left(t-\tau, 0, \theta_{\tau} \omega ; \boldsymbol{\Phi}_{\tau}\right)=\boldsymbol{\Phi}^{A}\left(t, \tau, \omega ; \boldsymbol{\Phi}_{\tau}\right) .
$$

Furthermore, the solution mapping $\varphi^{A}(t, \tau, \omega)$ satisfies

$$
\varphi^{A}(t, \tau, \omega)=\varphi^{A}\left(t-\tau, 0, \theta_{\tau} \omega\right) .
$$

On the other hand, $\boldsymbol{\Phi}^{A}\left(\tau, \tau, \omega ; \boldsymbol{\Phi}_{\tau}\right)=\boldsymbol{\Phi}_{\tau}$ which means $\varphi^{A}(\tau, \tau, \omega)=0$. Thus, the proof is completedf. 


\subsection{Proof for Theorem 2}

It follows from Lemma 8 that the RDS $S(t, \omega)$ possesses global absorbing set in $E_{1}$. The estimation of expectation of radius of the global absorbing set is obtained in the proof for Lemma 8 if the condition that "for any nonrandom bounded set $B \subset E_{1}, \lim _{t \rightarrow+\infty} S^{A}\left(t, \theta_{-t} \omega\right) B$ is relative compact $\mathbb{P}$-a.s." can be satisfied, which is achieved in the following. Then, according the Theorem 5 , we can complete the proof of this theorem.

Suppose $\forall B \subset E_{1}$ is any bounded set, let $\widetilde{T}_{0} \geq T_{P}$ and

$$
\mathscr{A}_{n}^{B}(\omega)=\overline{\bigcup_{s \geq n} \widetilde{T}_{0}} S^{A}\left(s, \theta_{-s} \omega\right) B, n \in \mathbb{N}
$$

From Reference [67], we have

$$
\mathscr{A}_{n+1}^{B}(\omega) \subset \mathscr{A}_{n}^{B}(\omega), n \in \mathbb{N},
$$

and $\mathscr{A}_{n}^{B}(\omega), \forall n \in \mathbb{N}$ is nonempty closed set.

Let $\mathscr{A}_{\infty}^{B}(\omega)=\lim _{n \rightarrow \infty} \mathscr{A}_{n}^{B}(\omega), \mathcal{A}^{B}(\omega)=\lim _{T \rightarrow+\infty} S^{A}\left(T, \theta_{-T} \omega\right) B$, then

$$
\overline{\mathcal{A}}^{B}(\omega)=\overline{\mathscr{A}}_{\infty}^{B}(\omega)
$$

In the following, we verify $\mathcal{A}^{B}(\omega)$ is relative compact. It is enough to prove

$$
\lim _{T \rightarrow+\infty} \alpha_{K}\left(S^{A}\left(T, \theta_{-T} \omega\right) B\right)=0,
$$

where $\alpha_{K}(\cdot)$ denote the $\alpha$ - measure.

Taking advantage of the contradiction method, suppose Equation (70) is not true, then there exists $\widehat{\varepsilon}_{0}>0$, for $\forall \bar{T}_{1} \geq 0$ and $\bar{T}_{0}>\bar{T}_{1}$ such that

$$
\alpha_{K}\left(S^{A}\left(\bar{T}_{0}, \theta_{-\bar{T}_{0}} \omega\right) B\right) \geq 3 \widehat{\varepsilon}_{0} .
$$

Let $\bar{\varepsilon}_{0}=\widehat{\varepsilon}_{0}, \bar{T}_{1}=T_{B}(\bar{\varepsilon}, \omega), T_{0}=\bar{T}_{0}, T=\max \left\{T_{B}(\bar{\varepsilon}, \omega), T_{0}\right\}$, obviously, $T=\bar{T}_{0}$. Hence, $\alpha_{K}\left(S\left(\bar{T}_{0}, \theta_{-\bar{T}_{0}} \omega\right) B\right) \geq 3 \bar{\varepsilon}_{0}$ which reveals that there exists sequence $\left\{\mathbf{U}^{(0)}\right\}_{n=1}^{\infty} \in B$ such that

$$
\sup _{\tau \in \mathbb{R}}\left\|S^{A}\left(\bar{T}_{0}, \theta_{-\bar{T}_{0}} \omega\right) \mathbf{U}_{m}^{(0)}-S^{A}\left(\bar{T}_{0}, \theta_{-\bar{T}_{0}} \omega\right) \mathbf{U}_{n}^{(0)}\right\|_{E_{1}} \geq 2 \bar{\varepsilon}_{0}, m \neq n, n, m=1,2, \cdots
$$

which contradicts Equation (59). Thus, the supposed assumption is invalid which means Equation (70) holds. Then, $\alpha_{K}\left(\mathscr{A}_{\infty}^{B}(\omega)\right)=0$ which means $\lim _{n \rightarrow \infty} \alpha_{K}\left(\mathscr{A}_{n}^{B}(\omega)\right)=0$, together with (69), by the Proposition 3, we have $\bigcap_{n \geq 1} \mathscr{A}_{n}^{B}(\omega)$ is compact. On the other hand, since

$$
\bigcap_{n \geq 1} \mathscr{A}_{n}^{B}(\omega)=\bigcap_{N \geq 0} \overline{\bigcup_{n \geq N} S^{A}\left(n \widetilde{T}_{0}, \theta_{-n \widetilde{T}_{0}} \omega\right) B}
$$

Let

$$
\Lambda_{B}(\omega)=\bigcap_{N \geq 0} \overline{\bigcup_{n \geq N} S^{A}\left(n \widetilde{T}_{0}, \theta_{-n \widetilde{T}_{0}} \omega\right) B},
$$


then $\Lambda_{B}(\omega)$ is compact.

According to Theorem 5 , it can be found that the $\operatorname{RDS} S^{A}(t, \omega)$ possesses random attractors $\mathscr{A}(\omega) \subset E_{1}, \forall \omega \in \Omega$ defined by

$$
\mathscr{A}(\omega)=\overline{\bigcup_{B \subset E_{1}} \Lambda_{B}(\omega)}
$$

\subsection{Proof for Theorem 3}

By Equation (60), we have $\mathbf{F}_{2}\left(\mathbf{U}, \theta_{t} \omega\right)+\mathbf{F}_{3}(\mathbf{U}): E_{1} \rightarrow E_{1}$ satisfies Lipschtiz condition, and the rest of proof is very similar to the Proof for Theorem 1 provided in Section 4.4. Thus, it is omitted here.

\subsection{Proof for Theorem 4}

This process of proof can be completed with replacing Lemma 8 by Lemma 10, Equation (59) by Equation (68) and the $\operatorname{RDS} S^{A}(t, \omega)$ by $\operatorname{RDS} S^{M}(t, \omega)$ in the proof for Theorem 4 . Thus, it is omitted here.

\section{Summary and Conclusions}

The affirmation that expectation of radius of global random attractors for nonlinear stochastic plates considered in the paper is in direct proportion to the intensity of white noise and $F_{0}$ can be derived by Theorem 2 and Theorem 4 . On the other hand, it follows from Theorem 4 and the assumption in Equation (28) that, for the clamped Von Karman plate without rotational inertia driven by multiplicative white noise, values for the coefficient of the noise that are too large might result in the non-existence of global random attractor for the system, the phenomenon of which cannot be obtained in the status of additive white noise. The estimate of Equation (45) is derived in Section 3.2 to achieve the existence of global absorbing set for the system, which can also accomplished by Inequality (9.1.17) provided by Lemma 9.1.7 in Chueshov and Lasiecka [26]. However, the inequality in Equation (45) gives a clear relationship between $Y\left(\mathcal{F}, F_{0}\right)$ defined by Equation (44) in Section 3.2 and the in-plane force $F_{0}$, which along with Equation (46) indicates that $Y\left(\mathcal{F}, F_{0}\right)$ is determined by the component of the in-plane force on boundary along the direction $x, y$. This assertion can expounds the bucking phenomenon from the theoretical results on global random attractors.

Compared with the results on global attractors provided by Chen et al. [36] in situation of multiplicative noise, the investigation on existence of global random attractors for the systems, which are carried out in this paper, do not need the assumption that coefficient of the damping is big enough. This statement can be achieved by the process of proving the existence of global random absorbing set for clamped Von Karman plate without rotational inertia driven by white noise (see Lemma 8 in Section 3.2.1 and Lemma 10 in Section 3.2.2) and attaining the stabilization estimation of the systems(see Lemma 9 in Section 3.2.1 and Lemma 11 in Section 3.2.2). It is noticed that the condition for the existence of global random attractors presented by Chen et al. [36] is so conservative that, once there does not exist a steady ingredient of the loading, which means $p=0$ in the governing equations of Von Karman plate considered in [36], then the global random attractors only comprise the random fixed points (even the trivial solution). It is led by the too big coefficient of damping. The results obtained in this paper indicate that there exists global random attractors for the systems with the small coefficient of weak damping. Moreover, the statement that global random attractors are composed of more than a fixed random point is illustrated by the numerical results on global dynamics. On the other hand, the conclusion that multiplicative white noise is more likely to result in the appearance of global $\mathcal{D}$-bifurcation and secondary global $\mathcal{D}$-bifurcation in the motion of clamped Von Karman without rotational inertia than additive noises can also be validated from the numerical results on global dynamics of the systems.

In engineering applications, $\left(f_{1}, f_{2}\right)$ are the in-plane components of the external force that act on the edge of the plate. In light of Equations (3) and (4) given in Section 2.1, with Equation (46) attained 
in Section 3.2.1, the boundary value of $F_{0}$ and $\frac{\partial F_{0}}{\partial n}$ are determined by $\left(f_{1}, f_{2}\right)$. Thus, the aforementioned qualitative results on long time behavior for the two kinds of nonlinear stochastic Von Karman plates are demonstrated by the numerical results on global dynamics. Big enough value of external force that acts on the edge of the plate leads to the appearance of global $\mathcal{D}$-bifurcation in the motion of the simplified Von Karman plate without rotational inertia, which coincides with the phenomena named bucking [1]. Furthermore, the fact mentioned above together with assertion that global $\mathcal{D}$-bifurcation would disappear with increasing the value of intensity of the white noise can give a reasonable explanation of how the random snap-through [68] occurs in motion of the stochastic Von Karman plate and how to eliminate it in the following manner. When the global random attractor only comprise a fixed random point, then random snap-through occurs. Once the global $\mathcal{D}$-bifurcation appears in the motion of the Von Karman plate, the random snap-through will disappears. Alternatively, the large value of the intensity of the white noise results in the occurring of the random snap-through which can be eliminated by increasing the value of external force that act on the edge of the plate. As indicated above, it can be concluded that the random snap-through which seems to be a complex dynamics intuitively is essentially a simple dynamical behavior.

Author Contributions: Conceptualization, Investigation, Writing-Original Draft Preparation, and Writing-Review and Editing, H.C.; Investigation, Supervision, Project Administration, Funding Acquisition, and Writing-Review and Editing, D.C.; Investigation, Writing-Review and Editing, J.J.; Resources; and Writing-Review and Editing, X.F.

Funding: This work was supported by the Key Project of National Natural Science Foundation of China (No. 11732005) and the National Natural Science Foundation of China (No. 91216106, 11472089).

Conflicts of Interest: The authors declare no conflict of interest.

\section{Abbreviations}

The following abbreviations are used in this manuscript:

RDS Random Dynamical System

SAVKP The system described by Equations (10a), (10c), (10e)-(10h)

SAVKPT1 The system described by Equations (10c), (15) and (10e)-(10h)

SAVAPT2 The system described by Equations (18), (10c) and (10e)-(10h)

SMVKP The system interpreted by Equations (10b), (10c), (10e)-(10h)

SMVKPT1 The system interpreted by Equations (20), (10c) and (10e)-(10f)

SMVAPT2 The system interpreted by Equations (21), (10c) and (10e)-(10f)

\section{Appendix A. The Model Equation}

Suppose $u=\sum_{i=1}^{s} l_{i}(t) w_{i}, \mathcal{F}=\sum_{i=1}^{s_{0}} a_{i}(t) w_{i}, s=s_{1}+s_{2}, s_{0}, s_{1}, s_{2} \in \mathbb{N}$, where $\left\{w_{i}\right\}_{i=1}^{4}$ are eigenvectors related to eigenvalues $\left\{\Lambda_{i}\right\}_{i=1}^{k}$ of operator $A$. Taking the inner product of Equation (10e) by $w_{i}, i=$ $1, \cdots, k$ in $L^{2}$, we have

$$
a_{i}(t)=-\frac{1}{\Lambda_{i}}\left(\left[\sum_{i=1}^{s} l_{i}(t) w_{i}, \sum_{i=1}^{s} l_{i}(t) w_{i}\right], w_{i}\right), i=1, \cdots, s_{0},
$$

then

$$
\mathcal{F}=\sum_{i=1}^{s_{0}}-\frac{1}{\Lambda_{i}}\left(\left[\sum_{i=1}^{s} l_{i}(t) w_{i}, \sum_{i=1}^{s} l_{i}(t) w_{i}\right], w_{i}\right) w_{i} .
$$

Thus, the model equations associated with SAVKP have the following form

$$
\begin{aligned}
& d \underline{\mathbf{m}}=\left(\underline{\mathbf{M}}_{1} \underline{\mathbf{m}}+\underline{\mathbf{M}}_{2}(\underline{\mathbf{m}}, \overline{\mathbf{m}})\right) d t+\sigma \underline{\mathbf{M}}_{3} d W, \\
& d \overline{\mathbf{m}}=\left(\overline{\mathbf{M}}_{2} \overline{\mathbf{m}}+\overline{\mathbf{M}}_{2}(\underline{\mathbf{m}}, \overline{\mathbf{m}})\right) d t+\sigma \overline{\mathbf{M}}_{3} d W, t \in(\tau h-h, \tau h] ; \\
& \overline{\mathbf{m}}=\overline{\mathbf{m}}_{\tau h-h}, t \notin(\tau h-h, \tau h]
\end{aligned}
$$


where $\underline{1}=\left(\underline{l}_{1}, \cdots, \underline{l}_{k_{1}}\right)^{T}=\left(l_{1}, \cdots, l_{k_{1}}\right)^{T}$ represents the low-frequency modal and the other is high-frequency modal $\overline{\mathbf{1}}=\left(\bar{l}_{1}, \cdots, \bar{l}_{k_{2}}\right)^{T}=\left(l_{k_{1}}, \cdots, l_{k_{1}+k_{2}}\right)^{T}, \mathbf{1}_{i}$ is the value of $\mathbf{1}$ at time $i . \underline{\mathbf{g}}=\underline{\mathbf{l}}_{t}, \overline{\mathbf{g}}=$ $\overline{\mathbf{1}}_{t}, \underline{\mathbf{m}}=\left[\underline{l}_{1}, \underline{g}_{1}, \cdots, \underline{l}_{k}, \underline{g}_{k_{1}}\right]^{T}, \overline{\mathbf{m}}=\left[\bar{l}_{1}, \bar{g}_{1}, \ldots, \bar{l}_{k}, \bar{g}_{k_{2}}\right]^{T} . h$ is step size of numerical integration. $\tau \in \mathbb{N}$ is an undetermined constant.

$$
\begin{gathered}
\underline{\mathbf{M}}_{1}=\left[\begin{array}{ccccc}
0 & 1 & \mathbf{0} & 0 & 0 \\
-\Lambda_{1} & -\alpha & \mathbf{0} & 0 & 0 \\
\mathbf{0} & \mathbf{0} & \ddots & \mathbf{0} & \mathbf{0} \\
0 & 0 & \mathbf{0} & -\Lambda_{s_{1}} & -\alpha
\end{array}\right], \\
0 \\
\underline{\mathbf{M}}_{2}(\underline{\mathbf{m}}, \overline{\mathbf{m}})=-\left[\begin{array}{c}
0 \\
F_{1}(\mathbf{1}) \\
\vdots \\
0 \\
F_{s_{1}}(\mathbf{1})
\end{array}\right], \underline{\mathbf{M}}_{3}=\left[\begin{array}{c}
\left(q(x), w_{1}\right) \\
\vdots \\
0 \\
\left(q(x), w_{s_{1}}\right)
\end{array}\right]
\end{gathered}
$$

and

in which

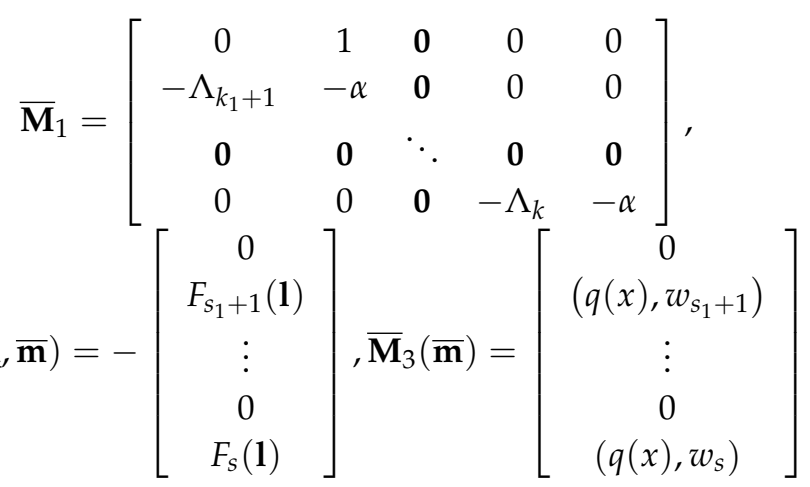

$$
\begin{gathered}
\overline{\mathbf{F}}=\left[\begin{array}{c}
\bar{F}_{1} \\
\vdots \\
\bar{F}_{s}
\end{array}\right]=\left[\begin{array}{c}
\left(\sigma q(x) \dot{W}, w_{1}\right) \\
\vdots \\
\left(\sigma q(x) \dot{W}, w_{s}\right)
\end{array}\right] \\
\mathbf{F}(\mathbf{1})=\left[\begin{array}{c}
F_{1}(\mathbf{1}) \\
\vdots \\
F_{2}(\mathbf{1})
\end{array}\right]=k\left[\begin{array}{c}
-\left(\left[\mathcal{F}, \sum_{i=1}^{s} l_{i} w_{i}\right], w_{1}\right)-\left(\left[F_{0}, \sum_{i=1}^{s} l_{i} w_{i}\right], w_{1}\right) \\
\vdots \\
-\left(\left[\mathcal{F}, \sum_{i=1}^{s} l_{i} w_{i}\right], w_{s}\right)-\left(\left[F_{0}, \sum_{i=1}^{s} l_{i} w_{i}\right], w_{s}\right)
\end{array}\right],
\end{gathered}
$$

Furthermore, let

$$
\widehat{\mathbf{M}}_{3}=\left[\begin{array}{c}
0 \\
\underline{l}_{1} \\
\vdots \\
0 \\
\underline{l}_{s_{1}}
\end{array}\right], \widehat{\mathbf{M}}_{3}=\left[\begin{array}{c}
0 \\
\bar{l}_{1} \\
\vdots \\
0 \\
\bar{l}_{s_{2}}
\end{array}\right],
$$

then, respectively, replacing $\underline{\mathbf{M}}_{3}, \overline{\mathbf{M}}_{3}$ in Equation (A1) by $\widehat{\mathbf{M}}_{3}, \widehat{\mathbf{M}}_{3}$ leads to the modal equations for SMVKP. 


\section{Appendix B. Proof of the Lemmas in Section 3}

Appendix B.1. Proof of Lemma 2

Since

$$
\begin{aligned}
\int_{D} \mathcal{F} \Delta^{2} F_{0} d x & =\int_{\Gamma} \frac{\partial \Delta F_{0}}{\partial n} \mathcal{F}-\int_{D} \nabla\left(\Delta F_{0}\right) \cdot \nabla \mathcal{F} d x \\
& =\int_{\Gamma} \frac{\partial \Delta F_{0}}{\partial n} \mathcal{F} d s-\int_{\Gamma} \frac{\partial \mathcal{F}}{\partial n} \Delta F_{0} d s+\int_{D} \Delta \mathcal{F} \Delta F_{0} d x
\end{aligned}
$$

together with Equation (10f) gives

$$
\int_{D} \mathcal{F} \Delta^{2} F_{0} d x=\int_{D} \Delta \mathcal{F} \Delta F_{0} d x
$$

Taking into account Equation (10g), we have

$$
\int_{D} \Delta \mathcal{F} \Delta F_{0} d x=0
$$

Appendix B.2. Proof of Lemma 6

Let $\bar{E}=\left(H^{4} \times L^{2}\right)(D)$, define

$$
a(\mathbf{U}, \mathbf{V})=\left\langle-\mathbf{A}_{1} \mathbf{U}, \mathbf{V}\right\rangle,
$$

where $<\square, \square>=\int_{D} \square \cdot \square d x$. By Cauchy inequality, there exists $M \geq 0$ such that

$$
\begin{aligned}
& \left|<-\mathbf{A}_{1} \mathbf{U}, \mathbf{V}>\right| \\
= & \mid\left(-\varepsilon U_{1}+U_{2}, U_{1}\right)+\left(-A U_{1}+\varepsilon(\alpha-\varepsilon) U_{1}, U_{2}\right) \\
& -\left((\alpha-\varepsilon) U_{2}, U_{2}\right) \mid, \\
\leq & M\|\mathbf{U}\|_{\bar{E}}\|\mathbf{V}\|_{\bar{E}} . \forall \mathbf{U}, \mathbf{V} \in \bar{E}
\end{aligned}
$$

From the Proofs of Lax-Milgram given by Temam [55], we have

$$
-\mathbf{A}_{1} \in \mathscr{L}\left(\bar{E}, \bar{E}^{*}\right),
$$

where $\bar{E}^{*}$ is the conjugate space of $\bar{E}$. Moreover, Equation (49) yields

$$
\left(-\mathbf{A}_{1} \mathbf{U}, \mathbf{U}\right)_{E_{1}} \geq \frac{\varepsilon}{2}\|\mathbf{U}\|_{E_{1}}^{2}, \forall \mathbf{U} \in E_{2} .
$$

Let

$$
\mathcal{D}_{0}=\left\{\mathbf{U} \mid \mathbf{U} \in \bar{E},-\mathbf{A}_{1} \mathbf{U} \in E_{1}\right\},
$$

then $\mathcal{D}_{0}=E_{2}$. Since $E_{1} \subset \bar{E}^{*}$, then (A5) yields $-\mathbf{A}_{1} \in \mathscr{L}\left(E_{2}, E_{1}\right)$.

Furthermore, Invoking Equation (A6), this gives $-\mathbf{A}_{1}: E_{2} \rightarrow E_{1}$ is injection. On the other hand, since $E_{1} \subset \bar{E}^{*}$, by applying (A5), we have $-\mathbf{A}_{1}: E_{2} \rightarrow E_{1}$ is surjection. By open mapping theorem, we obtain that the inverse of $-\mathbf{A}_{1}$ exists and $\left(-\mathbf{A}_{1}\right)^{-1} \in \mathscr{L}\left(E_{1}, E_{1}\right)$. 
Appendix B.3. Proof of Lemma 7

Without loss of generality, suppose $\left[w_{k}, e_{k}\right]^{T}, k \in \mathbb{N}$ is eigenvector with respect to eigenvalues $\lambda_{k}$, then

$$
\mathbf{A}_{1}\left[w_{k}, e_{k}\right]^{T}=\lambda_{k}\left[w_{k}, e_{k}\right]^{T}
$$

Thus,

$$
\begin{aligned}
-\varepsilon w_{k}+e_{k} & =\lambda_{k} w_{k \prime} \\
-A w_{k}+\varepsilon(\alpha-\varepsilon) w_{k} & -(\alpha-\varepsilon) e_{k}=\lambda_{k} e_{k} .
\end{aligned}
$$

Equation (A8) yields $e_{k}=\left(\lambda_{k}+\varepsilon\right) w_{k}$; substituting it into Equation (A9), we have

$$
-A w_{k}+\varepsilon(\alpha-\varepsilon) w_{k}-(\alpha-\varepsilon)\left(l_{k}+\varepsilon\right) w_{k}=\lambda_{k}\left(\lambda_{k}+\varepsilon\right) w_{k}
$$

then

$$
-A w_{k}-\alpha \lambda_{k} w_{k}=\lambda_{k}^{2} w_{k}
$$

Since $A w_{k}=\Lambda_{k} w_{k}$, we obtain

$$
-\Lambda_{k} w_{k}-\alpha \lambda_{k} w_{k}=\lambda_{k}^{2} w_{k}
$$

Hence,

$$
\lambda_{k}^{(+)}=\frac{-\alpha+\sqrt{\alpha^{2}-4 \Lambda_{k}}}{2}, \lambda_{k}^{(-)}=\frac{-\alpha-\sqrt{\alpha^{2}-4 \Lambda_{k}}}{2} .
$$

Appendix B.4. Proof of Lemma 8

Taking the inner product of Equation (18) by $\boldsymbol{\Phi}$ in $E_{1}$ gives

$$
\begin{aligned}
& \frac{d}{d t}\|\boldsymbol{\Phi}\|_{E_{1}}^{2} \\
= & 2\left(\mathbf{A}_{1} \boldsymbol{\Phi}, \boldsymbol{\Phi}\right)_{E_{1}}+2\left(\sigma A^{\frac{1}{2}} q(x) z\left(\theta_{t} \omega\right), A^{\frac{1}{2}} \Phi_{1}\right) \\
& 2\left(\sigma(\mu-\alpha+\varepsilon) q(x) z\left(\theta_{t} \omega\right), \Phi_{2}\right)+2 k\left(\left[\mathcal{F}\left(\Phi_{1}\right)+F_{0}, \Phi_{1}\right], \Phi_{2}\right) .
\end{aligned}
$$

According to Lemma 5, we find

$$
2\left(\mathbf{A}_{1} \boldsymbol{\Phi}, \boldsymbol{\Phi}\right)_{E_{1}} \leq-\varepsilon\|\boldsymbol{\Phi}\|_{E_{1}}^{2}-k_{0}\left\|\Phi_{2}\right\|,
$$

It follows from Equations (57) and (58) that the third and fourth term on the right of the inequality sign in Equation (A11) can be controlled by

$$
\frac{\varepsilon}{4}\left\|A^{\frac{1}{2}} \Phi_{1}\right\|^{2}+\frac{k_{0}}{2}\left\|\Phi_{2}\right\|^{2}+\frac{4 \sigma^{2}\left|z\left(\theta_{t} \omega\right)\right|^{2}}{\varepsilon}\left\|A^{\frac{1}{2}} q(x)\right\|^{2}+\frac{\left(\sigma(\mu-\alpha+\varepsilon) q(x) z\left(\theta_{t} \omega\right)\right)^{2}}{k_{0}}\|q(x)\|^{2},
$$

Since $\boldsymbol{\Phi}=\left[\Phi_{1}, \Phi_{2}\right]^{T}=[u, v]^{T}$, utilizing Equations (55) and (56), we have

$$
\begin{aligned}
& 2 k\left(\left[\mathcal{F}\left(\Phi_{1}\right)+F_{0}, \Phi_{1}\right], \Phi_{2}\right) \\
\leq & -\frac{k}{2} \frac{d}{d t}\left\|\Delta \mathcal{F}\left(\Phi_{1}\right)\right\|^{2}-2 k \varepsilon\left\|\Delta \mathcal{F}\left(\Phi_{1}\right)\right\|^{2}+2 \sqrt{\bar{k}}\left|\sigma z\left(\theta_{t} \omega\right)\right|\|q\|\left(\left\|A^{\frac{1}{2}} \Phi_{1}\right\|^{2}+\frac{k}{4}\left\|\Delta \mathcal{F}\left(\Phi_{1}\right)\right\|^{2}\right) \\
& -k Y\left(\mathcal{F}, F_{0}\right)-2 k \varepsilon Y\left(\mathcal{F}, F_{0}\right)+\frac{\varepsilon}{4}\left\|A \Phi_{1}\right\|^{2}+\frac{4 k^{2}}{\varepsilon}\left(\left|\sigma z\left(\theta_{t} \omega\right)\right|\|q\|_{1}\left\|F_{0}\right\|_{2}\right)^{2} .
\end{aligned}
$$


as indicated above, we get

$$
\begin{aligned}
\frac{d}{d t}\|\boldsymbol{\Phi}\|_{E_{1}}^{2} \leq & -\frac{3 \varepsilon}{4}\|\boldsymbol{\Phi}\|_{E_{1}}^{2}+\frac{4 \sigma^{2}\left|z\left(\theta_{t} \omega\right)\right|^{2}}{\varepsilon}\left\|A^{\frac{1}{2}} q(x)\right\|^{2}+\frac{\left(\sigma(\mu-\alpha+\varepsilon) q(x) z\left(\theta_{t} \omega\right)\right)^{2}}{k_{0}}\|q(x)\|^{2} \\
& -\frac{k}{2} \frac{d}{d t}\left\|\Delta \mathcal{F}\left(\Phi_{1}\right)\right\|^{2}-2 k \varepsilon\left\|\Delta \mathcal{F}\left(\Phi_{1}\right)\right\|^{2}+2 \sqrt{\bar{k}}\left|\sigma z\left(\theta_{t} \omega\right)\right|\|q\|\left(\left\|A^{\frac{1}{2}} \Phi_{1}\right\|^{2}+\frac{k}{4}\left\|\Delta \mathcal{F}\left(\Phi_{1}\right)\right\|^{2}\right) \\
& -k \frac{d}{d t} \mathrm{Y}\left(\mathcal{F}, F_{0}\right)-2 k \varepsilon Y\left(\mathcal{F}, F_{0}\right)+\frac{\varepsilon}{4}\left\|A \Phi_{1}\right\|^{2}+\frac{4 k^{2}}{\varepsilon}\left(\mid \sigma z\left(\theta_{t} \omega\right)\|q\|_{1}\left\|F_{0}\right\|_{2}\right)^{2} \\
\leq & -\frac{\varepsilon}{2}\|\boldsymbol{\Phi}\|_{E_{1}}^{2}-\frac{k}{2} \frac{d}{d t}\left\|\Delta \mathcal{F}\left(\Phi_{1}\right)\right\|^{2}-k \frac{d}{d t} \mathrm{Y}\left(\mathcal{F}, F_{0}\right)-\frac{k \varepsilon}{4}\left\|\Delta \mathcal{F}\left(\Phi_{1}\right)\right\|^{2}-\frac{k \varepsilon}{2} \mathrm{Y}\left(\mathcal{F}, F_{0}\right) \\
& +2 \sqrt{\bar{k}} \mid \sigma z\left(\theta_{t} \omega\right)\|q\|\left(\left\|A^{\frac{1}{2}} \Phi_{1}\right\|^{2}+\frac{k}{4}\left\|\Delta \mathcal{F}\left(\Phi_{1}\right)\right\|^{2}\right)+\frac{4 k^{2}}{\varepsilon}\left(\left|\sigma z\left(\theta_{t} \omega\right)\right|\|q\|_{1}\left\|F_{0}\right\|_{2}\right)^{2} \\
& +\frac{4 \sigma^{2}\left|z\left(\theta_{t} \omega\right)\right|^{2}}{\varepsilon}\left\|A^{\frac{1}{2}} q(x)\right\|^{2}+\frac{\left(\sigma(\mu-\alpha+\varepsilon) q(x) z\left(\theta_{t} \omega\right)\right)^{2}}{k_{0}}\|q(x)\|^{2} \\
& -\frac{7 k \varepsilon}{4} \varepsilon\left\|\Delta \mathcal{F}\left(\Phi_{1}\right)\right\|^{2}-\frac{3 k \varepsilon}{2}\left|\mathrm{Y}\left(\mathcal{F}, F_{0}\right)\right| .
\end{aligned}
$$

By means of Equation (52), we catch

$$
\begin{aligned}
\frac{d}{d t}\|\boldsymbol{\Phi}\|_{E_{1}}^{2} \leq & -\frac{k}{2} \frac{d}{d t}\left\|\Delta \mathcal{F}\left(\Phi_{1}\right)\right\|^{2}-k \frac{d}{d t} Y\left(\mathcal{F}, F_{0}\right)-\frac{\varepsilon}{2}\|\boldsymbol{\Phi}\|_{E_{1}}^{2}-\frac{k \varepsilon}{4} \varepsilon\left\|\Delta \mathcal{F}\left(\Phi_{1}\right)\right\|^{2}-\frac{k \varepsilon}{2} Y\left(\mathcal{F}, F_{0}\right) \\
& +2 \sqrt{\bar{k}} \mid \sigma z\left(\theta_{t} \omega\right)\|q\|\left(\left\|A^{\frac{1}{2}} \Phi_{1}\right\|^{2}+\frac{k}{4}\left\|\Delta \mathcal{F}\left(\Phi_{1}\right)\right\|^{2}\right) \\
& +\frac{3 k \varepsilon}{2} C\left(\frac{7}{6}, C_{1}, C_{2}\right)\left\|F_{0}\right\|_{2}^{2}+\frac{\left(\sigma(\mu-\alpha+\varepsilon) q(x) z\left(\theta_{t} \omega\right)\right)^{2}}{k_{0}}\|q(x)\|^{2} \\
& +\frac{4 \sigma^{2}\left|z\left(\theta_{t} \omega\right)\right|^{2}}{\varepsilon}\left\|A^{\frac{1}{2}} q(x)\right\|^{2}+\frac{4 k^{2}}{\varepsilon}\left(\mid \sigma z\left(\theta_{t} \omega\right)\|q\|_{1}\left\|F_{0}\right\|_{2}\right)^{2}
\end{aligned}
$$

It can be derived from Equation (54) that

$$
\begin{aligned}
\frac{d}{d t}\|\boldsymbol{\Phi}\|_{E_{1}}^{2} \leq & -\frac{k}{2} \frac{d}{d t}\left\|\Delta \mathcal{F}\left(\Phi_{1}\right)\right\|^{2}-k \frac{d}{d t} Y\left(\mathcal{F}, F_{0}\right)-\frac{\varepsilon}{2}\|\boldsymbol{\Phi}\|_{E_{1}}^{2}-\frac{k \varepsilon}{4} \varepsilon\left\|\Delta \mathcal{F}\left(\Phi_{1}\right)\right\|^{2}-\frac{k \varepsilon}{2} Y\left(\mathcal{F}, F_{0}\right) \\
& +2 \sqrt{\bar{k}}\left|\sigma z\left(\theta_{t} \omega\right)\right|\|q\|\left(\left\|A^{\frac{1}{2}} \Phi_{1}\right\|^{2}+\frac{k}{4}\left\|\Delta \mathcal{F}\left(\Phi_{1}\right)\right\|^{2}+k Y\left(\mathcal{F}, F_{0}\right)\right) \\
& +\frac{\left(\sigma(\mu-\alpha+\varepsilon) q(x) z\left(\theta_{t} \omega\right)\right)^{2}}{k_{0}}\|q(x)\|^{2}+2 \sqrt{\bar{k}} k\left|\sigma z\left(\theta_{t} \omega\right)\right|\|q\| C\left(\frac{1}{4}, C_{1}, C_{2}\right)\left\|F_{0}\right\|_{2}^{2} \\
& +\frac{4 \sigma^{2}\left|z\left(\theta_{t} \omega\right)\right|^{2}}{\varepsilon}\left\|A^{\frac{1}{2}} q(x)\right\|^{2}+\frac{4 k^{2}}{\varepsilon}\left(\left|\sigma z\left(\theta_{t} \omega\right)\right|\|q\|_{1}\left\|F_{0}\right\|_{2}\right)^{2} \\
& +\frac{3 k \varepsilon}{2} C\left(\frac{7}{6}, C_{1}, C_{2}\right)\left\|F_{0}\right\|_{2}^{2} .
\end{aligned}
$$

Let

$$
\begin{gathered}
\mathcal{E}_{1}(t, \tau, \omega)=\overline{\mathcal{E}}_{1}\left(\Phi_{1}, \Phi_{2}, \omega\right)=\left(\left\|\Phi_{2}\right\|^{2}+\left\|A^{\frac{1}{2}} \Phi_{1}\right\|^{2}+\frac{k}{2}\left\|\Delta \mathcal{F}\left(\Phi_{1}\right)\right\|^{2}+k Y\left(\mathcal{F}, F_{0}\right)\right) \\
\mathcal{E}(t, \tau, \omega)=\overline{\mathcal{E}}\left(\Phi_{1}, \Phi_{2}, \omega\right)=\left(\left\|\Phi_{2}\right\|^{2}+\left\|A^{\frac{1}{2}} \Phi_{1}\right\|^{2}+\frac{k}{2}\left\|\Delta \mathcal{F}\left(\Phi_{1}\right)\right\|^{2}\right)
\end{gathered}
$$

Invoking Equation (53), we have

$$
\mathcal{E}\left(\Phi_{1}, \Phi_{2}, \omega\right) \leq \mathcal{E}_{1}\left(\Phi_{1}, \Phi_{2}, \omega\right)+k C\left(\frac{1}{2}, C_{1}, C_{2}\right)\left\|F_{0}\right\|_{2}^{2}
$$


which along with Equations (25) and (A11) gives

$$
\frac{d}{d t}\left\|\overline{\mathcal{E}}_{1}\left(\Phi_{1}, \Phi_{2}, \omega\right)\right\|_{E_{1}}^{2} \leq-\frac{\varepsilon}{2} \overline{\mathcal{E}}_{1}\left(\Phi_{1}, \Phi_{2}, \omega\right)+2 \sqrt{\bar{k}} \mid \sigma z\left(\theta_{t} \omega\right)\|q\| \overline{\mathcal{E}}_{1}\left(\Phi_{1}, \Phi_{2}, \omega\right)+\bar{K}
$$

Setting $\epsilon=\frac{\varepsilon}{2}$ and then employing Equation (A13), it can be asserted that, for any give $\tau \in \mathbb{R}, \forall t \geq$ $\tau$, the next holds

$$
\mathcal{E}_{1}(t, \tau, \omega) \leq e^{-\epsilon(t-\tau)+\int_{\tau}^{t} 2 \sqrt{\bar{k}}\left|\sigma z\left(\theta_{s} \omega\right)\right|\|q\| d s} \mathcal{E}_{1}(\tau, \tau, \omega)+\int_{\tau}^{t} e^{-\epsilon(t-s)+\int_{s}^{t} 2 \sqrt{\bar{k}} \mid \sigma z\left(\theta_{s} \omega\right)\|q\| d k} K d s .
$$

which means that for any $t<0$,

$$
\mathcal{E}_{1}(0, t, \omega) \leq e^{\epsilon t+\int_{t}^{0} 2 \sqrt{\bar{k}}\left|\sigma z\left(\theta_{s} \omega\right)\right|\|q\| d s} \mathcal{E}_{1}(t, t, \omega)+\int_{t}^{0} e^{\epsilon t+\int_{t}^{0} 2 \sqrt{\bar{k}} \mid \sigma z\left(\theta_{s} \omega\right)\|q\| d k} K d s
$$

Incorporating Equation (A12), we obtain

$$
\begin{aligned}
\mathcal{E}(0, t, \omega) \leq & e^{\epsilon t+\int_{t}^{0} 2 \sqrt{\bar{k}}\left|\sigma z\left(\theta_{s} \omega\right)\right|\|q\| d s} \mathcal{E}_{1}(t, t, \omega)+\int_{t}^{0} e^{\epsilon t+\int_{t}^{0} 2 \sqrt{\bar{k}} \mid \sigma z\left(\theta_{s} \omega\right)\|q\| d k} K d s \\
& +k C\left(\frac{1}{2}, C_{1}, C_{2}\right)\left\|F_{0}\right\|_{2}^{2} .
\end{aligned}
$$

Since $\boldsymbol{\Phi}=\left[\Phi_{1}, \Phi_{2}\right]^{T}=[u, v]^{T}, v=u_{t}+\varepsilon u-\sigma q(x) z\left(\theta_{t} \omega\right)$, then

$$
\begin{aligned}
\left(\left\|A^{\frac{1}{2}} u\right\|^{2}+\left\|u_{t}+\varepsilon u\right\|^{2}\right)(0, t, \omega) \leq & e^{\epsilon t+\int_{t}^{0} 2 \sqrt{\bar{k}} \mid \sigma z\left(\theta_{s} \omega\right)\|q\| d s} \mathcal{E}(t, t, \omega) \\
& +\int_{t}^{0} e^{\epsilon t+\int_{t}^{0} 2 \sqrt{\bar{k}}\left|\sigma z\left(\theta_{s} \omega\right)\right|\|q\| d k} K d s \\
& +e^{\epsilon t+\int_{t}^{0} 2 \sqrt{\bar{k}}\left|\sigma z\left(\theta_{s} \omega\right)\right|\|q\| d s} \sigma^{2}\|q(x)\|^{2}\left|z\left(\theta_{t} \omega\right)\right|^{2} \\
& +\sigma^{2}\|q(x)\|^{2}|z(\omega)|^{2}
\end{aligned}
$$

By Equation (34), we have that there exists $T_{1}<0$, such that for $\forall t<T_{1}$, the following holds( $(\mathbb{P}$-a.s.)

$$
-\frac{1}{t} \int_{0}^{t} 2 \sqrt{\bar{k}}\left|\sigma z\left(\theta_{s} \omega\right)\right|\|q\| d s \geq-\frac{4 \sqrt{\bar{k}}|\sigma|\|q\|}{\sqrt{\pi \mu}}
$$

furthermore, according to Equation (24), we find $\epsilon_{0} \equiv \epsilon-\frac{4 \sqrt{\bar{k}}|\sigma|\|q\|}{\sqrt{\pi \mu}}>0$, then

$$
\left(\epsilon-\frac{1}{t} \int_{0}^{t} 2 \sqrt{\bar{k}}\left|\sigma z\left(\theta_{s} \omega\right)\right|\|q\| d s\right) t \leq \epsilon_{0} t, \quad \forall t<T_{1} .
$$

Since $z(\omega)$ is tempered, we have

$$
e^{\epsilon t+\int_{t}^{0} 2 \sqrt{\bar{k}}\left|\sigma z\left(\theta_{s} \omega\right)\right|\|q\| d s} \mathcal{E}(t, t, \omega) \rightarrow 0, t \rightarrow-\infty,
$$

and

$$
e^{\epsilon t+\int_{t}^{0} 2 \sqrt{\bar{k}}\left|\sigma z\left(\theta_{s} \omega\right)\right|\|q\| d s} \sigma^{2}\|q(x)\|^{2}\left|z\left(\theta_{t} \omega\right)\right|^{2} \rightarrow 0, t \rightarrow-\infty
$$


Merging with Equation (25), we attain

$$
\begin{aligned}
& \int_{-\infty}^{0} e^{\epsilon t+\int_{t}^{0} 2 \sqrt{\bar{k}}\left|\sigma z\left(\theta_{s} \omega\right)\right|\|q\| d k} K d s \\
\leq & \int_{T_{1}}^{0} e^{\epsilon s+\int_{s}^{0} 2 \sqrt{\bar{k}}\left|\sigma z\left(\theta_{s} \omega\right)\right|\|q\| d k} K_{0} d s+\int_{-\infty}^{T_{1}} e^{\epsilon_{0} s} K_{0} d s \\
& +\int_{T_{1}}^{0} e^{\epsilon s+\int_{s}^{0} 2 \sqrt{\bar{k}}\left|\sigma z\left(\theta_{s} \omega\right)\right|\|q\| d k}\left|z\left(\theta_{s} \omega\right)\right| K_{1} d s+\int_{-\infty}^{T_{1}} e^{\epsilon_{0} s}\left|z\left(\theta_{s} \omega\right)\right| K_{1} d s . \\
& +\int_{T_{1}}^{0} e^{\epsilon s+\int_{s}^{0} 2 \sqrt{\bar{k}}\left|\sigma z\left(\theta_{s} \omega\right)\right|\|q\| d k}\left|z\left(\theta_{s} \omega\right)\right|^{2} K_{2} d s+\int_{-\infty}^{T_{1}} e^{\epsilon_{0} s}\left|z\left(\theta_{s} \omega\right)\right|^{2} K_{2} d s .
\end{aligned}
$$

then $\rho(\omega)$ defined by Equation (26) is bounded( $\mathbb{P}$-a.s.).

It follows from Equations (A15) to (A18) that there exists $\overline{T_{B}}(\omega)<0$, such that, for $\forall \bar{t} \leq \overline{T_{B}}(\omega)$, the following holds

$$
\left(\left\|A^{\frac{1}{2}} u\right\|^{2}+\left\|u_{t}+\varepsilon u\right\|^{2}\right)(0, \bar{t}, \omega) \leq \rho(\omega) .
$$

On the other hand, since

$$
\left(\left\|A^{\frac{1}{2}} u\right\|^{2}+\left\|u_{t}+\varepsilon u\right\|^{2}\right)(0, \bar{t}, \omega)=\left(\left\|A^{\frac{1}{2}} u\right\|^{2}+\left\|u_{t}+\varepsilon u\right\|^{2}\right)\left(-\bar{t}, \theta_{\bar{t}} \omega\right),
$$

let $t=-\bar{t}, T_{B}(\omega)=-\overline{T_{B}}(\omega)$, then, for any non-random bounded set $B \subset E_{1}$, there exists $T_{B}(\omega)>0$, such that, for $\forall t>T_{B}(\omega)$, the following holds ( $\mathbb{P}$-a.s.)

$$
\left\|S^{A}\left(t, \theta_{-t} \omega\right) B\right\|_{E_{1}} \leq r(\omega) .
$$

The rest is intended to estimate the expectation of $\rho(\omega)$. Invoking Equation (36), we have

$$
\mathbb{E}\left(e^{\epsilon S+\int_{s}^{0} 2 \sqrt{\bar{k}}\left|\sigma z\left(\theta_{k} \omega\right)\right|\|q\| d k}\right) \leq e^{\epsilon S-\frac{2 \sqrt{\bar{k}}|\sigma|\|q\|}{\sqrt{\mu}} S},
$$

on the other hand,

$$
\left(\mathbb{E}\left(\left|z\left(\theta_{s} \omega\right)\right|^{4}\right)\right)^{\frac{1}{2}}=\left(\frac{\Gamma\left(\frac{5}{2}\right)}{\mu^{2} \sqrt{\pi}}\right)^{\frac{1}{2}},\left(\mathbb{E}\left(\left|z\left(\theta_{s} \omega\right)\right|^{2}\right)\right)^{\frac{1}{2}}=\left(\frac{\Gamma\left(\frac{3}{2}\right)}{\mu^{2} \sqrt{\pi}}\right)^{\frac{1}{2}},
$$

where $\Gamma(\cdot)$ is $\Gamma$ function, which together with Equation (A21) and Cauchy equality gives

$$
\begin{aligned}
& \mathbb{E}\left(e^{\epsilon S+\int_{s}^{0} 2 \sqrt{k}\left|\sigma z\left(\theta_{k} \omega\right)\right|\|q\| d k}\left|z\left(\theta_{S} \omega\right)\right|^{2}\right) \leq e^{\epsilon S-\frac{2 \sqrt{k}|\sigma|\|q\|}{\sqrt{\mu}} s}\left(\frac{\Gamma\left(\frac{5}{2}\right)}{\mu^{2} \sqrt{\pi}}\right)^{\frac{1}{2}}, \\
& \mathbb{E}\left(e^{\epsilon S+\int_{s}^{0} 2 \sqrt{k}\left|\sigma z\left(\theta_{k} \omega\right)\right|\|q\| d k}\left|z\left(\theta_{S} \omega\right)\right|\right) \leq e^{\epsilon S-\frac{2 \sqrt{k}|\sigma|\|||\|_{s}}{\sqrt{\mu}}}\left(\frac{\Gamma\left(\frac{3}{2}\right)}{\mu^{2} \sqrt{\pi}}\right)^{\frac{1}{2}} .
\end{aligned}
$$

Define

$$
M=M_{0}+M_{1}+M_{2}+M_{3}+\delta_{0},
$$


in which

$$
\begin{aligned}
& M_{0}=\int_{T_{1}}^{0} e^{\epsilon s-\frac{2 \sqrt{k}\|\sigma \sigma\| q \|}{\sqrt{\mu}} s} K_{0} d s+\frac{e^{\epsilon_{0} T_{1}} K_{0}}{\epsilon_{0}}, \\
& M_{1}=K_{1}\left(\frac{\Gamma\left(\frac{3}{2}\right)}{\mu^{2} \sqrt{\pi}}\right)^{\frac{1}{2}} \int_{T_{1}}^{0} e^{\epsilon s-\frac{2 \sqrt{k} \mid\|\sigma\| q \|}{\sqrt{\mu}} s} d s+\frac{e^{\epsilon_{0} T_{1}} K_{2}}{\epsilon_{0} \sqrt{\pi \mu}}, \\
& M_{2}=K_{2}\left(\frac{\Gamma\left(\frac{5}{2}\right)}{\mu^{2} \sqrt{\pi}}\right)^{\frac{1}{2}} \int_{T_{1}}^{0} e^{\epsilon s-\frac{2 \sqrt{k}\|\sigma\| \mid\|\|}{\sqrt{\mu}} s} d s+\frac{e^{\epsilon_{0} T_{1}} K_{3}}{2 \epsilon_{0} \mu}, \\
& M_{3}=\frac{\sigma^{2}\|q(x)\|^{2}}{2 \mu} .
\end{aligned}
$$

Obviously, $M<\infty$. In addition, merging with Equations (25) and (A21)-(A23), we have

$$
\mathbb{E}(\rho(\omega)) \leq M<\infty .
$$

Appendix B.5. Proof of Lemma 9

For $\forall t \geq 0$, let

$$
\left\{\mathbf{U}_{n}\right\}_{n=1}^{+\infty}=\left\{\phi(\tau,-t, \omega) \mathbf{U}_{n}^{(0)}\right\}_{n=1}^{+\infty}=\left\{\left[u^{n}, \underline{v}^{n}\right]^{T}\right\}_{n=1}^{+\infty}, \forall \tau \in[-t, 0],
$$

By Lemma 8 , we find $\left\{\mathbf{U}_{n}\right\}_{n=1}^{+\infty}$ has a weakly star subsequence in $L^{\infty}\left([-t, 0], E_{1}\right)$, still denoted by $\left\{\mathbf{U}_{n}\right\}_{n=1}^{+\infty}$.

Taking the inner product of Equation (16) by $\mathbf{U}_{n}-\mathbf{U}_{m}$ in $E_{1}$, which, merging with Equation 5 and $\underline{v}=u_{t}+\varepsilon u$, gives

$$
\frac{d}{d t}\left\|\mathbf{U}_{n}-\mathbf{U}_{m}\right\|_{E_{1}}^{2} \leq-\varepsilon\left\|\mathbf{U}_{n}-\mathbf{U}_{m}\right\|_{E_{1}}^{2}+2 \bar{K}\left(\mathbf{U}_{m}, \mathbf{U}_{n}\right)
$$

where

$$
\begin{aligned}
\bar{K}\left(\mathbf{U}_{m}, \mathbf{U}_{n}\right) & =\left(\left[\mathcal{F}\left(u^{n}\right)+F_{0}, u^{n}\right]-\left[\mathcal{F}\left(u^{m}\right)+F_{0}, u^{m}\right], u_{t}^{n}-u_{t}^{m}\right) \\
& +\left(\left[\mathcal{F}\left(u^{n}\right)+F_{0}, u^{n}\right]-\left[\mathcal{F}\left(u^{m}\right)+F_{0}, u^{m}\right], \varepsilon\left(u^{n}-u^{m}\right)\right) .
\end{aligned}
$$

Since $\phi(0,-t, \omega)=S\left(t, \theta_{-t} \omega\right), \forall t \in \mathbb{R}^{+}, \omega \in \Omega$, then

$$
\left\|\mathbf{U}_{n}-\mathbf{U}_{m}\right\|_{E_{1}}^{2}\left(t, \theta_{-t} \omega\right) \leq e^{-\varepsilon t}\left\|\mathbf{U}_{n}^{(0)}-\mathbf{U}_{m}^{(0)}\right\|_{E_{1}}^{2}+2 \int_{-t}^{0} e^{\varepsilon s} \bar{K}\left(\mathbf{U}_{m}, \mathbf{U}_{n}\right)(s) d s .
$$

Notice that $\mathbf{U}_{n}^{(0)}, \mathbf{U}_{m}^{(0)} \in B$, then $e^{-\varepsilon t}\left\|\mathbf{U}_{n}^{(0)}-\mathbf{U}_{m}^{(0)}\right\|_{E_{1}}^{2} \rightarrow 0,(t \rightarrow \infty)$. Let

$$
T_{B}(\bar{\varepsilon})=\max \left\{-\frac{1}{\varepsilon} \log \left(\frac{\bar{\varepsilon}}{2\|B\|_{E_{1}}^{2}}\right), T_{B}(\omega)\right\} .
$$

thus, for $\forall \bar{\varepsilon}>0$, there exists

$$
T=\max \left\{T_{B}(\bar{\varepsilon}), T_{0}\right\}
$$

such that

$$
\left\|\mathbf{U}_{n}-\mathbf{U}_{m}\right\|_{E_{1}}^{2}\left(T, \theta_{-T} \omega\right) \leq \bar{\varepsilon}+2 \int_{-T}^{0} e^{\varepsilon s} \bar{K}\left(\mathbf{U}_{m}, \mathbf{U}_{n}\right)(s) d s
$$


Set

$$
K_{\bar{\varepsilon}, B, T, \omega}^{A}\left(\mathbf{U}_{m}^{(0)}, \mathbf{U}_{n}^{(0)}\right)=2 \int_{-T}^{0} e^{\varepsilon s} \bar{K}\left(\mathbf{U}_{m}, \mathbf{U}_{n}\right)(s) d s,
$$

Since $e^{\varepsilon s} \in L^{\infty}([-T, 0])$, along with Equation (A26), and Lemmas 3 and 4 , we obtain

$$
\liminf _{n \rightarrow+\infty} \liminf _{m \rightarrow+\infty} K_{\bar{\varepsilon}, B, T, \omega}^{A}\left(\mathbf{U}_{m}^{(0)}, \mathbf{U}_{n}^{(0)}\right)=0,
$$

Appendix B.6. Proof of Lemma 10

Taking the inner product of Equation (21) by $\boldsymbol{\Phi}=\left[\Phi_{1}, \Phi_{2}\right]^{T}=[u, v]^{T}$ in $E_{1}$, we have

$$
\frac{d}{d t}\|\boldsymbol{\Phi}\|_{E_{1}}^{2}=2\left(\mathbf{A}_{1} \boldsymbol{\Phi}, \boldsymbol{\Phi}\right)_{E_{1}}+2\left(\mathbf{F}_{2}\left(\boldsymbol{\Phi}, \theta_{t} \omega\right)+\mathbf{F}_{3}\left(\boldsymbol{\Phi}, \theta_{t} \omega\right), \boldsymbol{\Phi}\right)_{E_{1}} .
$$

Invoking Lemma 5, we find

$$
2\left(\mathbf{A}_{1} \boldsymbol{\Phi}, \boldsymbol{\Phi}\right)_{E_{1}} \leq-\varepsilon\|\boldsymbol{\Phi}\|_{E_{1}}^{2}-k_{0}\left\|\Phi_{2}\right\| .
$$

Since

$$
\begin{aligned}
& 2\left(\mathbf{F}_{2}\left(\boldsymbol{\Phi}, \theta_{t} \omega\right)+\mathbf{F}_{3}\left(\boldsymbol{\Phi}, \theta_{t} \omega\right), \boldsymbol{\Phi}\right)_{E_{1}} \\
= & 2\left(\sigma z\left(\theta_{t} \omega\right) \Phi_{1}, \Phi_{1}\right)_{2}+2\left(-\sigma z\left(\theta_{t} \omega\right) \Phi_{2}, \Phi_{2}\right)+2\left(\sigma^{2} z^{2}\left(\theta_{t} \omega\right) \Phi_{1}, \Phi_{2}\right) \\
& +2\left(N\left(\Phi_{1}\right), \Phi_{2}\right)
\end{aligned}
$$

by Equations (61)-(65), it can be obtained that

$$
\begin{aligned}
& \left(\mathbf{F}_{2}\left(\boldsymbol{\Phi}, \theta_{t} \omega\right)+\mathbf{F}_{3}\left(\boldsymbol{\Phi}, \theta_{t} \omega\right), \boldsymbol{\Phi}\right)_{E_{1}} \\
= & 2\left|\sigma z\left(\theta_{t} \omega\right)\right|\left\|A^{\frac{1}{2}} \Phi_{1}\right\|^{2}+2\left|\sigma z\left(\theta_{t} \omega\right)\right|\left\|\Phi_{2}\right\|^{2}+\frac{\sigma^{2}\left|z\left(\theta_{t} \omega\right)\right|^{2}}{\sqrt{\Lambda_{1}}}\left(\left\|A^{\frac{1}{2}} \Phi_{1}\right\|^{2}+\left\|\Phi_{2}\right\|^{2}\right) \\
& -\frac{k}{2} \frac{d}{d t}\left\|\Delta \mathcal{F}\left(\Phi_{1}\right)\right\|^{2}-2 k \varepsilon\left\|\Delta \mathcal{F}\left(\Phi_{1}\right)\right\|^{2}+2 k \sigma z\left(\theta_{t} \omega\right)\left\|\Delta \mathcal{F}\left(\Phi_{1}\right)\right\|^{2} \\
& -k \frac{d}{d t} \mathrm{Y}\left(\mathcal{F}\left(\Phi_{1}\right), F_{0}\right)-2 k \varepsilon Y\left(\mathcal{F}\left(\Phi_{1}\right), F_{0}\right)+2 k \sigma z\left(\theta_{t} \omega\right) Y\left(\mathcal{F}\left(\Phi_{1}\right), F_{0}\right) .
\end{aligned}
$$

Substituting Equations (A28)-(A29) into Equation (A27), we obtain

$$
\begin{aligned}
& \frac{d}{d t}\left(\left\|\boldsymbol{\Phi}_{1}\right\|_{E_{1}}^{2}+\frac{k}{2}\left\|\Delta \mathcal{F}\left(\Phi_{1}\right)\right\|^{2}+k Y\left(\mathcal{F}\left(\Phi_{1}\right), F_{0}\right)\right) \\
\leq & -\varepsilon\|\boldsymbol{\Phi}\|_{E_{1}}^{2}-\varepsilon \frac{k}{2}\left\|\Delta \mathcal{F}\left(\Phi_{1}\right)\right\|^{2}-\frac{3 \varepsilon k}{2}\left\|\Delta \mathcal{F}\left(\Phi_{1}\right)\right\|^{2}-\varepsilon k Y\left(\mathcal{F}\left(\Phi_{1}\right), F_{0}\right)-\varepsilon k Y\left(\mathcal{F}\left(\Phi_{1}\right), F_{0}\right) \\
& \left(2\left|\sigma z\left(\theta_{t} \omega\right)\right|+\frac{\sigma^{2}\left|z\left(\theta_{t} \omega\right)\right|^{2}}{\sqrt{\Lambda_{1}}}\right)\left(\left\|A^{\frac{1}{2}} \Phi_{1}\right\|^{2}+\left\|\Phi_{2}\right\|^{2}+k\left\|\Delta \mathcal{F}\left(\Phi_{1}\right)\right\|^{2}+3 k Y\left(\mathcal{F}\left(\Phi_{1}\right), F_{0}\right)\right) \\
& +4\left(2\left|\sigma z\left(\theta_{t} \omega\right)\right|+\frac{\sigma^{2}\left|z\left(\theta_{t} \omega\right)\right|^{2}}{\sqrt{\Lambda_{1}}}\right) k\left|\mathrm{Y}\left(\mathcal{F}\left(\Phi_{1}\right), F_{0}\right)\right| .
\end{aligned}
$$


along with Equations (66) and (67), we have

$$
\begin{aligned}
& \frac{d}{d t}\left(\left\|\Phi_{1}\right\|_{E_{1}}^{2}+\frac{k}{2}\left\|\Delta \mathcal{F}\left(\Phi_{1}\right)\right\|^{2}+k Y\left(\mathcal{F}\left(\Phi_{1}\right), F_{0}\right)\right) \\
\leq & -\varepsilon\left(\left\|\Phi_{1}\right\|_{E_{1}}^{2}+\frac{k}{2}\left\|\Delta \mathcal{F}\left(\Phi_{1}\right)\right\|^{2}+k Y\left(\mathcal{F}\left(\Phi_{1}\right), F_{0}\right)\right) \\
& +3\left(2\left|\sigma z\left(\theta_{t} \omega\right)\right|+\frac{\sigma^{2}\left|z\left(\theta_{t} \omega\right)\right|^{2}}{\sqrt{\Lambda_{1}}}\right)\left(\left\|A^{\frac{1}{2}} \Phi_{1}\right\|^{2}+\left\|\Phi_{2}\right\|^{2}+\frac{k}{2}\left\|\Delta \mathcal{F}\left(\Phi_{1}\right)\right\|^{2}+k Y\left(\mathcal{F}\left(\Phi_{1}\right), F_{0}\right)\right) \\
& +\varepsilon k C\left(\frac{3}{2}, C_{1}, C_{2}\right)\left\|F_{0}\right\|_{2}^{2}+4 k\left(2\left|\sigma z\left(\theta_{t} \omega\right)\right|+\frac{\sigma^{2}\left|z\left(\theta_{t} \omega\right)\right|^{2}}{\sqrt{\Lambda_{1}}}\right) C\left(\frac{1}{8}, C_{1}, C_{2}\right)\left\|F_{0}\right\|_{2}^{2}
\end{aligned}
$$

Let

$$
\begin{gathered}
\mathcal{E}_{1}(t, \tau, \omega)=\overline{\mathcal{E}}_{1}\left(\Phi_{1}, \Phi_{2}, \omega\right)=\left(\left\|\Phi_{2}\right\|^{2}+\left\|A^{\frac{1}{2}} \Phi_{1}\right\|^{2}+\frac{k}{2}\left\|\Delta \mathcal{F}\left(\Phi_{1}\right)\right\|^{2}+k Y\left(\mathcal{F}, F_{0}\right)\right) \\
\mathcal{E}(t, \tau, \omega)=\overline{\mathcal{E}}\left(\Phi_{1}, \Phi_{2}, \omega\right)=\left(\left\|\Phi_{2}\right\|^{2}+\left\|A^{\frac{1}{2}} \Phi_{1}\right\|^{2}+\frac{k}{2}\left\|\Delta \mathcal{F}\left(\Phi_{1}\right)\right\|^{2}\right)
\end{gathered}
$$

applying Equation (53), we attain

$$
\overline{\mathcal{E}}\left(\Phi_{1}, \Phi_{2}, \omega\right) \leq \overline{\mathcal{E}}_{1}\left(\Phi_{1}, \Phi_{2}, \omega\right)+k C\left(\frac{1}{2}, C_{1}, C_{2}\right)\left\|F_{0}\right\|_{2}^{2}
$$

according to Equation (A30), we have

$$
\frac{d}{d t} \overline{\mathcal{E}}_{1}\left(\Phi_{1}, \Phi_{2}, \omega\right) \leq-\varepsilon \overline{\mathcal{E}}_{1}\left(\Phi_{1}, \Phi_{2}, \omega\right)+C_{0}\left(\theta_{t} \omega\right) \overline{\mathcal{E}}_{1}\left(\Phi_{1}, \Phi_{2}, \omega\right)+K
$$

where $C_{0}\left(\theta_{t} \omega\right)$ is given by Equation (29) and $K$ is denoted by Equation (31).

It follows form Equation (A32) that

$$
\overline{\mathcal{E}}_{1}\left(\Phi_{1}, \Phi_{2}, \omega\right) \leq e^{-\varepsilon(t-\tau)+\int_{\tau}^{t} C_{0}\left(\theta_{s} \omega\right) d s} \overline{\mathcal{E}}_{1}\left(\Phi_{1}, \Phi_{2}, \omega\right)+\int_{\tau}^{t} e^{-\varepsilon(t-s)+\int_{s}^{t} C_{0}\left(\theta_{k} \omega\right) d k} K d s,
$$

which means that for any given $t \geq \tau, t, \tau \in \mathbb{R}, \omega \in \Omega$, the following holds

$$
\mathcal{E}_{1}(t, \tau, \omega) \leq e^{-\varepsilon(t-\tau)+\int_{\tau}^{t} C_{0}\left(\theta_{s} \omega\right) d s} \mathcal{E}_{1}(\tau, \tau, \omega)+\int_{\tau}^{t} e^{-\varepsilon(t-s)+\int_{s}^{t} C_{0}\left(\theta_{k} \omega\right) d k} K d s .
$$

Since

$$
\lim _{t \rightarrow-\infty}-\frac{1}{t} \int_{0}^{t} C_{0}\left(\theta_{s} \omega\right) d s=-\frac{3 \sigma^{2}}{2 \mu \sqrt{\lambda_{1}}}-\frac{6 \sigma}{\sqrt{\pi \mu}}
$$

then, there exits $T_{1}<0$ such that for any $t<T_{1}$, the ensuing can be satisfied

$$
\lim _{t \rightarrow-\infty}-\frac{1}{t} \int_{0}^{t} C_{0}\left(\theta_{s} \omega\right) d s \geq-\frac{3 \sigma^{2}}{\mu \sqrt{\lambda_{1}}}-\frac{12 \sigma}{\sqrt{\pi \mu}}
$$

which together with Equation (28) gives that $\epsilon=\varepsilon-\frac{3 \sigma^{2}}{\mu \sqrt{\lambda_{1}}}-\frac{12 \sigma}{\sqrt{\pi \mu}}>0$; hence, when $t<T_{1}$,

$$
\varepsilon t+\int_{t}^{0} C_{0}\left(\theta_{s} \omega\right) d s \leq \epsilon t<0,
$$


therefore

$$
\begin{aligned}
& e^{\varepsilon t+\int_{t}^{0} C_{0}\left(\theta_{s} \omega\right) d s} \mathcal{E}(t, t, \omega) \rightarrow 0, t \rightarrow-\infty . \\
& \int_{-\infty}^{0} e^{\varepsilon t+\int_{t}^{0} C_{0}\left(\theta_{s} \omega\right) d s} K_{0} d t \\
= & \int_{T_{1}}^{0} e^{\varepsilon t+\int_{t}^{0} C_{0}\left(\theta_{s} \omega\right) d s} K_{0} d s+\frac{e^{\epsilon T_{1}} K_{0}}{\epsilon}, \\
& \int_{-\infty}^{0} e^{\varepsilon t+\int_{t}^{0} C_{0}\left(\theta_{s} \omega\right) d s}\left|z\left(\theta_{t} \omega\right)\right| K_{1} d t \\
= & \int_{T_{1}}^{0} e^{\varepsilon t+\int_{t}^{0} C_{0}\left(\theta_{s} \omega\right) d s}\left|z\left(\theta_{t} \omega\right)\right| K_{1} d s+\int_{-\infty}^{T_{1}} e^{\varepsilon s}\left|z\left(\theta_{s} \omega\right)\right| K_{1} d s . \\
& \int_{-\infty}^{t} e^{\varepsilon t+\int_{t}^{0} C_{0}\left(\theta_{s} \omega\right) d s}\left|z\left(\theta_{s} \omega\right)\right|^{2} K_{2} d t \\
= & \int_{T_{1}}^{t} e^{\varepsilon t+\int_{t}^{0} C_{0}\left(\theta_{s} \omega\right) d s}\left|z\left(\theta_{t} \omega\right)\right|^{2} K_{2} d s+\int_{-\infty}^{T_{1}} e^{\epsilon s}\left|z\left(\theta_{s} \omega\right)\right|^{2} K_{2} d s .
\end{aligned}
$$

Combining Equation (31) with the fact that $z\left(\theta_{t} \omega\right)$ is tempered, we have that $\rho_{0}(\omega)$ defined by Equation (29) is a bounded random variable ( $\mathbb{P}$-a.s.). Furthermore, merging with Equation (A31), we find the random variable $\rho_{0}(\omega)$ in Equation (30) is also bounded.

Incorporating Equation (A40), it can be derived that. for any initial value $\boldsymbol{\Phi} \in B$, there exists $\bar{T}_{B}(\omega)<0$ such that

$$
\mathcal{E}(0, t, \omega) \leq \sqrt{\rho(\omega)}, \quad \forall t \leq \bar{T}_{B}(\omega)
$$

Based on the relationship between $v$ and $u$, we have that. for any initial value $[u, v]^{T} \in E_{1}$, the following holds

$$
\left(\left\|A^{\frac{1}{2}} u\right\|^{2}+\left\|u_{t}+\varepsilon u\right\|^{2}\right)_{E_{1}}(0, t, \omega) \leq\left(\frac{|\sigma z(\omega)|^{2}}{\Lambda_{1}}+1\right) \rho(\omega), \forall t \leq \bar{T}_{B}(\omega) .
$$

Let $t=-\bar{t}, T_{B}(\omega)=-\bar{T}_{B}(\omega)$; along with Equation (32), it can be asserted that, for any given non-random bounded set $B \subset E_{1}$, there exists $T_{B}(\omega)>0$ such that for $\forall t>T_{B}(\omega)$, the following holds $\mathbb{P}$-a.s.

$$
\left\|S\left(t, \theta_{-t} \omega\right) B\right\|_{E_{1}} \leq r(\omega) .
$$

Thus, $S(t, \omega)$ possesses global random absorbing set in $E_{1}$.

The remainder of this proof is intended to estimate the expectation of $\rho(\omega)$. It follows from Equations (35), (36) and (29) that

$$
\mathbb{E}\left(e^{\epsilon S+\int_{S}^{0} C_{0}\left(\theta_{k} \omega\right) d k}\right) \leq e^{\epsilon \mathcal{S}-\frac{6|\sigma|}{\sqrt{\mu}} S-\frac{3 \sigma^{2}}{\mu \sqrt{\Lambda_{1}}} S}
$$

Merging with Equation (A21) and Cauchy inequality, we have

$$
\begin{aligned}
& \mathbb{E}\left(e^{\varepsilon S+\int_{s}^{0} C_{0}\left(\theta_{k} \omega\right) d k}\left|z\left(\theta_{S} \omega\right)\right|^{2}\right) \leq e^{\epsilon S-\frac{6|\sigma|}{\sqrt{\mu}} s-\frac{3 \sigma^{2}}{\mu \sqrt{\Lambda_{1}}} s}\left(\frac{\Gamma\left(\frac{5}{2}\right)}{\mu^{2} \sqrt{\pi}}\right)^{\frac{1}{2}}, \\
& \mathbb{E}\left(e^{\varepsilon S+\int_{s}^{0} C_{0}\left(\theta_{k} \omega\right) d k}\left|z\left(\theta_{s} \omega\right)\right|\right) \leq e^{\epsilon S-\frac{6|\sigma|}{\sqrt{\mu}} s-\frac{3 \sigma^{2}}{\mu \sqrt{\Lambda_{1}}} s}\left(\frac{\Gamma\left(\frac{3}{2}\right)}{\mu^{2} \sqrt{\pi}}\right)^{\frac{1}{2}} .
\end{aligned}
$$


Let

$$
M=M_{0}+M_{1}+M_{2}+k C\left(\frac{1}{2}, C_{1}, C_{2}\right)\left\|F_{0}\right\|_{2}^{2}+\delta_{0},
$$

in which

$$
\begin{aligned}
& M_{0}=\int_{T_{1}}^{0} e^{\epsilon s-\frac{6|\sigma| \sigma \mid}{\sqrt{\mu}} s-\frac{3 \sigma^{2}}{\mu \sqrt{\Lambda_{1}}} s} K_{0} d s+\frac{e^{\epsilon} T_{1}}{\epsilon} K_{0} \\
& M_{1}=K_{1}\left(\frac{\Gamma\left(\frac{3}{2}\right)}{\mu^{2} \sqrt{\pi}}\right)^{\frac{1}{2}} \int_{T_{1}}^{0} e^{\epsilon s-\frac{6|\sigma|}{\sqrt{\mu}} s-\frac{3 \sigma^{2}}{\mu \sqrt{\Lambda_{1}}} s} d s+\frac{e^{\epsilon} T_{1} K_{2}}{\epsilon \sqrt{\pi \mu}}, \\
& M_{2}=K_{2}\left(\frac{\Gamma\left(\frac{5}{2}\right)}{\mu^{2} \sqrt{\pi}}\right)^{\frac{1}{2}} \int_{T_{1}}^{0} e^{\epsilon s-\frac{6|\sigma|}{\sqrt{\mu}} s-\frac{3 \sigma^{2}}{\mu \sqrt{\Lambda_{1}}} s} d s+\frac{e^{\epsilon} T_{1} K_{3}}{2 \epsilon \mu} .
\end{aligned}
$$

Together with Equations (30), (A41), (A42) and (A37)-(A39), we have

$$
\mathbb{E}(\rho(\omega)) \leq M<\infty .
$$

Appendix B.7. Proof of Lemma 11

For $\forall t \geq 0, \forall \tau \in[-t, 0]$, let $\left\{\boldsymbol{\Phi}_{n}^{(0)}\right\}_{n=1}^{+\infty}=\left\{T\left(\theta_{-t} \omega\right) \mathbf{U}_{n}^{(0)}\right\}_{n=1}^{+\infty}$,

$$
\begin{aligned}
& \left\{\mathbf{U}_{n}\right\}_{n=1}^{+\infty}=\left\{\phi(\tau,-t, \omega) \mathbf{U}_{n}^{(0)}\right\}_{n=1}^{+\infty}=\left\{\left[u^{n}, \underline{v}^{n}\right]^{T}\right\}_{n=1}^{+\infty}, \\
& \left\{\boldsymbol{\Phi}_{n}\right\}_{n=1}^{+\infty}=\left\{\varphi(\tau,-t, \omega) \boldsymbol{\Phi}_{n}^{(0)}\right\}_{n=1}^{+\infty}=\left\{\left[u^{n}, v^{n}\right]^{T}\right\}_{n=1}^{+\infty} .
\end{aligned}
$$

By Lemma 10, we find that $\left\{\mathbf{U}_{n}\right\}_{n=1}^{+\infty},\left\{\boldsymbol{\Phi}_{n}\right\}_{n=1}^{+\infty}$ have weakly star convergence in $L^{\infty}\left([-t, 0], E_{1}\right)$, still denoted by $\left\{\mathbf{U}_{n}\right\}_{n=1}^{+\infty},\left\{\boldsymbol{\Phi}_{n}\right\}_{n=1}^{+\infty}$. Obviously $\boldsymbol{\Phi}_{n}-\boldsymbol{\Phi}_{m}$ satisfies Equation (22). Taking the inner product of Equation (22) by $\boldsymbol{\Phi}_{n}-\boldsymbol{\Phi}_{m}$ in $E_{1}$, we have

$$
\begin{aligned}
\frac{d}{d t}\left\|\boldsymbol{\Phi}_{n}-\boldsymbol{\Phi}_{m}\right\|_{E_{1}}^{2}= & 2\left(\mathbf{A}_{1}\left(\boldsymbol{\Phi}_{n}-\boldsymbol{\Phi}_{m}\right), \boldsymbol{\Phi}_{n}-\boldsymbol{\Phi}_{m}\right)_{E_{1}} \\
& +2\left(\mathbf{F}_{2}\left(\boldsymbol{\Phi}_{n}, \theta_{t} \omega\right)-\mathbf{F}_{2}\left(\boldsymbol{\Phi}_{m}, \theta_{t} \omega\right), \boldsymbol{\Phi}_{n}-\boldsymbol{\Phi}_{m}\right)_{E_{1}} \\
& +2\left(\mathbf{F}_{3}\left(\boldsymbol{\Phi}_{n}, \theta_{t} \omega\right)-\mathbf{F}_{3}\left(\boldsymbol{\Phi}_{m}, \theta_{t} \omega\right), \boldsymbol{\Phi}_{n}-\boldsymbol{\Phi}_{m}\right)_{E_{1}}
\end{aligned}
$$

In light of Lemma 5, we have

$$
2\left(\mathbf{A}_{1}\left(\boldsymbol{\Phi}_{n}-\boldsymbol{\Phi}_{m}\right), \boldsymbol{\Phi}_{n}-\boldsymbol{\Phi}_{m}\right)_{E_{1}} \leq-\varepsilon\left\|\boldsymbol{\Phi}_{n}-\boldsymbol{\Phi}_{m}\right\|_{E_{1}}^{2}-k_{0}\left\|\Phi_{2, n}-\Phi_{2, m}\right\| .
$$

In term of Equations (61)-(63), we find

$$
\begin{aligned}
& 2\left(\mathbf{F}_{2}\left(\boldsymbol{\Phi}_{n}, \theta_{t} \omega\right)-\mathbf{F}_{2}\left(\boldsymbol{\Phi}_{m}, \theta_{t} \omega\right)+\mathbf{F}_{3}\left(\boldsymbol{\Phi}_{n}, \theta_{t} \omega\right)-\mathbf{F}_{3}\left(\boldsymbol{\Phi}_{m}, \theta_{t} \omega\right), \boldsymbol{\Phi}\right)_{E_{1}} \\
\leq & 2\left|\sigma z\left(\theta_{t} \omega\right)\right|\left\|A^{\frac{1}{2}} \Phi_{1}\right\|^{2}+2\left|\sigma z\left(\theta_{t} \omega\right)\right|\left\|\Phi_{2}\right\|^{2}+\frac{\sigma^{2}\left|z\left(\theta_{t} \omega\right)\right|^{2}}{\sqrt{\Lambda_{1}}}\left(\left\|A^{\frac{1}{2}} \Phi_{1}\right\|^{2}+\left\|\Phi_{2}\right\|^{2}\right) \\
& +\left(\left(N\left(u_{n}\right)-N\left(u_{m}\right), v_{n}-v_{m}\right),\right.
\end{aligned}
$$

Merging with Equation (29), we obtain

$$
\begin{aligned}
& 2\left(\mathbf{F}_{2}\left(\boldsymbol{\Phi}_{n}, \theta_{t} \omega\right)-\mathbf{F}_{2}\left(\boldsymbol{\Phi}_{m}, \theta_{t} \omega\right)+\mathbf{F}_{3}\left(\boldsymbol{\Phi}_{n}, \theta_{t} \omega\right)-\mathbf{F}_{3}\left(\boldsymbol{\Phi}_{m}, \theta_{t} \omega\right), \boldsymbol{\Phi}\right)_{E_{1}} \\
\leq & \frac{1}{3} C_{0}\left(\theta_{t} \omega\right)\left\|\mathbf{\Phi}_{n}-\boldsymbol{\Phi}_{m}\right\|_{E_{1}}^{2}+\left(\left(N\left(u^{n}\right)-N\left(u^{m}\right), v^{n}-v^{m}\right) .\right.
\end{aligned}
$$


Since $v=u_{t}+\varepsilon u_{t}-\sigma u z\left(\theta_{t} \omega\right)$, then

$$
\begin{aligned}
& 2\left(\mathbf{F}_{2}\left(\boldsymbol{\Phi}_{n}, \theta_{t} \omega\right)-\mathbf{F}_{2}\left(\boldsymbol{\Phi}_{m}, \theta_{t} \omega\right)+\mathbf{F}_{3}\left(\boldsymbol{\Phi}_{n}, \theta_{t} \omega\right)-\mathbf{F}_{3}\left(\boldsymbol{\Phi}_{m}, \theta_{t} \omega\right), \boldsymbol{\Phi}\right)_{E_{1}} \\
\leq & \frac{1}{3} C_{0}\left(\theta_{t} \omega\right)\left\|\boldsymbol{\Phi}_{n}-\boldsymbol{\Phi}_{m}\right\|_{E_{1}}^{2}+\bar{K}\left(\mathbf{U}_{m}, \mathbf{U}_{n}\right)+\sigma z\left(\theta_{t} \omega\right) \widehat{K}\left(\mathbf{U}_{m}, \mathbf{U}_{n}\right) .
\end{aligned}
$$

where $\bar{K}\left(\mathbf{U}_{m}, \mathbf{U}_{n}\right)$ is denoted by Equation (A26)

$$
\widehat{K}\left(\mathbf{U}_{m}, \mathbf{U}_{n}\right)=\left(\left[\mathcal{F}\left(u^{n}\right)+F_{0}, u^{n}\right]-\left[\mathcal{F}\left(u^{m}\right)+F_{0}, u^{m}\right], u^{n}-u^{m}\right) .
$$

Substituting Equations (A45) and (A46) into Equation (A44), it can be obtained

$$
\begin{aligned}
\frac{d}{d t}\left\|\boldsymbol{\Phi}_{n}-\boldsymbol{\Phi}_{m}\right\|_{E_{1}}^{2} \leq & -\varepsilon\left\|\boldsymbol{\Phi}_{n}-\boldsymbol{\Phi}_{m}\right\|_{E_{1}}^{2}+\frac{2}{3} C_{0}\left(\theta_{t} \omega\right)\left\|\boldsymbol{\Phi}_{n}-\boldsymbol{\Phi}_{m}\right\|_{E_{1}}^{2} \\
& +2 \bar{K}\left(\mathbf{U}_{m}, \mathbf{U}_{n}\right)+2 \sigma z\left(\theta_{t} \omega\right) \widehat{K}\left(\mathbf{U}_{m}, \mathbf{U}_{n}\right),
\end{aligned}
$$

hence

$$
\begin{aligned}
& \left\|\boldsymbol{\Phi}_{n}-\boldsymbol{\Phi}_{m}\right\|_{E_{1}}^{2}(0,-t, \omega) \\
\leq & e^{-\varepsilon t+\int_{-t}^{0} \frac{2}{3} C_{0}\left(\theta_{k} \omega\right) d k}\left\|\boldsymbol{\Phi}_{n}-\boldsymbol{\Phi}_{m}\right\|_{E_{1}}^{2}(-t,-t, \omega) \\
& +2 \int_{-t}^{0} e^{-\varepsilon s+\int_{-s}^{0} \frac{2}{3} C_{0}\left(\theta_{k} \omega\right) d k}\left(\bar{K}\left(\mathbf{U}_{m}, \mathbf{U}_{n}\right)+\sigma z\left(\theta_{t} \omega\right) \widehat{K}\left(\mathbf{U}_{m}, \mathbf{U}_{n}\right)\right) d s .
\end{aligned}
$$

By Equation (A35), we have that for any given $T_{0} \geq-\bar{T}, \forall t>T_{0}$, the following holds

$$
\begin{aligned}
& \left\|\boldsymbol{\Phi}_{n}-\boldsymbol{\Phi}_{m}\right\|_{E_{1}}^{2}(0,-t, \omega) \\
\leq & e^{-\epsilon t}\left\|\boldsymbol{\Phi}_{n}-\boldsymbol{\Phi}_{m}\right\|_{E_{1}}^{2}(-t,-t, \omega) \\
& +2 \int_{-t}^{0} e^{-\varepsilon s+\int_{-s}^{0} \frac{2}{3} C_{0}\left(\theta_{k} \omega\right) d k}\left(\bar{K}\left(\mathbf{U}_{m}, \mathbf{U}_{n}\right)+\sigma z\left(\theta_{s} \omega\right) \widehat{K}\left(\mathbf{U}_{m}, \mathbf{U}_{n}\right)\right) d s,
\end{aligned}
$$

and

$$
e^{-\epsilon t}\left\|\boldsymbol{\Phi}_{n}-\boldsymbol{\Phi}_{m}\right\|_{E_{1}}^{2}(-t,-t, \omega) \rightarrow 0, t \rightarrow+\infty .
$$

Hence, for any $\forall \bar{\varepsilon}>0$, let

$$
T_{B}(\bar{\varepsilon})=\max \left\{-\frac{1}{\epsilon} \log \left(\frac{\bar{\varepsilon}}{4\left(\frac{|\sigma z(\omega)|^{2}}{\Lambda_{1}}+1\right)\|B\|_{E_{1}}^{2}}\right), T_{B}(\omega)\right\} .
$$

then, it follows from Equations (A50) and (A51) that there exists

$$
T=\max \left\{T_{B}(\bar{\varepsilon}), T_{0}\right\},
$$

such that

$$
\left\|\boldsymbol{\Phi}_{n}-\boldsymbol{\Phi}_{m}\right\|_{E_{1}}^{2}\left(T, \theta_{-T} \omega\right) \leq \frac{\bar{\varepsilon}}{\left(\frac{|\sigma z(\omega)|^{2}}{\Lambda_{1}}+1\right)}+2 \int_{-T}^{0} e^{-\varepsilon s+\int_{-s}^{0} \frac{2}{3} C_{0}\left(\theta_{k} \omega\right) d k} \mathbb{K}\left(s, \boldsymbol{\Phi}_{m}, \boldsymbol{\Phi}_{n}\right) d s .
$$

where $T_{B}(\omega)$ is defined in Lemma 10 ,

$$
\mathbb{K}\left(T, \boldsymbol{\Phi}_{m}, \boldsymbol{\Phi}_{n}\right)=\int_{-T}^{0} e^{-\varepsilon s+\int_{-s}^{0} \frac{2}{3} C_{0}\left(\theta_{k} \omega\right) d k}\left(\bar{K}\left(\mathbf{U}_{m}, \mathbf{U}_{n}\right)+\sigma z\left(\theta_{s} \omega\right) \widehat{K}\left(\mathbf{U}_{m}, \mathbf{U}_{n}\right)\right) d s,
$$


employ the relationship between $\left\{\mathbf{U}_{n}\right\}_{n=1}^{+\infty}$ and $\left\{\boldsymbol{\Phi}_{n}\right\}_{n=1}^{+\infty}$, we derive

$$
\begin{aligned}
\left\|\mathbf{U}_{n}-\mathbf{U}_{m}\right\|_{E_{1}}^{2}\left(T, \theta_{-T} \omega\right) & \leq\left(\frac{|\sigma z(\omega)|^{2}}{\Lambda_{1}}+1\right)\left(\frac{\bar{\varepsilon}}{\left(\frac{|\sigma z(\omega)|^{2}}{\Lambda_{1}}+1\right)}+\mathbb{K}\left(T, \boldsymbol{\Phi}_{m}, \boldsymbol{\Phi}_{n}\right)\right) \\
& =\bar{\varepsilon}+2\left(\frac{|\sigma z(\omega)|^{2}}{\Lambda_{1}}+1\right) \mathbb{K}\left(T, \boldsymbol{\Phi}_{m}, \boldsymbol{\Phi}_{n}\right) .
\end{aligned}
$$

Let

$$
K_{\bar{\varepsilon}, B, T, \omega}^{M}\left(\mathbf{U}_{m}^{(0)}, \mathbf{U}_{n}^{(0)}\right)=2\left(\frac{|\sigma z(\omega)|^{2}}{\Lambda_{1}}+1\right) \mathbb{K}\left(T, \boldsymbol{\Phi}_{m}, \boldsymbol{\Phi}_{n}\right)
$$

then

$$
\left\|\mathbf{U}_{n}-\mathbf{U}_{m}\right\|_{E_{1}}^{2}\left(T, \theta_{-T} \omega\right) \leq \bar{\varepsilon}+K_{\bar{\varepsilon}, B, T, \omega}^{M}\left(\mathbf{U}_{m}^{(0)}, \mathbf{U}_{n}^{(0)}\right) .
$$

along with Equation (1), we have $e^{-\varepsilon s+\int_{-s}^{0} \frac{2}{3} C_{0}\left(\theta_{k} \omega\right) d k} \in L^{\infty}([-T, 0]), z\left(\theta_{t} \omega\right) \in \mathbf{C}([-T, 0])$, which together with Lemmas 3 and 4 gives

$$
\liminf _{n \rightarrow+\infty} \liminf _{m \rightarrow+\infty} K_{\bar{\varepsilon}, B, T, \omega}^{M}\left(\mathbf{U}_{m}^{(0)}, \mathbf{U}_{n}^{(0)}\right)=0,
$$

\section{References}

1. Amabili, M. Nonlinear Vibrations and Stability of Shells and Plates; Cambridge University Press: Cambridge, UK, 2008.

2. Lagnese, J.E. Boundary Stabilization of Thin Plates; SIAM: Philadelphia, PA, USA, 1989.

3. Ciarlet, P.G. Mathematical Elasticity: Theory of Plates; Elsevier: Amsterdam, The Netherlands, 1997.

4. Arnold, L. Random Dynamical Systems; Springer: Berlin, Germany, 1998.

5. Crauel, H.; Flandoli, F. Attractors for random dynamical systems. Probab. Theory Relat. Fields 1994, 100, 365-393. [CrossRef]

6. Schmalfuss, B. Measure Attractors and Stochastic Attractors, Institut for Dynamische Systeme; Technical Report; Bermen University: Bermen, Germany, 1995.

7. Crauel, H.; Debussche, A.; Flandoli, F. Random attractors. J. Dyn. Differ. Equ. 1997, 9, 307-341. [CrossRef]

8. Crauel, $\mathrm{H}$. Global random attractors are uniquely determined by attracting deterministic compact sets. Ann. Mat. Pura Appl. 1999, 176, 57-72. [CrossRef]

9. Bates, P.W.; Lu, K.; Wang, B. Random attractors for stochastic reaction-diffusion equations on unbounded domains. J. Differ. Equ. 2009, 246, 845-869. [CrossRef]

10. Caraballo, T.; Langa, J.A.; Robinson, J.C. Stability and random attractors for a reaction-diffusion equation with multiplicative noise. Discret. Contin. Dyn. Syst. 2000, 6, 875-892.

11. Zhou, S.; Yin, F.; Ouyang, Z. Random attractor for damped nonlinear wave equations with white noise. SIAM J. Appl. Dyn. Syst. 2005, 4, 883-903. [CrossRef]

12. Fan, X.; Chen, H. Attractors for the stochastic reaction-diffusion equation driven by linear multiplicative noise with a variable coefficient. J. Math. Anal. Appl. 2013, 398, 715-728. [CrossRef]

13. Fan, $X$. Attractors for a damped stochastic wave equation of Sine-Gordon type with sublinear multiplicative noise. Stochastic Anal. Appl. 2006, 24, 767-793. [CrossRef]

14. You, Y. Global Attractor for Nonlinear Wave Equations with Critical Exponent on Unbounded Domain. Appl. Math. Nonlinear Sci. 2016, 2, 581-602. [CrossRef]

15. Pappalardo, C.M.; Guida, D. Use of the Adjoint Method for Controlling the Mechanical Vibrations of Nonlinear Systems. Machines 2018, 6, 19. [CrossRef]

16. Fortuna, L.; Muscato, G. A roll stabilization system for a monohull ship: Modeling, identification, and adaptive control. IEEE Trans. Control Syst. Technol. 1996, 4, 18-28. [CrossRef] 
17. Pappalardo, C.M.; Guida, D. System Identification Algorithm for Computing the Modal Parameters of Linear Mechanical Systems. Machines 2018, 6, 12. [CrossRef]

18. Pappalardo, C.M.; Guida, D. On the Computational Methods for Solving the Differential-Algebraic Equations of Motion of Multibody Systems. Machines 2018, 6, 20. [CrossRef]

19. Pappalardo, C.M.; Zhang, Z.; Shabana, A.A. Use of independent volume parameters in the development of new large displacement ANCF triangular plate/shell elements. Nonlinear Dyn. 2018, 91, 2171-2202. [CrossRef]

20. Pappalardo, C.M.; Wallin, M.; Shabana, A.A. A new ANCF/CRBF fully parameterized plate finite element. J. Comput. Nonlinear Dyn. 2017, 12, 031008. [CrossRef]

21. Pappalardo, C.M.; Yu, Z.; Zhang, X.; Shabana, A.A. Rational ANCF thin plate finite element. J. Comput. Nonlinear Dyn. 2016, 11, 051009. [CrossRef]

22. Lasiecka, I. Weak, classical and intermediate solutions to full von Kármán system of dynamic nonlinear elasticity. Appl. Anal. 1998, 68, 121-145.

23. Leiva, H.; Sivoli, Z. Existence, stability and smoothness of a bounded solution for nonlinear time-varying thermoelastic plate equations. J. Math. Anal. Appl. 2003, 285, 191-211. [CrossRef]

24. Abels, H.; Mora, M.G.; Müller, S. The time-dependent von Kármán plate equation as a limit of $3 \mathrm{~d}$ nonlinear elasticity. Calc. Var. Partial Differ. Equ. 2011, 41, 241-259. [CrossRef]

25. Favini, A.; Horn, M.A.; Lasiecka, I.; Tataru, D. Global existence, uniqueness and regularity of solutions to a von Karman system with nonlinear boundary dissipation. Differ. Integral Equ. 1996, 9, 267-294.

26. Chueshov, I.; Lasiecka, I. Von Karman Evolution Equations: Well-Posedness and Long Time Dynamics; Springer Science \& Business Media: Berlin/Heidelberg, Germany, 2010.

27. Chueshov, I.; Lasiecka, I. Attractors for second-order evolution equations with a nonlinear damping. J. Dyn. Differ. Equ. 2004, 16, 469-512. [CrossRef]

28. Chueshov, I.; Lasiecka, I. Inertial manifolds for von Kármán plate equations. Appl. Math. Optim. 2002, $46,179-206$.

29. Lasiecka, I. Uniform decay rates for full von karman system of dynamic theromelasticity with free boundary conditions and partial boundary dissipation. Commun. Partial Differ. Equ. 1999, 24, 1801-1847. [CrossRef]

30. Ryzhkova, I. Dynamics of a thermoelastic von Karman plate in a subsonic gas flow. Zeitschrift für angewandte Mathematik und Physik 2007, 58, 246-261. [CrossRef]

31. Yeoul Park, J.; Hye Park, S. Uniform decay for a von Karman plate equation with a boundary memory condition. Math. Methods Appl. Sci. 2005, 28, 2225-2240. [CrossRef]

32. Park, S.H. Long-time dynamics of a von Karman equation with time delay. Appl. Math. Lett. 2018, 75, 128-134. [CrossRef]

33. Chueshov, I.; Lasiecka, I.; Webster, J.T. Attractors for delayed, nonrotational von Karman plates with applications to flow-structure interactions without any damping. Commun. Partial Differ. Equ. 2014, 39, 1965-1997. [CrossRef]

34. Lasiecka, I.; Webster, J.T. Eliminating flutter for clamped von Karman plates immersed in subsonic flows. Commun. Pure Appl. Anal. 2014, 13, 1935-1969 . [CrossRef]

35. Khanmamedov, A.K. Global attractors for von Karman equations with nonlinear interior dissipation. J. Math. Anal. Appl. 2006, 318, 92-101. [CrossRef]

36. Chen, H.; Cao, D.; Jiang, J. Random Attractors for Von Karman Plates Subjected to Multiplicative White Noise Loadings. In Dynamical Systems: Theoretical and Experimental Analysis; Springer: New York, NY, USA, 2016; pp. 59-70.

37. Awrejcewicz, J.; Krysko, A. Analysis of complex parametric vibrations of plates and shells using Bubnov-Galerkin approach. Arch. Appl. Mech. 2003, 73, 495-504. [CrossRef]

38. Alijani, F.; Bakhtiari-Nejad, F.; Amabili, M. Nonlinear vibrations of FGM rectangular plates in thermal environments. Nonlinear Dyn. 2011, 66, 251. [CrossRef]

39. Park, J.S.; Kim, J.H. Thermal postbuckling and vibration analyses of functionally graded plates. J. Sound Vib. 2006, 289, 77-93. [CrossRef]

40. Rashidi, M.; Shooshtari, A.; Bég, O.A. Homotopy perturbation study of nonlinear vibration of Von Karman rectangular plates. Comput. Struct. 2012, 106, 46-55. [CrossRef]

41. Ghayesh, M.H.; Amabili, M.; Païdoussis, M.P. Nonlinear dynamics of axially moving plates. J. Sound Vib. 2013, 332, 391-406. [CrossRef] 
42. Ghayesh, M.H.; Farokhi, H. Nonlinear dynamics of microplates. Int. J. Eng. Sci. 2015, 86, 60-73. [CrossRef]

43. Amabili, M. Nonlinear vibrations of viscoelastic rectangular plates. J. Sound Vib. 2016, 362, $142-156$. [CrossRef]

44. Wiggins, S. Global Bifurcations and Chaos: Analytical Methods; Springer Science \& Business Media: Heidenburg, Germany, 2013; Volume 73.

45. Feng, Z.; Wiggins, S. On the existence of chaos in a class of two-degree-of-freedom, damped, strongly parametrically forced mechanical systems with brokenO (2) symmetry. Zeitschrift für angewandte Mathematik und Physik ZAMP 1993, 44, 201-248. [CrossRef]

46. Zhang, W. Global and chaotic dynamics for a parametrically excited thin plate. J. Sound Vib. 2001, 239, 1013-1036. [CrossRef]

47. Hsu, C. A theory of cell-to-cell mapping dynamical systems. J. Appl. Mech. 1980, 47, 931-939. [CrossRef]

48. Xu, W.; He, Q.; Fang, T.; Rong, H. Stochastic bifurcation in Duffing system subject to harmonic excitation and in presence of random noise. Int. J. Non-Linear Mech. 2004, 39, 1473-1479. [CrossRef]

49. Crauel, H.; Flandoli, F. Additive noise destroys a pitchfork bifurcation. J. Dyn. Differ. Equ. 1998, 10, $259-274$. [CrossRef]

50. Esteban, M.; Núñez, E.P.; Torres, F. Bifurcation Analysis of Hysteretic Systems with Saddle Dynamics. Appl. Math. Nonlinear Sci. 2016, 2, 449-464.

51. Arnold, L.; Bleckert, G.; Schenk-Hoppé, K.R. The Stochastic Brusselator: Parametric Noise Destroys Hoft Bifurcation. In Stochastic Dynamics; Springer: New York, NY, USA , 1999; pp. 71-92.

52. Wang, B. Existence, stability and bifurcation of random complete and periodic solutions of stochastic parabolic equations. Nonlinear Anal. Theory Methods Appl. 2014, 103, 9-25. [CrossRef]

53. Schenk-Hoppé, K.R. Random attractors-General properties, existence and applications to stochastic bifurcation theory. Discret. Contin. Dyn. Syst.-A 1998, 4, 99-130. [CrossRef]

54. Caraballo, T.; Langa, J.A.; Robinson, J.C. A stochastic pitchfork bifurcation in a reaction-diffusion equation. In Proceedings of the Royal Society of London A: Mathematical, Physical and Engineering Sciences; The Royal Society: London, UK , 2001; Volume 457, pp. 2041-2061.

55. Temam, R. Infinite-Dimensional Dynamical Systems in Mechanics And Physics; Springer: New York, NY, USA, 1997.

56. Keller, H.; Ochs, G. Numerical approximation of random attractors. In Stochastic Dynamics; Springer: New York, NY, USA, 1999; pp. 93-115.

57. Lions, J.L.; Magenes, E. Non-Homogeneous Boundary Value Problems and Applications; Springer Science \& Business Media: Paris, France, 2012; pp. 7-8, 112-131, 275-276.

58. Debussche, A.; Temam, R. Some new generalizations of inertial manifolds. Discret. Contin. Dyn. Syst.-A 1996, 2, 543-558.

59. Marion, M.; Temam, R. Nonlinear galerkin methods. SIAM J. Numerical Anal. 1989, 26, 1139-1157. [CrossRef]

60. Multiphysics, A. COMSOL Multiphysics 3.5 a Reference Manual, PDE Mode Equation Based Modeling; Multiphysics Ltd: Stohkholm, Sweden, 2008.

61. Kloeden, P.; Eckhard, P. Numerical Solution of Stochastic Differential Equations; Springer: Berlin, Germany, 1992.

62. Schmalfuss, B. Measure attractors and random attractors for stochastic partial differential equations. Stochastic Anal. Appl. 1999, 17, 1075-1101. [CrossRef]

63. Crauel, H. Random point attractors versus random set attractors. J. Lond. Math. Soc. 2001, 63, 413-427. [CrossRef]

64. Birnir, B. Basic Attractors and Control; Springer: New York, NY, USA, 2015.

65. Adams, R.A.; Fournier, J.J. Sobolev Spaces; Academic Press: Cambridge, MA, USA , 2003.

66. Zheng, S. Nonlinear Evolution Equations; CRC Press: Boca Raton , FL, USA, 2004.

67. Chepyzhov, V.; Vishik, M. A Hausdorff dimension estimate for kernel sections of non-autonomous evolution equations. Indiana Univ. Math. J. 1993, 42, 1057-1076. [CrossRef]

68. Crisfield, M. A fast incremental/iterative solution procedure that handles "snap-through". In Computational Methods in Nonlinear Structural and Solid Mechanics; Elsevier: New York, NY, USA , 1981; pp. 55-62.

(C) 2018 by the authors. Licensee MDPI, Basel, Switzerland. This article is an open access article distributed under the terms and conditions of the Creative Commons Attribution (CC BY) license (http:/ / creativecommons.org/licenses/by/4.0/). 